\title{
ROBUST DENSITY MODELLING USING THE STUDENT'S $T$-DISTRIBUTION FOR HUMAN ACTION RECOGNITION
}

\author{
Zia Moghaddam and Massimo Piccardi \\ Faculty of Engineering and Information Technology, University of Technology, Sydney (UTS), Australia \\ \{ziam, massimo\}@it.uts.edu.au
}

\begin{abstract}
The extraction of human features from videos is often inaccurate and prone to outliers. Such outliers can severely affect density modelling when the Gaussian distribution is used as the model since it is highly sensitive to outliers. The Gaussian distribution is also often used as base component of graphical models for recognising human actions in the videos (hidden Markov model and others) and the presence of outliers can significantly affect the recognition accuracy. In contrast, the Student's $t$-distribution is more robust to outliers and can be exploited to improve the recognition rate in the presence of abnormal data. In this paper, we present an HMM which uses mixtures of $t$-distributions as observation probabilities and show how experiments over two well-known datasets (Weizmann, MuHAVi) reported a remarkable improvement in classification accuracy.
\end{abstract}

Index Terms- Observation density modelling, Student's $t$-distribution, Gaussian mixture model, hidden Markov model, human action recognition.

\section{INTRODUCTION}

Human action recognition is a very active research area in computer vision with main applications to video surveillance, human-computer interaction and so on. The goal of automatic action recognition is the classification of a given image sequence as one of pre-defined action classes. It requires the classification of a set of measurements extracted from the video depicting the action. Various human action recognition approaches can be categorised in two main groups [1]: 1) direct classification of spatiotemporal features using either a discriminative classifier, e.g. the support vector machine (SVM), or the $k$-Nearest Neighbour $(k \mathrm{NN})$ classifier; 2$)$ using temporal state-space models for action classification, either by recognising the actions directly in the time domain, e.g. dynamic time warping (DTW); or exploiting graphical models, e.g. hidden Markov model (HMM) [2-4] and conditional random fields (CRFs). Sequential classifiers such as HMM can naturally classify sequences of arbitrary length and have shown good performance in adjusting to temporal variations in the duration of instances of a same action. Hence, in this paper we decided to exploit HMM for action classification.
Prior to action classification, the HMM parameters need to be estimated to fit the model to the training observation sequences. The distribution of continuous observations in each HMM state is usually modelled by a Gaussian mixture model (GMM). However, the sequential data modelling may encounter the presence of unusual data (outliers) that cannot be fit by the normal tails of GMM. The covariance of the normal Gaussian usually has to be stretched to cover the outliers and leads to improper mixture fitting. Another alternative is to have many Gaussian components in the mixture, but that would affect computational efficiency of model training significantly.

To tackle the outlier problem in density estimation, the Student's $t$-distribution - which has a longer tail compared to the Gaussian density - has been exploited in many papers in the last few years [5-7]. For example, Chatzis et al. in [6] exploited the Student's $t$-mixture model (SMM) for sequential observation modelling of HMM states' densities to recognise the gestures of the American Sign Language. The obtained recognition error rate using SMM is $5.96 \%$ compared to $11.44 \%$ with GMM. For this reason, in this paper we apply a similar model to the problem of human action recognition.

The rest of the paper is organized as follows. Section 2 offers a review of the Student's $t$-distribution and its mixture (SMM). In Section 3, SMM is utilised as observation probability density in HMM, and the expectationmaximisation (EM) algorithm is exploited for maximum likelihood estimation (MLE) of its parameters. The experiments to explore the effectiveness of action classification using SMM density modelling in HMM are presented in Section 4. Finally, conclusions are given.

\section{THE STUDENT'S $t$-DISTRIBUTION}

The Student's $t$-distribution could be considered as an infinite mixture of scaled Gaussians with the same mean but variable variance (or covariance) produced by a prior Gamma distribution. In the case of an $F$-dimensional multivariate random variable $x$, with mean $\mu$ and inner product matrix $\psi$, this mixture can be written as [8]:

$$
\operatorname{St}(x \mid \mu, \psi, v)=\int_{0}^{\infty} \mathcal{N}\left(x \mid \mu, \frac{\psi}{\eta}\right) \operatorname{Gamma}\left(\eta \mid \frac{v}{2}, \frac{v}{2}\right) d \eta
$$




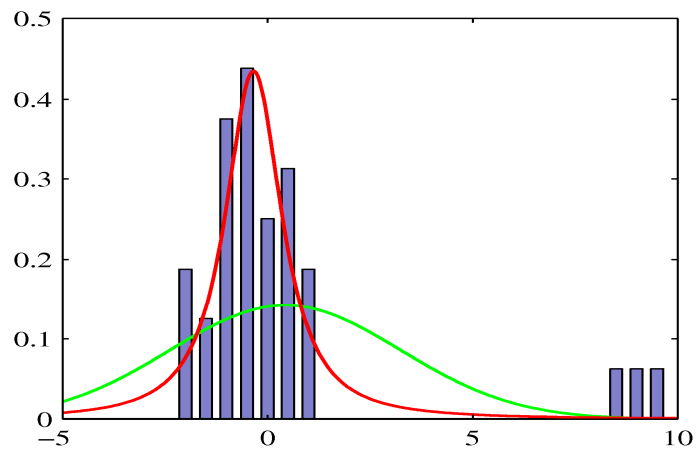

Fig. 1. Outliers impact on Gaussian compared to $t$-distribution [8].

The variable precision $\eta$ enables the $t$-distribution to give less weight to abnormal observations in the mixture parameter calculation which makes it more robust to outliers. Fig. 1 shows how the presence of the outliers can stretch the Gaussian distribution (green curve) and deform it, while the $t$-distribution (red curve) is influenced negligibly. The tail of the $t$-distribution is controlled by parameter $v$ (degrees of freedom); larger values of $v$ make the tail shorter and with $v \rightarrow \infty$ the $t$-distribution becomes a normal Gaussian distribution. The probability density function (pdf) of the $t$-distribution is given by [8]:

$$
S t(x \mid \mu, \psi, v)=\frac{\Gamma\left(\frac{v}{2}+\frac{F}{2}\right)|\psi|^{-\frac{1}{2}}}{\Gamma\left(\frac{v}{2}\right)(\pi v)^{\frac{F}{2}}}\left[1+\frac{\delta(x, \mu ; \psi)}{v}\right]^{-\frac{v}{2}-\frac{F}{2}}
$$

where, $\delta$ is the Mahalanobis distance between $x$ and the mean, $\mu$, with respect to $\psi$.

Like GMM, a set of $E$ independent multivariate data $\mathrm{X}=\left\{x_{e}\right\}_{e=1}^{E}$ could be generated by a mixture of $M$ Student's $t$-distribution components (SMM) [5]. Peel and McLachlan in [5] exploited the EM algorithm to estimate parameters of an SMM by derivation of the model's complete data loglikelihood function.

\section{THE HIDDEN MARKOV MODEL (HMM) WITH SMM OBSERVATION DENSITY MODELLING}

With sequential data, e.g. a time-sequence data, the order of observations is important. Hence, to model the sequential data, a sequential probabilistic model, like HMM, is required. HMM is a temporal graphical model in which the modelled system has observed outputs (observations), and a set of hidden states [9]. In the case of continuous observation, the observation distribution for each state in HMM is usually modelled by a GMM (HMM-GMM). However, the SMM can also be utilised to model the HMM observation to tackle the outlier issue [6]. Let us assume that observation sequence $O=\left\{o_{1}, \ldots, o_{t}, \ldots, o_{T}\right\}$ of length $T$ is generated by an $N$-state HMM. Furthermore, let us assume [10] that the observation probability for each state $s_{i}, i=1 \ldots N$, is modelled by an $M$-component SMM (HMM-SMM) as follows. For $i=1 \ldots N$ and $t=1 \ldots T$ :

$$
b_{i}\left(o_{t}\right)=p\left(o_{t} \mid q_{t}=s_{i}\right)=\sum_{l=1}^{M} c_{i l} \operatorname{St}\left(o_{t} \mid \mu_{i l}, \psi_{i l}, v_{i l}\right)
$$

where $\left\{c_{i l}\right\}_{l=1}^{M}$ are the mixing weights for the SMM of state $s_{i}$ such that $\sum_{l=1}^{M} c_{i l}=1 . \mu_{i l}, \psi_{i l}$ and $v_{i l}$ are the parameters of the $l^{\text {th }}$ component of SMM for state $s_{i}$.

\subsection{ML parameter estimation of an HMM-SMM}

Recalling from the EM algorithm for HMM-GMM [11], sequential data $O=\left\{o_{1}, \ldots, o_{t}, \ldots, o_{T}\right\}$ is considered incomplete and for each given $o_{t}$, it is assumed that two unobserved data exist whose values indicate the state value and the component index. In addition, similar to a single $t$ distribution and an SMM [5, 12], the precision scale $\eta$ is the third hidden variable in an HMM-SMM. As first step, we need to infer the conditional state posterior $\gamma_{i}(t)$, and the conditional component posterior $\tau_{i l}(t)$ defined as follows. For $i=1 \ldots N, l=1 \ldots M$ and $t=1 \ldots T$ :

$$
\begin{gathered}
\gamma_{i}(t)=p\left(q_{t}=s_{i} \mid O, \lambda\right) \\
\tau_{i l}(t)=p\left(z_{t i}=l \mid q_{t}=s_{i}, o_{t}, \lambda\right)
\end{gathered}
$$

Furthermore, we define $u_{i l}(t)$ as the conditional posterior expectation of precision scale for sample $o_{t}$ given that it is generated by the $l^{\text {th }}$ component of the $i^{\text {th }}$ mixture:

$$
u_{i l}(t)=E\left(\eta_{t} \mid z_{t i}=l, o_{t}, \lambda\right)
$$

At iteration $(k+1)$ of the EM algorithm:

E-step: The HMM observation density has no effect on the probability $\gamma_{i}(t)$, hence, it is same as the case of an HMMGMM [11]. The probability $\tau_{i l}(t)$ is also similar to that of HMM-GMM, except replacing the Gaussian by the $t$ distribution. The solution for $u_{i l}(t)$ is similar to that provided in [5] for a single SMM and just needs adding the index for the $i^{\text {th }}$ mixture.

M-step: The estimation formulas for HMM-SMM can be inferred by considering the influence of $u_{e l}$ (the conditional posterior expectation of precision scale) in the EM solution for SMM in [5] and comparing it to the EM solution for GMM in [11]. From [5], we can see that the mixture weight $c_{l}$ has the same formula as the GMM case. However, in SMM the posterior probability of precision scale $\eta$ will appear in the numerator and denominator of mean $\mu_{l}$, and in the numerator of precision $\psi_{l}$ (but not in its denominator). Hence, we can infer the following estimation formulas for HMM-SMM by putting together the corresponding formulas from [5] and [11]:

$$
c_{i l}^{(k+1)}=\frac{\sum_{t=1}^{T} \gamma_{i}(t) \tau_{i l}(t)}{\sum_{t=1}^{T} \gamma_{i}(t)}
$$




$$
\begin{gathered}
\mu_{i l}^{(k+1)}=\frac{\sum_{t=1}^{T} \gamma_{i}(t) \tau_{i l}(t) u_{i l}(t) o_{t}}{\sum_{t=1}^{T} \gamma_{i}(t) \tau_{i l}(t) u_{i l}(t)} \\
\psi_{i l}^{(k+1)}=\frac{\sum_{t=1}^{T} \gamma_{i}(t) \tau_{i l}(t) u_{i l}(t)\left(o_{t}-\mu_{i l}^{(k+1)}\right)\left(o_{t}-\mu_{i l}^{(k+1)}\right)^{T}}{\sum_{t=1}^{T} \gamma_{i}(t) \tau_{i l}(t)}
\end{gathered}
$$

Chatzis et al. in [6] obtained the same formulas by derivation of the complete data log-likelihood function. Nevertheless, similar to the single component $t$-distribution and SMM cases, $v_{i l}^{(k+1)}$ does not have a closed form solution, and it requires to be computed iteratively through the following equation, where $\Phi(x)$ is the digamma function:

$$
\begin{aligned}
& 1-\Phi\left(\frac{v_{i l}}{2}\right)+\ln \left(\frac{v_{i l}}{2}\right)+\Phi\left(\frac{v_{i l}^{(k)}+F}{2}\right)-\ln \left(\frac{v_{i l}^{(k)}+F}{2}\right) \\
& +\frac{\sum_{e=1}^{E} \sum_{t=1}^{T_{e}} \gamma_{i}^{e}(t) \tau_{i l}^{e}(t)\left[\ln \left(u_{i l}^{e}(t)\right)-u_{i l}^{e}(t)\right]}{\sum_{e=1}^{E} \sum_{t=1}^{T_{e}} \gamma_{i}^{e}(t) \tau_{i l}^{e}(t)}=0
\end{aligned}
$$

\section{EXPERIMENTS ON ROBUSTNESS USING THE $\boldsymbol{t}$-DISTRIBUTION}

In this section, we explore the robustness of action recognition by exploiting HMM-SMM in lieu of HMMGMM. For this purpose, we modified Kevin Murphy's HMM toolbox [13] to include HMM with SMM density modelling.

For the experiment, we exploited two action video datasets: 1) the popular Weizmann dataset [14] that includes 10 actions performed by 9 subjects for a total of 93 videos sequences; 2) the more recent MuHAVi dataset [10] that contains videos from 17 action classes performed several times by 7 different subjects and captured by 8 cameras simultaneously. To the best of our knowledge, MuHAVi is the most challenging and comprehensive public action dataset to date in terms of combined number of action classes, subjects and cameras. Amongst the other popular datasets, KTH [15] has only 6 action classes.

In order to separate issues raised by recognition and foreground segmentation, the authors of the MuHAVi dataset aimed to make manually annotated silhouettes available (MuHAVi-MAS). However, manual segmentation proved overly time consuming and the current number of manually-segmented sequences is rather limited. Furthermore, using manually-generated masks for training or validation would provide an optimistic estimate of a method's accuracy compared to a real application where segmentation must be automated. Hence, we decided to use the original videos directly and extract the object masks automatically from the camera 4 viewpoint, resulting in 398 sample sequences [16]. The quality of the obtained masks is rather good, yet not as that of manual segmentation, making results more significant and general.

As validation approach, we have used the "leave-onesubject-out" cross validation method; i.e. in each run we leave one subject out during training and we use it for testing. This validation procedure is realistic since in real applications subjects would not have been seen during training. The final accuracy result is the average over the various subjects ( 7 folds). Since the number of HMM states, $N$, and the number of components per state, $M$, are hyperparameters in the Baum-Welch algorithm and cannot be determined by maximum likelihood, we decided to experiment over range $\{1 \ldots 6\}$ for $N$ and $M$, and choose the best combination based on cross-validation accuracy.

Another choice required to complete the recognition approach is that of the feature set. Given the tight real-time constraints of video surveillance, for our experiments we have chosen to compare two simple and computationally lightweight feature sets: the first is the well-known projection histograms [17] (our implementation is in [16]) and the second is our own "sectorial extreme points" feature set, explained in details in [4].

\subsection{Experiments with HMM-SMM and estimated $v$}

In the first experiment, we compared the classification accuracy between HMM-SMM density modelling with maximum likelihood-estimated $v$ and HMM-GMM. For this experiment, parameter $v$ is initialised with an arbitrary value (10 in the experiment), and is updated at each M-step iteration. To prevent $v$ from tending towards 0 or infinite on occasions, we also placed a minimum and a maximum threshold for the updated values of $v: 0.1$ and 200, respectively. Table 1 reports the average and best classification accuracies obtained over 6 different runs from random initial parameters for both HMM-GMM and HMMSMM with estimated $v$ using MuHAVi as dataset and the projection histograms as feature set. The achieved results show considerable improvements in accuracy when using HMM-SMM for all combinations of $N$ and $M$. The increase in highest accuracy is over 6\% (from $80.7 \%$ to $86.9 \%$ ). It seems that the main reason for this improvement is the significant presence of outliers in the observation data using this feature set on the MuHAVi dataset.

We repeated the experiment with the other feature set, the sectorial extreme points, on both the Weizmann and MuHAVi datasets. Table 2 summarises the average and best accuracies over the 6 runs for various values of $N$ and $M$. While the achieved improvement over MuHAVi was limited with this feature set (likely because of the lack of significant outliers), over the Weizmann dataset HMM-SMM reached the maximum possible accuracy of $100 \%$ (not one single misclassification).

Given the apparent sensitivity of the ML-estimate of $v$, we compared accuracy also with fixing $v$ to an arbitrary value ( 0.1 in this case); the feature set is the projection histograms and the dataset is MuHAVi, with results reported in Table 3. Comparison of results in Tables 1 and 3 shows that the classification accuracy using HMM-SMM with fixed $v=0.1$ was comparable with that of HMM-SMM with estimated $v$. 
Table 1: Classification accuracy (\%) for HMM-GMM and HMMSMM with estimated $v$ using the projection histograms feature set.

HMM-GMM, Avr. of 6 runs HMM-SMM, Avr. of 6 runs \begin{tabular}{ll}
$\mathrm{M}=1|\mathrm{M}=2 \mathrm{M}=3| \mathrm{M}=4|\mathrm{M}=5| \mathrm{M}=6$ \\
$\mathrm{M}=1|\mathrm{M}=2| \mathrm{M}=3|\mathrm{M}=4| \mathrm{M}=5 \mid \mathrm{M}=6$ \\
\hline
\end{tabular} \begin{tabular}{|l|l|l|l|l|l|l|l|l|l|l|l|l|l|}
\hline $\mathrm{N}=1$ & 64.1 & 70.4 & 73.5 & 74.2 & 74.3 & 74.5 & 83.7 & 85 & 84.1 & 84.5 & 84.5 & $\mathbf{8 5 . 1}$ \\
\hline
\end{tabular} \begin{tabular}{|l|l|l|l|l|l|l|l|l|l|l|l|l|l|l|l|}
\hline $\mathrm{N}=2$ & 67.6 & 71.8 & 72.4 & 72.2 & 72.2 & 72.7 & 84.7 & 84.8 & 83.9 & 84.6 & 84.4 & 83.7 \\
\hline
\end{tabular}

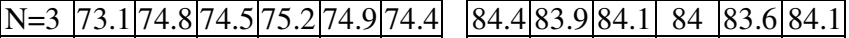

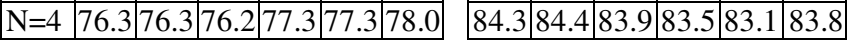
\begin{tabular}{|l|l|l|l|l|l|l|l|l|l|l|l|l|l|}
\hline $\mathrm{N}=5$ & 76.4 & 78.5 & 78.1 & 77.3 & 77.8 & 77.8 & 84.5 & 84.2 & 83.5 & 84.0 & 84.0 & 83.3 \\
\hline
\end{tabular} \begin{tabular}{|l|l|l|l|l|l|l|l|l|l|l|l|l|l|l|}
$\mathrm{N}=$ & & &
\end{tabular}

HMM-GMM, Best of 6 runs HMM-SMM, Best of 6 runs

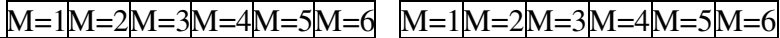

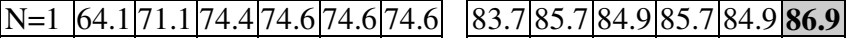
\begin{tabular}{|l|l|l|l|l|l|l|l|l|l|l|l|l|l|}
\hline $\mathrm{N}=2$ & 69.1 & 73.1 & 74.1 & 73.9 & 73.9 & 74.4 & 83.7 & 85.7 & 86.2 & 84.4 & 86.2 & 85.4 & 84.9 \\
\hline
\end{tabular} \begin{tabular}{|l|l|l|l|l|l|l|l|l|l|l|l|l|l|}
\cline { 1 - 7 } & $\mathrm{N}=3$ & 74.4 & 79.6 & 76.6 & 76.6 & 76.4 & 75.6 & 84.9 & 85.9 & 84.9 & 85.4 & 84.4 & 85.4 \\
\hline
\end{tabular}

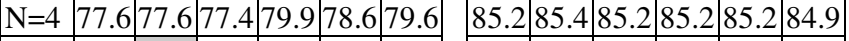
\begin{tabular}{|l|l|l|l|l|l|l|l|l|l|l|l|l|l|l|l|l|}
\cline { 1 - 6 } & $\mathbf{N}=5$ & 77.9 & $\mathbf{8 0 . 7}$ & 80.2 & 79.4 & 79.1 & 79.1 & 85.2 & 85.4 & 84.2 & 85.2 & 84.9 & 85.4 \\
\hline
\end{tabular}

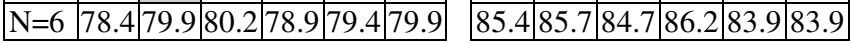

Table 2: Classification accuracy (\%) for HMM-GMM and HMMSMM with estimated $v$ using the sectorial extreme points feature set.

\begin{tabular}{|c|c|c|c|c|}
\hline \multirow{2}{*}{ Dataset } & \multicolumn{2}{|c|}{ HMM-GMM } & \multicolumn{2}{c|}{ HMM-SMM } \\
\cline { 2 - 5 } & $\begin{array}{c}\text { Average of } \\
\text { 6 runs }\end{array}$ & $\begin{array}{c}\text { Best of 6 } \\
\text { runs }\end{array}$ & $\begin{array}{c}\text { Average of } \\
\text { 6 runs }\end{array}$ & $\begin{array}{c}\text { Best of 6 } \\
\text { runs }\end{array}$ \\
\hline Weizmann & 94.6 & 96.8 & 97.6 & 100 \\
\hline MuHAVi & 92.4 & 94.0 & 93.0 & 94.5 \\
\hline
\end{tabular}

Table 3: Classification accuracy (\%) for HMM-SMM with estimated $v$ and fixed $v$ using the projection histograms feature set. \begin{tabular}{|l|l|}
\hline HMM-SMM with estimated $\boldsymbol{v}$ & HMM-SMM with fixed $\boldsymbol{v}=0.1$ \\
\hline
\end{tabular}

\begin{tabular}{|c|c|c|c|}
\hline Avr. of 6 runs & Best of 6 runs & Avr. of 6 runs & Best of 6 runs \\
\hline 85.1 & 86.9 & 85.3 & 86.4 \\
\hline
\end{tabular}

Table 4: Classification accuracy (\%) for HMM-SMM with various fixed $v$ using the projection histograms feature set.

\begin{tabular}{|c|c|c|c|c|}
\hline & $v=3$ & $v=5$ & $v=7$ & $v=10$ \\
\hline Average of 6 runs & 86.2 & 85.5 & 84.9 & 83.5 \\
\hline Best of 6 runs & 87.7 & 86.9 & 86.9 & 85.2 \\
\hline
\end{tabular}

\subsection{Experiments with HMM-SMM and various fixed values of $v$}

To confirm the usability of a fixed $v$, we measured the recognition accuracy with various fixed values of $v=\{3,5$, 7, 10\}; results are shown in Table 4. Such results confirm the effectiveness of SMM density modelling with fixed $v$ since maximum accuracy reaches $87.7 \%$ (with $v=3$ ). A relatively small search over values of $v$ is therefore capable of delivering cross-validation accuracies greater than that of the ML-estimated case $(86.9 \%)$. Therefore, estimation of $v$ could be avoided altogether in applications.

\section{CONCLUSIONS}

The longer tails in the Student's $t$-distribution (compared to the Gaussian distribution) makes density modelling more robust in the presence of outliers in the observation data. In this paper, we have utilised a mixture of $t$-distributions (SMM) to model the observation probabilities in an HMM in lieu of the usual GMM for application to human action recognition in videos. The EM algorithm was exploited to estimate the HMM-SMM parameters with a maximum likelihood objective. The experiments conducted over two significant video action datasets have proved the effectiveness of using HMM-SMM, with accuracy improvements in the order of $6 \div 7 \%$ over the conventional Gaussian mixture models.

\section{REFERENCES}

[1] R. Poppe, "A Survey on Vision-Based Human Action Recognition," IVC, vol. 28, no. 6, 2010, pp. 976-990.

[2] J. Yamato, J. Ohya and K. Ishii, "Recognizing Human Action in Time-Sequential Images using Hidden Markov Model," in CVPR 1992, pp. 379-385.

[3] F. Martinez-Contreras, C. Orrite-Urunuela, E. HerreroJaraba, H. Ragheb and S.A. Velastin, "Recognizing Human Actions using Silhouette-Based HMM," in AVSS 2009, pp. 43-48.

[4] Z. Moghaddam and M. Piccardi, "Deterministic Initialization of Hidden Markov Models for Human Action Recognition," in DICTA 2009, pp. 188-195.

[5] D. Peel and G.J. McLachlan, "Robust Mixture Modelling using the t Distribution," Statistics and Computing, vol. 10, no. 4, 2000, pp. 339-348.

[6] S.P. Chatzis, D.I. Kosmopoulos and T.A. Varvarigou, "Robust Sequential Data Modeling Using an Outlier Tolerant Hidden Markov Model," TPAMI, vol. 31, no. 9, 2009, pp. 16571669.

[7] O.P. Concha, R. Xu and M. Piccardi, "Robust Dimensionality Reduction for Human Action Recognition," in DICTA 2010, pp. 349-356.

[8] C.M. Bishop, "Pattern Recognition and Machine Learning", Springer, 2006.

[9] L.R. Rabiner, "A Tutorial on Hidden Markov Models and Selected Applications in Speech Recognition," Proceedings of the IEEE, vol. 77, no. 2, 1989, pp. 257-286.

[10] Kingston University, "The Muhavi-MAS Database," http://dipersec.king.ac.uk/MuHAVi-MAS/, 2008 (last updated on 21 Jan. 2009).

[11] J.A. Bilmes, "A Gentle Tutorial of the EM Algorithm and its Application to Parameter Estimation for Gaussian Mixture and Hidden Markov Models," Technical Report ICSI-TR-97-01, University of California Berkeley 1998.

[12] C. Liu and D.B. Rubin, "ML Estimation of the t Distribution using EM and its Extensions, ECM and ECME," Statistica Sinica, vol. 5, no. 1, 1995, pp. 19-39.

[13] Kevin Murphy, "Hidden Markov Model Toolbox for Matlab," http://www.cs.ubc.ca/ murphyk/Software/HMM/hmm.html, 1998 (last updated on 8 Jun. 2005).

[14] M. Blank, L. Gorelick, E. Shechtman, M. Irani and R. Basri, "Actions as Space-Time Shapes," in ICCV 2005, pp. 1395-1402.

[15] C. Schuldt, I. Laptev and B. Caputo, "Recognizing Human Actions: A Local SVM Approach," in ICPR 2004, pp. 32-36.

[16] Z. Moghaddam and M. Piccardi, "Histogram-Based Training Initialisation of Hidden Markov Models for Human Action Recognition,” in AVSS 2010, pp. 256-261.

[17] R. Vezzani, D. Baltieri and R. Cucchiara, "HMM based action recognition with projection histogram features," in Proc.of the 20th Intl. Conf. on Recognizing patterns in signals, speech, images, and videos, Springer-Verlag, 2010, pp. 286-293. 


\section{IEEE $X$ plore}

Browse $>$ Conferences $>$ Image Processing (ICIP), 2011

Robust density modelling using the student's t-distribution for human action recognition

Moghaddam, Z. ; Piccardi, M. ;

Fac. of Eng. \& Inf. Technol., Univ. of Technol., Sydney, NSW, Australia

This paper appears in: Image Processing (ICIP), 2011 18th IEEE International Conference on

I ssue Date : 11-14 Sept. 2011

On page(s): $3261-3264$

ISSN : $1522-4880$

E-ISBN : 978-1-4577-1302-6

Print I SBN: 978-1-4577-1304-0

I NSPEC Accession Number: 12505028

Digital Object I dentifier : $10.1109 /$ ICIP.2011.6116366

Date of Current Version : 29 December 2011

\section{ABSTRACT}

The extraction of human features from videos is often inaccurate and prone to outliers. Such outliers can severely affect density modelling when the Gaussian distribution is used as the model since it is highly sensitive to outliers. The Gaussian distribution is also often used as base component of graphical models for recognising human actions in the videos (hidden Markov model and others) and the presence of outliers can significantly affect the recognition accuracy. In contrast, the Student's t-distribution is more robust to outliers and can be exploited to improve the recognition rate in the presence of abnormal data. In this paper, we present an HMM which uses mixtures of t-distributions as observation probabilities and show how experiments over two well-known datasets (Weizmann, MuHAVi) reported a remarkable improvement in classification accuracy.

\section{INDEX TERMS}

- IEEE Terms

Accuracy, Data models, Hidden Markov models, Histograms, Humans, Robustness, Videos

- INSPEC

Controlled I ndexing

Gaussian distribution, feature extraction, hidden Markov models, image recognition, video signal processing

Non Controlled I ndexing

Gaussian distribution, HMM , hidden Markov models, human action recognition, human feature extraction, robust density modelling , student tdistribution, videos

- Author Keywords

Gaussian mixture model, Observation density modelling , Student's t-distribution, hidden Markov model, human action recognition 


\section{MA.L1: Modern Shape from Shading and Beyond (Special Session)}

\section{Modern Shape From Shading and Beyond}

Michael Breuss (Saarland University, Germany); Oliver Vogel (Saarland University, Germany); Ariel Tankus (Technion - Israel Institute of Technology, Israel) pp. 1-4

Numerical Schemes for Advanced Reflectance Models for Shape From Shading Oliver Vogel (Saarland University, Germany); Emiliano Cristiani (SAPIENZA Universita di Roma, Italy) pp. 5-8

Break

Shape From Shading with Specular Highlights: Analysis of the Phong Model Michael Breuss (Saarland University, Germany); Yong Chul Ju (Saarland University, Germany) pp. 9-12

\section{Shape-from-shading Under Complex Natural Illumination}

Rui Huang (University of York, United Kingdom); William Smith (University of York, United Kingdom)

pp. 13-16

Reconstruction of non-Lambertian Surfaces by Fusion of Shape From Shading and Active Range Scanning

Steffen Herbort (Dortmund University of Technology, Germany); Arne Grumpe (Dortmund University of Technology, Germany); Christian Wöhler (Dortmund University of Technology, Germany) pp. $17-20$

Shape From Specular Reflection in Calibrated Environments and the Integration of Spatial Normal Fields Jonathan Balzer (King Abdullah University of Science and Technology, Saudi Arabia) pp. 21-24

\section{MA.L2: Structural Models (Lecture)}

Joint Pose Estimation and Action Recognition in Image Graphs

Raja Kumar (IRISA \& INRIA Rennes, France); Ivan Laptev (INRIA Paris -

Rocquencourt, France); Patrick Pérez (INRIA, France); Lionel Oisel (Thomson,

France)

pp. 25-28

\section{Inferring 3D Body Pose Using Variational Semi-parametric Regression}

Yan Tian (Hikvision Digital Technology Co. Ltd, P.R. China); Yonghua Jia (Hikvision Digital Technology Co. Ltd, P.R. China); Yuan Shi (Carnegie Mellon University, USA); 
Yong Liu (Beijing University of Posts and Telecommunications, P.R. China); Ji Hao (Carnegie Mellon University, USA); Leonid Sigal (Disney Research Pittsburgh, USA) pp. 29-32

Break

Automatic Target Recognition Using Discriminative Graphical Models

Umamahesh Srinivas (Pennsylvania State University, USA); Vishal Monga

(Pennsylvania State University, USA); Raghu Raj (None, USA)

pp. 33-36

A Belief Propagation Algorithm for Bias Field Estimation and Image Segmentation

Rui Huang (Huazhong University of Science and Technology, P.R. China); Nong Sang (Huazhong University of Science and Technology, P.R. China); Vladimir Pavlovic

(Rutgers, USA); Dimitri Metaxas (Rutgers University, USA)

pp. $37-40$

Planarity-Enforcing Higher-Order Graph Cut

Thomas Weibel (Fraunhofer ITWM \& CRAN-ENSEM-INPL, Germany); Christian Daul (CRAN INPL Nancy, France); Didier Wolf (CRAN INPL Nancy, France); Ronald Rösch (Fraunhofer ITWM, Germany)

pp. 41-44

Learning Structural Conjunction of Image Content by Sparse Graphical Model Donghui Wang (Zhejiang University, P.R. China); Xiao Deng (Zhejiang University, P.R. China) pp. 45-48

\section{MA.L3: Biomedical Image Analysis (Lecture)}

Automatic Quality Enhancement and Nerve Fibre Layer Artefacts Removal in Retina Fundus Images by Off Axis Imaging

Luca Giancardo (Oak Ridge National Laboratory \& University of Burgundy, USA);

Fabrice Meriaudeau (Uni Bourgogne, France); Thomas Karnowski (Oak Ridge

National Laboratory, USA); Yaqin Li (U. Tennessee Health Sciences Center, USA);

Kenneth W. Tobin (Oak Ridge National Laboratory, USA); Edward Chaum (U.

Tennessee Health Sciences Center, USA)

pp. $49-52$

Advanced Statistical Matrices for Texture Characterization: Application to DNA Chromatin and Microtubule Network Classification

Guillaume Thibault (Centre de Morphologie Mathematique \& MINES PARISTECH,

France); Jesus Angulo (MINES Paristech, France); Fernand Meyer (Mines ParisTech, Ecole des Mines de Paris, France)

pp. 53-56

Break

Comparison of Energy Minimization Methods for 3-D Brain Tissue Classification

Subrahmanyam Gorthi (EPFL \& LTS5, Switzerland); Jean-Philippe Thiran (École

Polytechnique Fédérale de Lausanne \& Signal Processing Laboratory, Switzerland);

Meritxell Bach Cuadra (Ecole Polytechnique Fédérale de Lausanne (EPFL),

Switzerland) 
pp. $57-60$

\section{Clump Splitting Via Bottleneck Detection}

Maddy Hui Wang (University of Alberta, Canada); Hong Zhang (University of Alberta, Canada); Nilanjan Ray (University of Alberta, Canada)

pp. 61-64

Texture Classification of Scarred and Non-Scarred Myocardium in Cardiac MRI Using Learned Dictionaries

Lasya Kotu (University of Stavanger, Norway); Kjersti Engan (University of Stavanger, Norway); Trygve Eftestøl (University of Stavanger, Norway); Stein Ørn (Stavanger University Hospital, Norway); Leik Woie (Stavanger University Hospital, Norway) pp. 65-68

\section{Automatic IVUS Media-Adventitia Border Extraction Using Double Interface Graph Cut Segmentation \\ Ehab Essa (Swansea University, United Kingdom); Xianghua Xie (Swansea University, United Kingdom); Igor Sazonov (Swansea, United Kingdom); Perumal Nithiarasu (Swansea University, United Kingdom) \\ pp. 69-72}

\section{MA.L4: Nonlinear and Linear Filtering (Lecture)}

Non-Linearization of Free Schrödinger Equation and Pseudo-Morphological Complex Diffusion Operators Jesus Angulo (MINES Paristech, France) pp. 73-76

Adaptive Filtering of Raster Map Images Using Optimal Context Selection Minjie Chen (University of Eastern Finland, Finland); Mantao Xu (Shanghai Dian Ji University, P.R. China); Pasi Fränti (University of Eastern Finland, Finland) pp. $77-80$

Break

FM Processing with Generalized Amplitude \& Phase: Application to Modulation Domain Geometric Image Transformations

Chuong Nguyen (University of Oklahoma, USA); Jonathan Williams (University of Oklahoma, USA); Joseph P. Havlicek (University of Oklahoma, USA); Murad Özaydin (University of Oklahoma, USA) pp. 81-84

\section{Approximating Image Filters with Box Filters}

Bernardo Pires (Carnegie Mellon University, USA); Karanhaar Singh (Carnegie Mellon University, USA); Jose Moura (Carnegie Mellon University, USA) pp. 85-88

Cosine Integral Images for Fast Spatial and Range Filtering Elhanan Elboher (The Hebrew University of Jerusalem, Israel); Michael Werman (Hebrew University of Jerusalem, Israel) pp. 89-92 


\section{An Optimal Design of FIR Filters with Discrete Coefficients and Image Sampling}

Application

Ha H Kha (University of Technology Sydney, Australia); Hoang D. Tuan (University of Technology, Sydney, Australia); Truong Nguyen (University of California in San Diego, USA) pp. 93-96

\section{MA.L5: Image Search and Retrieval (Lecture)}

\section{Exploiting Contextual Information for Rank Aggregation}

Daniel Pedronette (University of Campinas, Brazil); Ricardo da Silva Torres (Institute of Computing, State University of Campinas, Brazil) pp. $97-100$

\section{Local Geometric Consistency Constraint for Image Retrieval} Hongtao Xie (Institute of Computing Technology, Chinese Academy of Sciences, P.R. China); Ke Gao (Institute of Computing Technology, Chinese Academy of Sciences, P.R. China); Yongdong Zhang (Institute of Computing Technology, Chinese Academy of Sciences, P.R. China); Jintao Li (Institute of Computing Technology, Chinese Academy of Sciences, P.R. China)

pp. 101-104

Break

Generating Vocabulary for Global Feature Representation Towards Commerce Image Retrieval

Zhang Chen (Peking University, P.R. China); Lingyu Duan (Peking University, P.R. China); Wang Chunyu (Peking University, P.R. China); Tiejun Huang (Peking University, P.R. China); Wen Gao (Peking University, P.R. China) pp. $105-108$

\section{Efficient Bag-of-Feature Kernel Representation for Image Similarity Search} Frederic Precioso (LIP6 CNRS UMR 7606 \& UPMC Paris 6, France); Matthieu Cord (UPMC Paris 6, France); David Gorisse (ETIS, CNRS, ENSEA, UniversityCergyPontoise, France); Nicolas Thome (University Pierre et Marie Curie, France) pp. 109-112

\section{Grid-based Local Feature Bundling for Efficient Object Search and Localization} Jiang Yuning (Nanyang Technology University, Singapore); Jingjing Meng (Motorola Inc., USA); Junsong Yuan (Nanyang Technological University, Singapore) pp. $113-116$

\section{MA.L6: 3D Coding and Representation (Lecture)}

\section{Block-Adaptive Palette-based Prediction for Depth Map Coding}

Shinya Shimizu (NTT Corporation, Japan); Hideaki Kimata (NTT Corporation, Japan); Shiori Sugimoto (NTT Corporation, Japan); Norihiko Matsuura (NTT Corporation, Japan) pp. $117-120$ 
Region-based Adaptive Bilateral Filter in Depth Map Coding

Ilsoon Lim (Samsung Electronics, Korea); Hochen Wey (Samsun Advanced Institute of Technology, Samsung Electronics Co., Ltd., Korea); Jaejoon Lee (Samsung Electronics, Korea)

pp. 121-124

Break

Object-based Layered Depth Images for Improved Virtual View Synthesis in RateConstrained Context

Vincent Jantet (ENS Cachan, Antenne de Bretagne, France); Christine Guillemot (INRIA, France); Luce Morin (INSA-Rennes, France) pp. $125-128$

Transform Domain Sparsification of Depth Maps Using Iterative Quadratic Programming Gene Cheung (National Institute of Informatics, Japan); Junichi Ishida (Chuo University, Japan); Akira Kubota (Chuo University, Japan); Antonio Ortega (USC, USA)

pp. 129-132

Sparse Stereo Image Coding with Learned Dictionaries

Dimitri Palaz (EPFL, Switzerland); Ivana Tošić (UC Berkeley, USA); Pascal Frossard (Swiss Federal Institute of Technology - EPFL, Switzerland) pp. 133-136

Efficient Compression Method for Integral Images Using Multi-view Video Coding Shasha Shi (Télécom-Bretagne \& France Télécom, France); Patrick Gioia (Orange Labs, France); Gérard Madec (Télécom-Bretagne, France) pp. $137-140$

\section{MA.L7: Error Resilience and Channel Coding for Image \& Video Systems (Lecture)}

Belief Consensus for Distributed Action Recognition

Ahmed T Kamal (University of California, Riverside, USA); Bi Song (University of California at Riverside, USA); Amit Roy-Chowdhury (University of California, Riverside, USA)

pp. $141-144$

Content-Aware Utility-Fair Video Streaming in Wireless Broadcasting Networks Wen Ji (Institute of Computing Technology, Chinese Academy of Sciences, P.R. China); Zhu Li (Huawei Technology USA, USA); Yiqiang Chen (Institute of Computing Technology, Chinese Academy of Sciences, P.R. China)

pp. $145-148$

Break

Resource Management for Wireless Visual Sensor Networks Based on Individual Video Characteristics

Angeliki Katsenou (University of loannina, Greece); Lisimachos P Kondi (University of Ioannina, Greece); Konstantinos Parsopoulos (University of loannina, Greece) 
pp. $149-152$

Tracking-Optimized Quantization for H.264 Compression in Transportation Video Surveillance Applications

Eren Soyak (Northwestern University, USA); Sotirios A. Tsaftaris (Northwestern University, USA); Aggelos K. Katsaggelos (Northwestern University, USA) pp. 153-156

\section{Dynamic GOP Size Control for Low-Delay Distributed Video Coding}

Krishna Rao Vijayanagar (Illinois Institute of Technology, USA); Joohee Kim (Illinois Institute of Technology, USA)

pp. $157-160$

\section{Optimal Image Transmission Over Visual Sensor Networks} sungjin Lee (Yonsei University, Korea); Sanghoon Lee (Yonsei University, Korea); Alan C Bovik (University of Texas at Austin, USA) pp. 161-164

\section{MA.L8: Radar Imaging and Remote Sensing (Lecture)}

\section{Bayesian TV Denoising of SAR Images}

Mlguel Vega (University of Granada, Spain); Javier Mateos (University of Granada, Spain); Rafael Molina (Universidad de Granada, Spain); Aggelos K. Katsaggelos (Northwestern University, USA) pp. $165-168$

SAR Image Classification with Non-stationary Multinomial Logistic Mixture of Amplitude and Texture Densities

Koray Kayabol (INRIA, France); Aurelie Voisin (INRIA, France); Josiane Zerubia (INRIA, Sophia Antipolis, France)

pp. 169-172

Break

Sparsity-driven Image Formation and Space-variant Focusing for SAR Özben Önhon (Sabanci University, Turkey); Müjdat Çetin (Sabanci University, Turkey) pp. $173-176$

Incremental Evolution of Collective Network of Binary Classifier for Polarimetric SAR Image Classification

Stefan Uhlmann (Tampere University of Technology, Finland); Serkan Kiranyaz (Tampere University of Technology, Finland); Moncef Gabbouj (Tampere University of Technology \& Tampere, Finland, Finland); Turker Ince (, Turkey) pp. $177-180$

Multispectral Interest Points for RGB-NIR Image Registration

Damien Firmenich (EPFL, Switzerland); Matthew Brown (EPFL, Switzerland); Sabine Süsstrunk (EPFL, Switzerland) pp. 181-184

\section{Contrast Enhancement and Denoising of Poisson and Gaussian Mixture Noise for}

Solar Images

Bojana Begovic (Royal College Building 204 George Street \& University of

Strathclyde, EEE Department, United Kingdom); Vladimir Stankovic (University of 
Strathclyde, United Kingdom); Lina Stankovic (University of Strathclyde, United Kingdom)

pp. $185-188$

MA.PA: Image \& Video Representation - Perception and Quality Models for Images \& Video (Poster)

An Incremental/Decremental Delaunay Mesh-Generation Framework for Image

Representation

Michael D. Adams (University of Victoria, Canada)

pp. 189-192

A Symmetric KL Divergence Based Spatiogram Similarity Measure

Zhijun Yao (Hua Zhong University of Science and Technology, P.R. China);

Zhongyuan Lai (Huazhong University of Science and Technology, P.R. China); Wenyu Liu (Huazhong University of Science and Technology, P.R. China)

pp. 193-196

Online Sparse Learning Utilizing Multi-Feature Combination for Image Classification Lihe Zhang (Dalian University of Technology, P.R. China)

pp. $197-200$

Evaluation Framework on Translation-Invariant Representation for Cumulative Foot

Pressure Image

Shuai Zheng (Institute of Automation, Chinese Academy of Sciences, P.R. China);

Kaiqi Huang (Chinese Academy of Sciences, P.R. China); Tieniu Tan (NLPR, P.R.

China)

pp. 201-204

Image Pattern Discovery by Using the Spatial Closeness of Visual Code Words Meng Sun (Katholieke Universiteit Leuven, Belgium); Hugo Van hamme (Universtity of Leuven, Belgium)

pp. 205-208

Action Recognition Using Correlogram of Body Poses and Spectral Regression Ling Shao (The University of Sheffield, United Kingdom); Di Wu (The University of Sheffield, United Kingdom); Xiuli Chen (The University of Manchester, United Kingdom)

pp. 209-212

\section{A Discriminative Learning Technique for Mobile Landmark Recognition}

Tao Chen (Nanyang Technological University, Singapore); Kim Hui Yap (Nanyang Technological University, Singapore); Lap-Pui Chau (Nanyang Technological University, Singapore)

pp. 213-216

SSIM-based Non-local Means Image Denoising

Abdul Rehman (University of Waterloo, Canada); Zhou Wang (University of Waterloo, Canada)

pp. $217-220$ 
Biologically Motivated Feature Extraction Using the Spiral Architecture

Bryan W. Scotney (University of Ulster, United Kingdom); Sonya A Coleman

(University of Ulster, United Kingdom); Bryan Gardiner (University of Ulster, United

Kingdom)

pp. 221-224

Accurate Distortion Measurement for B-Spline-Based Shape Coding

Zhongyuan Lai (Huazhong University of Science and Technology, P.R. China); Zhen

Zuo (Huazhong University of Science and Technology, P.R. China); Zhe Wang

(Huazhong University of Science and Technology, P.R. China); Zhijun Yao (Hua

Zhong University of Science and Technology, P.R. China); Wenyu Liu (Huazhong

University of Science and Technology, P.R. China)

pp. 225-228

\section{Abnormal Motion Selection in Crowds Using Bottom-Up Saliency}

Matei Mancas (University of Mons, Belgium); Nicolas Riche (University of Mons,

Belgium); Julien Leroy (University of Mons, Belgium); Bernard Gosselin (University of Mons, Belgium)

pp. 229-232

Motion Trajectory Based Visual Saliency for Video Quality Assessment

Lin Ma (Department of Electronic Engineering, The Chinese University of Hong Kong, Hong Kong); Songnan Li (The Chinese University of Hong Kong, Hong Kong); King N. Ngan (Chinese University of Hong Kong, Hong Kong)

pp. 233-236

Perceptual Noise Shaping in Dual-Tree Complex Wavelet Transform for Image Coding Junwu Zhu (Carleton University, Canada); Richard Dansereau (Carleton University, Canada); Chris Joslin (Carleton University, Canada) pp. 237-240

Fractal Image Coding Using SSIM Jianji Wang (Xi'an Jiaotong University, P.R. China); Yuehu Liu (Xi'an Jiaotong University, P.R. China); Ping Wei (Xi'an Jiaotong University, P.R. China); Zhiqiang Tian (Xi'an Jiaotong University, P.R. China); Yaochen Li (Xi'an Jiaotong University, P.R. China); Nanning Zheng (Xi'an Jiaotong University, P.R. China) pp. 241-244

\section{MA.PB: Multi-Resolution Methods for Image Processing (Poster)}

\section{Block-Lifting Factorization of M-Channel Biorthogonal Filter Banks with an Arbitrary McMillan Degree}

Taizo Suzuki (College of Engineering, Nihon University, Japan)

pp. $245-248$

Multiscale Directional AM-FM Demodulation of Images Using a 2D Optimized Method Victor Murray (University of New Mexico, USA); Marios Pattichis (University of New Mexico, USA); Peter Soliz (Vision Quest Biomedical, USA) pp. $249-252$

Design of Q-Shift Filters with Improved Vanishing Moments for DTCWT Xi Zhang (The University of Electro-Communications, Japan) 
pp. 253-256

Natural Scene Statistics of Color and Range

Che-Chun Su (The University of Texas at Austin, USA); Alan C Bovik (University of Texas at Austin, USA); Lawrence Cormack (The University of Texas at Austin, USA) pp. 257-260

\section{Biologically-Inspired Object Recognition System with Features From Complex} Wavelets

Tao Hong (Cambridge University, United Kingdom); Nick Geoffrey Kingsbury (University of Cambridge, United Kingdom); Michael D Furman (University of Cambridge, United Kingdom) pp. $261-264$

\section{Multiple Blind Re-Watermarking with Quantisation-Based Embedding} Jutta Hämmerle-Uhl (University of Salzburg, Austria); Christian Koidl (University of Salzburg, Austria); Andreas Uhl (Salzburg University, Austria) pp. 265-268

\section{Multi-resolution Level Set Image Segmentation Using Wavelet}

Fares Al-Qunaieer (University of Waterloo, Canada); Hamid R. Tizhoosh (University of Waterloo, Canada); Shahryar Rahnamayan (University of Ontario Institute of Technology (UOIT), Canada)

pp. 269-272

\section{Color Monogenic Wavelets for Image Analysis}

Raphael Soulard (University of Poitiers, France); Philippe Carré (University of Poitiers, France) pp. 273-276

An Effective Approach to Corner Point Detection Through Multiresolution Analysis Yang Bai (University of Tennessee Knoxville, USA) pp. $277-280$

Frequency Guided Bilateral Symmetry Gabor Wavelet Network Seungkyu Lee (Samsung Advanced Institute of Technology, Korea) pp. 281-284

\section{A Scale-Space Based Hierarchical Representation of Discrete Data} Moncef Hidane (Université de Caen Basse-Normandie \& ENSICAEN, CNRS, France); Olivier Lezoray (Université de Caen Basse-Normandie \& Greyc UMR CNRS 6072, France); Abderrahim Elmoataz (Université de Caen Basse-Normandi, France) pp. $285-288$

Putting Images on a Manifold for Atlas-based Image Segmentation Yihui Cao (Chinese Academy of Sciences, P.R. China); Xuelong Li (Xi'an Institute of Optics and Precision Mechanics, Chinese Academy of Sciences, P.R. China); Yuan Yuan (Chinese Academy of Sciences, P.R. China); Pingkun Yan (Chinese Academy of Sciences, P.R. China)

pp. 289-292 


\section{MA.PC: Image Coding (Poster)}

\section{Online Dictionaries for Image Prediction}

Mehmet Turkan (INRIA, France); Christine Guillemot (INRIA, France)

pp. 293-296

\section{Low-Complexity Image Coder/Decoder with an Approaching-Entropy Quad-Tree}

\section{Search Code for Embedded Computing Platforms}

Tao Ma (University of Nebraska-Lincoln, USA); Pradhumna L Shrestha (University of Nebraska-Lincoln, USA); Michael Hempel (University of Nebraska-Lincoln, USA); Dongming Peng (University Nebraska - Lincoln, USA); Hamid Sharif (University of Nebraska-Lincoln, USA)

pp. 297-300

\section{Preserving Sift Features in Jpeg-Encoded Images} Jianshu Chao (Technische Universität München, Germany); Eckehard Steinbach (Munich University of Technology, Germany) pp. 301-304

Very Low-Complexity Coding of Images Using Adaptive modulo-PCM Jose Prades-Nebot (Universitat Politècnica de València, Spain) pp. 305-308

Visually Lossless JPEG2000 At Fractional Resolutions Han Oh (University of Arizona, USA); Ali Bilgin (ECE Dept, The University of Arizona, USA); Michael W. Marcellin (ECE Dept, The University of Arizona, USA) pp. 309-312

JPEG XR Optimization with Graph-Based Soft Decision Quantization Yu Gao (Simon Fraser University, Canada); Duncan Chan (Simon Fraser University, Canada); Jie Liang (Simon Fraser University, Canada) pp. 313-316

Efficient Wavelet Packet Basis Selection in JPEG2000

Thomas Stütz (University of Salzburg, Austria); Andreas Uhl (Salzburg University, Austria)

pp. $317-320$

Decoder-Side Dimensionality Determination for Compressive-Projection Principal

Component Analysis of Hyperspectral Data

Wei Li (Mississippi State University, USA); James Fowler (Mississippi State University, USA) pp. 321-324

Improved Entropy Coding for Component-Based Image Coding Christian Feldmann (RWTH Aachen University, Germany); Johannes Ballé (RWTH Aachen University, Germany) pp. 325-328

3D-PMDC: A Parallelized Morphological Wavelet Codec for 3D Medical Datasets and Teleradiology Applications

Alberto Signoroni (University of Brescia, Italy); Mario Pezzoni (University of Brescia, Italy); Riccardo Leonardi (University of Brescia, Italy) pp. 329-332 
Probability Interval Partitioning Entropy Coding Using Systematic Variable-to-variable Length Codes

Heiner Kirchhoffer (Fraunhofer HHI, Germany); Detlev Marpe (Fraunhofer Institute for Telecommunications - Heinrich Hertz Institute, Germany); Christian Bartnik (Fraunhofer Heinrich-Hertz-Institute, Germany); Anastasia Henkel (Fraunhofer Heinrich-Hertz-Institute, Germany); Mischa Siekmann (Fraunhofer Heinrich Hertz Institute, Germany); Jan Stegemann (Fraunhofer Heinrich-Hertz-Institute, Germany); Heiko Schwarz (Fraunhofer HHI, Germany); Thomas Wiegand (HHI/FhG, Germany) pp. 333-336

An Adaptable Spatial-Temporal Error Concealment Method for Multiple Description Coding Based on Error Tracking

Meilin Yang (Purdue University, USA); Mary Comer (Purdue University, USA); Ed

Delp (Purdue University, USA)

pp. 337-340

\title{
Coupled Distributed Arithmetic Coding
}

Xi Chen (University of New South Wales, Australia); David Taubman (University of New South Wales, Australia)

pp. 341-344

\section{MA.PD: Hardware and GPU Issues in Video / High Dynamic Range Imaging and Shape Estimation (Poster)}

\author{
Curve-Based and Image-based JND Contrast Analysis for Inverse Tone Mapping \\ Operators \\ Ching-Te Chiu (National Tsing Hua University, Taiwan) \\ pp. $345-348$
}

Hybrid Light Coding for Fast and High-accuracy Shape Acquisition

Lulu He (Northwestern University, USA); Sen Wang (Eastman Kodak Company, USA); Paul J Kane (Eastman Kodak Company, USA); Thrasyvoulos N. Pappas (Northwestern University, USA)

pp. 349-352

Motion Artifact-free HDR Imaging Under Dynamic Environments

Sungchan Park (Samsung Electronics, Samsung Advanced Institute of Technology, Korea); Hyun-Hwa Oh (Samsung Electronics, Samsung Advanced Institute of Technology, Korea); Jae-Hyun Kwon (Samsung Advanced Institute of Technology, Korea); Wonhee Choe (Samsung Electronics, Samsung Advanced Institute of Technology, Korea)

pp. $353-356$

Geometrical Transformation-Based Ghost Artifacts Removing for High Dynamic Range Image Jaehyun Im (Chung-Ang University, Korea); Sangsik Jang (Chung-Ang University, Korea); Seungwon Lee (Chung-Ang University, Korea); Joonki Paik (Chung-Ang University, Korea)

pp. 357-360 
Fully Automated Exposure Fusion Algorithm for Mobile Platforms

Tomislav Kartalov (Faculty of Electrical Engeneering and Information Technologies Skopje, Macedonia); Zoran Ivanovski (Ss. Cyril and Methodius University, Macedonia); Ljupcho Panovski (Faculty of Electrical Engeneering and Information Technologies - Skopje, Macedonia)

pp. 361-364

Fast Movement Detection for High Dynamic Range Imaging

Zhengguo Li (Institute for Inforcomm Research, Singapore); Zijian Zhu (Institute for Infocomm Research, Singapore); Susanto Rahardja (Institute for Infocomm Research, Singapore)

pp. $365-368$

One-Round Renormalization Based 2-bin/Cycle H.264/AVC CABAC Encoder Zhenyu Liu (Tsinghua University, P.R. China); Dongsheng Wang (Tsinghua University, P.R. China)

pp. 369-372

An Efficient VLSI Architecture for $4 \times 4$ Intra Prediction in the High Efficiency Video Coding (HEVC) Standard

Fu Li (Xidian University, P.R. China); Guangming Shi (Xidian University, P.R. China); Feng Wu (Microsoft Research Asia, P.R. China) pp. 373-376

Ultra High Definition Video Decoding with Motion JPEG XR Using the GPU Bart Pieters (Ghent University - IBBT - Multimedia Lab, Belgium); Jan De Cock (Ghent University - IBBT, Belgium); Charles Hollemeersch (Ghent University - IBBT, Belgium); Jeroen Wielandt (Ghent University - IBBT, Belgium); Peter Lambert (Ghent University - IBBT, Belgium); Rik Van de Walle (Ghent University - IBBT, Belgium) pp. $377-380$

H.264/AVC UHD Decoder Implementation on Multi-Cluster Platform Using Hybrid Parallelization Method

Sangjo Lee (Samsung, Korea)

pp. 381-384

A Novel Energy Reduction Technique for H.264 Intra Mode Decision

Yusuf Adibelli (Sabancı University, Turkey); Mustafa Parlak (Sabancı University, Turkey); Ilker Hamzaoglu (Sabanci University, Turkey)

pp. 385-388

\section{Fast FPGA-Based Architecture for Pedestrian Detection Based on Covariance} Matrices

Samuele Martelli (University of Verona, Italy); Diego Tosato (University of Verona, Italy); Marco Cristani (University of Verona \& Istituto Italiano di Tecnologia, Italy); Vittorio Murino (University of Verona, Italy) pp. 389-392

A High-Throughput Parallel Hardware Architecture for H.264/AVC CAVLC Encoding Muhammad Shafique (Karlsruhe Institute of Technology (KIT), Germany); Adnan Tüfek (Karlsruhe Institute of Technology, Germany); Jörg Henkel (Universität karlsruhe (TH), Germany)

pp. 393-396 
ReVC: Computationally Reliable Video Coding on Unreliable Hardware Platforms: A Case Study on Error-Tolerant H.264/AVC CAVLC Entropy Coding

Semeen Rehman (Karlsruhe Institute of Technology, Germany); Muhammad Shafique (Karlsruhe Institute of Technology (KIT), Germany); Florian Kriebel (Karlsruhe Institute of Technology, Germany); Jörg Henkel (Universität karlsruhe (TH), Germany) pp. $397-400$

\section{MA.PE: CT Image Processing Methods (Poster)}

\section{Synthetic OCT Data for Image Processing Performance Testing}

Pedro Serranho (University of Coimbra, Portugal); Cristina Maduro (AIBILI \& CNTM, Portugal); Torcato Santos (AIBILI, Portugal); José Cunha-Vaz (IBILI, Fac. Medicine, University Coimbra \& AIBILI, Portugal); Rui Bernardes (Inst.

Biophysics\&Biomathematics, IBILI, Fac. Medicine, University Coimbra \& Center New Technologies for Medicine, AIBILI, Coimbra, Portugal, Portugal)

pp. 401-404

\section{A Comparative Evaluation of Ring Artifacts Reduction Filters for X-ray Computed} Microtomography Images

Francesco Brun (University of Trieste \& Sincrotrone Trieste S.C.p.A., Italy); Georgios Kourousias (Sincrotrone Trieste S.C.p.A, Italy); Diego Dreossi (Sincrotrone Trieste S. C. p. A., Italy); Lucia Mancini (Sincrotrone Trieste S.C.p.A., Italy); Giuliana Tromba (Sincrotrone Trieste S. C. p. A., Italy) pp. 405-408

\section{A Novel Alternative Algorithm for Limited Angle Tomography}

Xiaoqiang Lu (Chinese Academy of Sciences, P.R. China); Yuan Yuan (Chinese Academy of Sciences, P.R. China); Pingkun Yan (Chinese Academy of Sciences, P.R. China); Xuelong Li (Xi'an Institute of Optics and Precision Mechanics, Chinese Academy of Sciences, P.R. China) pp. 409-412

\section{A Novel Coupled Transmission-Reflection Tomography and the V-line Radon} Transform

Rémi Régnier (ETIS/ENSEA/CNRS/University of Cergy-Pontoise, France); Maï Nguyen-Verger (University of Cergy-Pontoise, France) pp. 413-416

PET Image Reconstruction: GPU-Accelerated Particle Filter Framework Fengchao Yu (Zhejiang University, P.R. China); Huafeng Liu (Zhejiang University, P.R. China); Pengcheng Shi (, USA) pp. $417-420$

\section{Sensitivity to Error of the Truncated Hilbert Transform Technique for Interior}

\section{Reconstruction}

Alex Opie (University of Canterbury, New Zealand); Phil Bones (University of Canterbury, New Zealand)

pp. 421-424 
Interactive CT Image Segmentation with Online Discriminative Learning

Wei Yang (Sun Yat-Sen University, P.R. China); Xiaolong Wang (Sun Yat-Sen

University, P.R. China); Liang Lin (Sun Yat-Sen University, P.R. China); Chengying

Gao (Sun Yat-Sen University, P.R. China)

pp. $425-428$

An Algorithm for Simultaneous Image Segmentation and Nonrigid Registration, with

Clinical Application in Image Guided Radiotherapy

Chao Lu (Yale University, USA); Jingjing Zhu (Yale University, USA); James Duncan

(Yale University, USA)

pp. 429-432

Integrated Framework for Simultaneous Segmentation and Registration of Carpal

Bones

Xin Chen (The University of Manchester, United Kingdom); Jim Graham (The

University of Manchester, United Kingdom); Charles Hutchinson (University of

Warwick, United Kingdom)

pp. 433-436

Automated Delineation of Tree-Rings in X-ray Computed Tomography Images of Wood Philippe Borianne (Umr Amap \& CIRAD, France); Gerard Subsol (Lirmm, France) pp. 437-440

Multiscale Sparse Representation of High-Resolution Computed Tomography (HRCT) Lung Images for Diffuse Lung Disease Classification

Kiet T. Vo (University of New South Wales, Australia)

pp. 441-444

Modeling a Parallelism Constraint in Active Contours. Application to the

Segmentation of Eye Vessels and Retinal Layers

Itebeddine Ghorbel (Télécom ParisTech, France); Florence Rossant (ISEP, France);

Isabelle Bloch (Télécom ParisTech, France); Michel Paques (Clinical Investigation

Center 503, France)

pp. 445-448

Sparsity-based Retinal Layer Segmentation of Optical Coherence Tomography Images Jason Tokayer (University of Southern California \& Center for Ophthalmic Optics and Lasers, USA); Antonio Ortega (USC, USA); David Huang (Oregon Health and Sciences University, USA)

pp. $449-452$

Direct Reconstruction of Parametric Images From Cardiac Gated Dynamic SPECT Data

Xiaofeng Niu (Illinois Institute of Technology, USA); Yongyi Yang (Illinois Institute of Technology, USA); Miles Wernick (IIT, USA)

pp. 453-456

Effects of Piecewise Smoothing on Cardiac SPECT Reconstruction

Wenyuan Qi (Illinoise Institute of Technology, USA); Xiaofeng Niu (Illinois Institute of Technology, USA); Yongyi Yang (Illinois Institute of Technology, USA) pp. $457-460$

A New 3D Paradigm for Metal Artifact Reduction in Dental CT

Valery Naranjo (Universidad Politecnica de Valencia, Spain); Roberto Lloréns

(Universidad Politécnica de Valencia, Spain); Mariano Alcañiz (Universidad 
Politécnica de Valencia, Spain); Rafael Verdú-Monedero (Universidad Politécnica de Cartagena, Spain); Jorge Larrey-Ruiz (Universidad Politécnica de Cartagena, Spain); Juan Morales-Sánchez (Universidad Politécnica de Cartagena, Spain) pp. 461-464

\section{MA.PF: Tracking (Poster)}

\section{An Improved Occlusion Handling for Appearance-Based Tracking}

Gwo-Cheng Chao (National Taiwan University, Taiwan); Shyh-Kang Jeng (National Taiwan University, Taiwan); Shung-Shing Lee (Ching Yun University, Taiwan) pp. 465-468

\section{A Snake Algorithm for Automatically Tracking Multiple Objects}

Hua Fang (PAICHAI University, Korea); Shin-Hyoung Kim (AR VISION Inc., Korea); Jong Whan Jang (PAICHAI University, Korea)

pp. 469-472

\section{Mean-shift Tracking Algorithm with Weight Fusion Strategy}

Lingfeng Wang (National Laboratory of Pattern Recognition, P.R. China); Chunhong Pan (Institute of Automation, Chinese Academy of Sciences, P.R. China); Shiming Xiang (Institute of Automation, Chinese Academy of Sciences, P.R. China) pp. 473-476

\section{Complementary Visual Tracking}

Shu Wang (Dalian University of Technology, P.R. China); Huchuan Lu (Dalian University of Technology, P.R. China); Guang Yang (Dong Cai Science and Technology, P.R. China) pp. 477-480

\section{A Cost Function Approach for Multi-Human Tracking} Yuan Shen (Beijing Jiaotong University, P.R. China); Zhenjiang Miao (Institute of Information Science, Beijing Jiaotong University, P.R. China); Zhifei Wang (Institute of Information Science, Beijing Jiaotong University, P.R. China) pp. $481-484$

\section{Robust Visual Tracking Via Transfer Learning}

Wenhan Luo (National Lab of Pattern Recognition, Institute of Automation, CAS, P.R. China); Xi Li (University of Adelaide, Australia); Wei Li (National Lab of Pattern Recognition, Institute of Automation, CAS, P.R. China); Weiming Hu (CAS, P.R. China) pp. $485-488$

\section{Visual Tracking Using Compensated Motion Model for Mobile Cameras} Jian-Yi Lu (Evest Corp., Taiwan); Yi-Chun Wei (National Central University, Taiwan); Chih-Wei Tang (National Central University, Taiwan) pp. 489-492

\section{Region Tracking with Narrow Perception of Background}

Julien Mille (Université de Lyon, LIRIS \& Université Lyon 1, France); Jean-Loïc Rose (Université de LYON, CNRS \& LIRIS, France)

pp. 493-496 
Adaptive Multi-Resolution CRF-based Contour Tracking

Fatemeh Moayedi (University of Shiraz, Iran); Zohreh Azimifar (Shiraz University, Iran); Paul Fieguth (University of Waterloo, Canada); Alireza Kazemi (University of Shiraz, Iran)

pp. 497-500

Monte Carlo Sampling for Visual Pose Tracking

Jehoon Lee (Georgia Institute of Technology, USA); Romeil Sandhu (Georgia Institute of Technology, USA); Allen Tannenbaum (Georgia Institute of Technology, USA) pp. 501-504

SnooperTrack: Text Detection and Tracking for Outdoor Videos

Rodrigo Minetto (University of Campinas \& University Pierre and Marie Curie, Brazil); Nicolas Thome (University Pierre et Marie Curie, France); Matthieu Cord (UPMC Paris 6, France); Neucimar Leite (State University of Campinas, Brazil); Jorge Stolfi (University of Campinas, Brazil)

pp. 505-508

\title{
Robust Visual Tracking Via Context Objects Computing
} Zhongqian Sun (Harbin Institute of Technology, P.R. China); Hongxun Yao (Harbin Institute of Technology, P.R. China); Shengping Zhang (Harbin Institute of Technology, P.R. China); Xin Sun (Harbin Institute of Technology, P.R. China) pp. 509-512

\section{Particle-Based Tracking Model for Automatic Anomaly Detection} Erwan Jouneau (Multitel, Belgium); Cyril Carincotte (Multitel, Belgium) pp. 513-516

\section{Robust Visual Tracking Via Ranking SVM}

Yancheng Bai (National Lab of Pattern Recognition, Institute of Automation, Chinese Academy of Sciences, P.R. China); Ming Tang (Chinese Academy of Sciences, P.R. China)

pp. $517-520$

Vanishing Point-Based Line Sampling for Efficient Axis-Based People Localization Kuo-Hua Lo (National Chiao Tung University, Taiwan); Jen-Hui Chuang (National Chiao Tung University, Taiwan)

pp. 521-524

\section{MA.PG: Preprocessing and Features for Biometrics (Poster)}

\author{
A Markerless Motion Capture System with Automatic Subject-Specific Body Model \\ Acquisition and Robust Pose Tracking From 3D Data \\ Zheng Zhang (Nanyang Technological University, Singapore); Seah Hock Soon \\ (Nanyang Technological University, Singapore); Chee Kwang Quah (Nanyang \\ Technological University, Singapore); Jixiang Sun (National University of Defense \\ Technology, Singapore) \\ pp. $525-528$
}

Robust Body Parts Tracking Using Particle Filter and Dynamic Template Matilde Gonzalez (Université Paul Sabatier, France); Christophe Collet (Université Paul Sabatier, France) 
pp. $529-532$

Action Recognition Using Partial Least Squares and Support Vector Machines Samah Ramadan (University Of Maryland, USA); Larry S Davis (University of Maryland, USA)

pp. 533-536

\section{Augmented Fingerprint Minutiae Vicinity}

Bian Yang (Gjøvik University College, Norway); Christoph Busch (Gjøvik University College, Norway)

pp. 537-540

\section{Multi-view Multi-stance Gait Identification}

Maodi Hu (Beihang University, P.R. China); Yunhong Wang (Beihang University, P.R. China); Zhaoxiang Zhang (Beihang University, P.R. China); De Zhang (Beihang University, P.R. China)

pp. 541-544

\section{Speed-Invariant Gait Recognition Based on Procrustes Shape Analysis Using Higher-}

Order Shape Configuration

Worapan Kusakunniran (University of New South Wales \& National ICT Australia, Australia); Qiang Wu (University of Technology, Sydney, Australia); Jian Zhang (The University of New South Wales \& NICTA, Australia); Hongdong Li (Australian National University, Australia)

pp. 545-548

\section{Hybrid Hand Tracking System}

Jing-Ming Guo (National Taiwan University of Science and Technology, Taiwan); Hoang-Son Nguyen (National Taiwan University of Science and Technology, Taiwan) pp. 549-552

\section{Exploiting Color SIFT Features for 2D Ear Recognition}

Jindan Zhou (University of Miami, USA); Steven Cadavid (University of Miami, USA); Mohamed Abdel-Mottaleb (University of Miami, USA)

pp. 553-556

\section{GPU-based Face Tracking At 500 Fps}

Idaku Ishii (Hiroshima University, Japan); HIroki Ichida (Hiroshima University, Japan); Takeshi Takaki (Hiroshima University, Japan) pp. 557-560

\section{A Comparison of Techniques for Robust Gender Recognition}

Richard Rojas-Bello (Universidad Autónoma de Madrid, Spain); Luis Lago-Fernández (Universidad Autónoma de Madrid, Spain); Gonzalo Martínez-Muñoz (Universidad Autónoma de Madrid, Spain); Manuel Sánchez-Montañés (Universidad Autónoma de Madrid, Spain)

pp. 561-564

\section{Multi-task GLOH Feature Selection for Human Age Estimation}

Yixiong Liang (Central South University, P.R. China); LIngbo Liu (Central South University, P.R. China); Ying Xu (Central South University, P.R. China); Yao Xiang (Central South University, P.R. China); Beiji Zou (Central South University, P.R. China) pp. 565-568 


\section{Pose Invariant Facial Component-Landmark Detection}

Boris Efraty (University of Houston, USA); Manos Papadakis (TLC2, USA); Adam

Profitt (TLC2, USA); Shishir Shah (University of Houston, USA); Ioannis Kakadiaris (University of Houston, USA)

pp. 569-572

\section{Spatial and Probabilistic Codebook Template Based Head Pose Estimation From} Unconstrained Environments

Meltem Demirkus (Mcgill University, Canada); Boris N Oreshkin (Mcgill University, Canada); James Clark (McGill University, Canada); Tal Arbel (McGill, Canada) pp. 573-576

Newton Optimization Based Congealing for Facial Image Alignment Weiyuan Ni (Grenoble University, France); Alice Caplier (Institut National Polytechnique de Grenoble, France) pp. $577-580$

\section{MP.L1: Compression of High-Dimensional Media Data for Interactive Navigation (Special Session)}

\section{Efficient Communication of Video Using Metadata}

Aous T. Naman (University of New South Wales, Australia); Duncan Edwards (University of New South Wales, Australia); David Taubman (University of New South Wales, Australia) pp. 581-584

Mapping Data on a Rotated Grid in High-dimensions for Lossless Compression Zihong Fan (University of Southern California, USA); Antonio Ortega (USC, USA) pp. 585-588

Interactive Multiview Video System with Low Decoding Complexity Thomas Maugey (Ecole Polytechnique Fédérale de Lausanne, Switzerland); Pascal Frossard (Swiss Federal Institute of Technology - EPFL, Switzerland) pp. 589-592

Frame Structure Optimization for Interactive Multiview Video Streaming with Bounded Network Delay

Xiaoyu Xiu (Simon Fraser University, Canada); Gene Cheung (National Institute of Informatics, Japan); Jie Liang (Simon Fraser University, Canada) pp. 593-596

break

Using Distributed Source Coding and Depth Image Based Rendering to Improve Interactive Multiview Video Access

Giovanni Petrazzuoli (Télécom ParisTech, France); Marco Cagnazzo (TELECOM

ParisTech, France); Frederic Dufaux (Telecom Paristech \& CNRS, France); Beatrice Pesquet-Popescu (Télécom ParisTech, France)

pp. 597-600 


\section{Interactive Multiview Image Coding}

Andriy Gelman (Imperial College London, United Kingdom); Pier Luigi Dragotti (Imperial College London, United Kingdom); Vladan Velisavljević (University of Bedfordshire, United Kingdom) pp. 601-604

\section{A Trellis-based Approach for Robust View Synthesis}

Dong Tian (Mitsubishi Electric Research Labs, USA); Anthony Vetro (Mitsubishi Electic Research Laboratories, USA); Matthew Brand (MERL, USA) pp. 605-608

Adaptive pixel/patch-based Synthesis for Texture Compression Fabien Racape (Technicolor Research \& Innovation \& Institut Electronique Telecommunications de Rennes, France); Simon Lefort (Technicolor Research \& Innovation, France); Edouard Francois (Technicolor, France); Marie Babel (IETR / INSA Rennes, France); Olivier Deforges (IETR / INSA Rennes, France) pp. 609-612

\section{MP.L2: Image \& Video Sensing (Lecture)}

Single Image Local Blur Identification

Pauline Trouvé (Onera \& DGA, France); Frédéric Champagnat (ONERA, France);

Guy Le Besnerais (ONERA, France); Jérôme Idier (IRCCyN, France)

pp. 613-616

\section{Lens Distortion Correction with a Calibration Harp}

Rafael Grompone von Gioi (CMLA, ENS-Cachan, France); Pascal Monasse

(IMAGINE, LIGM-Universite Paris Est, France); Jean-Michel Morel (CMLA, ENS-

Cachan, France); Zhongwei Tang (CMLA, ENS-Cachan, France)

pp. 617-620

\section{Effective Autofocus Decision Using Reciprocal Focus Profile}

Dong-Chen Tsai (National Taiwan University, Taiwan); Homer Chen (National Taiwan University, Taiwan)

pp. 621-624

\section{Automatic Video Deshearing for Skew Sequences Captured by Rolling Shutter Cameras}

Dũng Trung Võ (Samsung Information Systems America ( Samsung Electronics US R\&D Center ), USA); Surapong Lertrattanapanich (Digital Media Solutions Lab, Samsung Electronics US R\&D Center, USA); Yeong-Taeg Kim (Samsung SISA, USA) pp. $625-628$

break

\section{Robust Video Stabilization Approach Based on a Voting Strategy}

Giovanni Puglisi (University of Catania, Italy); Sebastiano Battiato (University of Catania, Italy)

pp. 629-632 
An Auto-Focus Sharpness Function for Stereo Image Pairs

Mohammad Rahman (University of Texas at Dallas, USA); Nasser Kehtarnavaz (University of Texas at Dallas, USA); Siamak Yousefi (University of Texas at Dallas, USA)

pp. 633-636

Calibration of Central Catadioptric Camera with One-Dimensional Object Undertaking General Motions

Xiaoming Deng (Institute of Software, Chinese Academy of Sciences, P.R. China); Fuchao Wu (NLPR, P.R. China); Yihong Wu (Chinese Academy of Sciences, P.R. China); Liang Chang (Beijing Normal University, P.R. China); Wei Liu (ISCAS, P.R. China); Hongan Wang (Chinese Academy of Sciences, P.R. China) pp. 637-640

Paracatadioptric Camera Calibration Using Sphere Images Huixian Duan (Chinese Academy of Sciences, P.R. China); Yihong Wu (Chinese Academy of Sciences, P.R. China) pp. 641-644

\section{MP.L3: Saliency and Object Recognition (Lecture)}

\section{Visual Saliency Detection Based on Bayesian Model}

Yulin Xie (Dalian University of Technology, P.R. China); Huchuan Lu (Dalian University of Technology, P.R. China)

pp. 645-648

High Resolution Biologically Inspired Salient Region Detection

Yusuf Saber (Ryerson University, Canada); Matthew J Kyan (Ryerson University, Canada)

pp. 649-652

\section{Simultaneous Detection and Segmentation for Generic Objects}

Albert Torrent (University of Girona, Spain); Xavier Lladó (University of Girona, Spain); Jordi Freixenet (University of Girona, USA); Antonio Torralba (Massachusetts Institute of Technology, USA)

pp. $653-656$

\section{Sparse Representation Based Visual Element Analysis}

Xue Li (Harbin Institute of Technology, P.R. China); Hongxun Yao (Harbin Institute of Technology, P.R. China); Xiaoshuai Sun (Harbin Institute of Technology, P.R. China); Rongrong Ji (Columbia University, P.R. China); Xianming Liu (Harbin Institute of Technology, P.R. China); Pengfei Xu (Harbin Institute of Technology, P.R. China) pp. 657-660

break

\section{Spatial Coordinate Coding to Reduce Histogram Representations, Dominant Angle} and Colour Pyramid Match

Piotr Koniusz (University of Surrey, United Kingdom); Krystian Mikolajczyk (University of Surrey, United Kingdom)

pp. 661-664 
Multivariate log-Gaussian Cox Models of Elementary Shapes for Recognizing Natural Scene Categories

Huu-Giao Nguyen (Institut Telecom / Telecom Bretagne / Labsticc, France)

pp. 665-668

Improving Image Similarity with Vectors of Locally Aggregated Tensors

David Picard (ETIS - ENSEA, France); Philippe H Gosselin (CNRS, ENSEA, UniversityCergy-Pontoise, France)

pp. 669-672

Recognizing 3D Objects in Cluttered Scenes Using Projection Images

Dimitrios Zarpalas (Informatics and Telematics Institute, Greece); Georgios Kordelas (Informatics and Telematics Institute, Greece); Petros Daras (Informatics \& Telematics Institute, Greece)

pp. 673-676

\section{MP.L4: Image Restoration (Lecture)}

Single Image Spatially Variant Out-Of-Focus Blur Removal

Stanley Chan (University of California, San Diego, USA); Truong Nguyen (University of California in San Diego, USA)

pp. 677-680

Patch-based Image Deconvolution Via Joint Modeling of Sparse Priors Chao Jia (The University of Texas at Austin, USA); Brian L Evans (The University of Texas at Austin, USA)

pp. 681-684

Variational Image Restoration Based on Poisson Singular Integral and Curvelet-Type Decomposition Space Regularization

Lili Huang (Nanjing University of Science and Technology, P.R. China); Liang Xiao (Nanjing University of Science and Technology, P.R. China); Zhihui Wei (Nanjing University of Science and Technology, P.R. China); Zhengrong Zhang (Nanjing University of Science and Technology, P.R. China) pp. $685-688$

Two Constrained Formulations for Deblurring Poisson Noisy Images Mikael Carlavan (INRIA, France); Laure Blanc-Féraud (CNRS, France) pp. 689-692

break

A Convex Minimization Model in Image Restoration Via One-Dimensional Sobolev Norm Profiles

Yunho Kim (University of California Irvine, USA); John Garnett (University of California Los Angeles, USA); Luminita Vese (University of California, Los Angeles, USA)

pp. $693-696$ 
Adaptive Regularization for Multiple Image Restoration Using an Extended Total Variations Approach

Matthew A. Kitchener (University of Wollongong, Australia); Abdesselam Bouzerdoum (University of Wollongong, Australia); Son Lam Phung (University of Wollongong, Australia) pp. $697-700$

\section{A New Image Deblurring Algorithm with Less Ringing Artifacts Via Error Variance} Estimation and Soft Decision

Ruiqin Xiong (Peking University, P.R. China)

pp. 701-704

\section{A Second-order Extension of TV Regularization for Image Deblurring}

Zafer Dogan (Ecole Polytechnique Federale de Lausanne, Switzerland); Stamatios Lefkimmiatis (Ecole Polytechnique Federale de Lausanne, Switzerland); Aurélien Bourquard (Ecole Polytechnique Federale de Lausanne, Switzerland); Michael Unser (EPFL, Switzerland)

pp. 705-708

\section{MP.L5: Best Student Paper Award Session (Lecture)}

\section{MP.L6: Biomedical Image Shape Segmentation (Lecture)}

\section{Learning-Based Non-Rigid Image Registration Using Prior Joint Intensity \\ Distributions with Graph-Cuts}

Ronald W. K. So (Hong Kong University of Science and Technology, Hong Kong);

Albert C. S. Chung (The Hong Kong University of Science and Technology, Hong Kong)

pp. $709-712$

\section{A Probabilistic Framework for Automatic Prostate Segmentation with a Statistical Model of Shape and Appearance}

Soumya Ghose (Université de Bourgogne \& Universitat de Girona, France); Arnau Oliver (University of Girona, Spain); Robert Marti (University of Girona, Spain); Xavier Lladó (University of Girona, Spain); Jordi Freixenet (University of Girona, USA); Joan Vilanova (Clinica Girona, Spain); Fabrice Meriaudeau (University of Bourgogne, France) pp. $713-716$

\section{A New Shape Based Segmentation Framework Using Statistical and Variational Methods}

Melih Aslan (CVIP Lab, University of Louisville, USA); Hossam Abdelmunim (Faculty of Engineering, Ain Shams University, ? ); Aly Farag (University of Louisville, USA); Ben Arnold (Image Analysis, Inc., USA); Eslam Mostafa (University of Louisville, USA); Ping Xiang (Image Analysis, Inc., USA) pp. $717-720$ 
Segmenting Human Knee Cartilage Automatically From Multi-contrast MR Images Using Support Vector Machines and Discriminative Random Fields

Kunlei Zhang (Nanyang Technological University, Singapore); Jun Deng (Nanyang Technological University, Singapore); Lu Wenmiao (Nanyang Technological

University, Singapore)

pp. 721-724

break

A Fuzzy Framework with Prior Information Unifying Registration, Segmentation, and Bias Field Correction of Brain MRI

Moumen El-Melegy (Assiut University, Egypt); Aly Farag (University of Louisville, USA)

pp. $725-728$

Elastic Shape Registration Using an Incremental Free Form Deformation Approach with the ICP Algorithm

Hossam Abdelmunim (Faculty of Engineering, Ain Shams University, ? ); Aly Farag (University of Louisville, USA)

pp. $729-732$

\section{MP.L7: Reduced-Complexity Video Coding (Lecture)}

HEVC ALF Decode Complexity Analysis and Reduction

Madhukar Budagavi (Texas Instruments, USA); Vivienne Sze (Texas Instruments, USA); Minhua Zhou (Texas Instruments, USA)

pp. $733-736$

Low Complexity Deblocking Filter Perceptual Optimization for the HEVC Codec Matteo Naccari (British Broadcasting Corporation - Research and Development, United Kingdom); Catarina Brites (IST - IT, Portugal); Joao Ascenso (ISEL \& IT, Portugal); Fernando Pereira (IST-IT, Portugal) pp. $737-740$

Complexity-Aware Adaptive Spatial Pre-Processing for Roi Scalable Video Coding with Dynamic Transition Region

Dan Grois (Ben-Gurion University of the Negev, Israel); Ofer Hadar (Ben-Gurion University of the Negev, Israel)

pp. 741-744

Video Compression Complexity Reduction with Adaptive Down-Sampling

Diogo Garcia (Universidade de Brasília, Brazil); Tiago da Fonseca (Universidade de Brasília, Brazil); Ricardo L de Queiroz (University of Brasil, Brazil) pp. 745-748

break

A Multi-Level Dynamic Complexity Reduction Scheme for Multiview Video Coding Bruno Zatt (Federal University of Rio Grande do Sul, Brazil); Muhammad Shafique (Karlsruhe Institute of Technology (KIT), Germany); Sergio Bampi (Federal University of Rio Grande do Sul \& Microelectronics Group at UFRGS, Brazil); Jörg Henkel (Universität karlsruhe (TH), Germany) 
pp. $749-752$

Reduced-complexity Entropy Coding of Transform Coefficient Levels Using Truncated Golomb-Rice Codes in Video Compression

Tung Nguyen (Fraunhofer HHI, Germany); Detlev Marpe (Fraunhofer Institute for Telecommunications - Heinrich Hertz Institute, Germany); Heiko Schwarz (Fraunhofer $\mathrm{HHI}$, Germany); Thomas Wiegand (Fraunhofer Institute for Telecommunications Heinrich-Hertz-Institute, Germany) pp. 753-756

Frame Buffer Compression for Low-power Video Coding Zhan Ma (Samsung Telecommunications America, USA); Andrew Segall (Sharp Labs, USA) pp. $757-760$

\section{MP.L8: Face Recognition (Lecture)}

Locality-constrained Group Sparse Representation for Robust Face Recognition Yu-Wei Chao (Academia Sinica, Taiwan); Yi-Ren Yeh (Research Center for Information Technology Innovation, Academia Sinica, Taipei, Taiwan); Yu-Wen Chen (National Taiwan University, Taiwan); Yuh-Jye Lee (National Taiwan University of Science and Technology, Taiwan); Yu-Chiang Frank Wang (Academia Sinica, Taiwan) pp. 761-764

Face Recognition Using Multi-Scale Local Phase Quantisation and Linear Regression Classifier

Muhammad Atif Tahir (University of Surrey \& University of Northumbria, United Kingdom); C. H. Chan (University of Surrey, United Kingdom); Josef Kittler (University of Surrey, United Kingdom); Ahmed Bouridane (Northumbria University, United Kingdom)

pp. $765-768$

MAP-MRF Based Lip Segmentation Without True Segment Number Yiu-ming Cheung (Hong Kong Baptist University, Hong Kong); Meng Li (Hong Kong Baptist University, Hong Kong) pp. 769-772

3D Facial Expression Recognition Using Zernike Moments on Depth Images Nicholas Vretos (Aristotle University of Thessaloniki, Greece); Nikos Nikolaidis (Aristotle University of Thessaloniki \& Informatics and Telematics Institute, CERTH, Greece); Ioannis Pitas (Aristotle University of Thessaloniki, Greece) pp. $773-776$

break

Illumination Robust Dictionary-based Face Recognition

Vishal Patel (University of Maryland, USA); Tao Wu (University of Maryland, USA); Soma Biswas (University of Notre Dame, USA); P. Jonathon Phillips (NIST, USA); Rama Chellappa (University of Maryland, USA) pp. $777-780$ 
Face Tracking in Low Resolution Videos Under Illumination Variations Wilman W.W. Zou (Hong Kong Baptist University, Hong Kong); Rama Chellappa (University of Maryland, USA); Pong C Yuen (Hong Kong Baptist University, Hong Kong) pp. $781-784$

\title{
Color HOG-EBGM for Face Recognition
}

David Monzo (Universidad Politecnica de Valencia, Spain); Alberto Albiol (Universidad Politecnica de Valencia, Spain); Antonio Albiol (Universidad Politecnica Valencia, Spain); Jose M. Mossi (Polytechnic University of Valencia, Spain) pp. $785-788$

Feature Selection Via Simultaneous Sparse Approximation for Person Specific Face Verification

Yixiong Liang (Central South University, P.R. China); Lei Wang (Central South University, P.R. China); Shenghui Liao (Central South University, P.R. China); Beiji

Zou (Central South University, P.R. China)

pp. 789-792

\section{MP.PA: Geometry Texture and Object-based Representation (Poster)}

\author{
Tensor-Directed Simulation of Strokes for Image Stylization with Hatching and \\ Contours \\ David Tschumperlé (Laboratoire GREYC (CNRS UMR 6072) \& Equipe Image, \\ France) \\ pp. 793-796
}

Pattern Recognition by Affine Legendre Moment Invariants

Hui Zhang (University of Windsor, Canada); Jonathan Wu (University of Windsor, Canada)

pp. $797-800$

\section{Spatiogram Features to Characterize Pearls in Paintings}

Ljiljana Platiša (Ghent University, Belgium); Bruno Cornelis (Vrije Universiteit Brussel, Belgium); Tijana Ružic (Ghent University, Belgium); Aleksandra Pižurica (Ghent University, Belgium); Ann Dooms (Vrije Universiteit Brussel, Belgium); Maximiliaan Martens (Ghent University, Belgium); Marc De Mey (The Flemish Academic Centre for Science and the Arts, Belgium); Ingrid Daubechies (Princeton University, USA) pp. 801-804

MPL-Boosted Integrable Features Pool for Pedestrian Detection Junqiang Wang (Beijing University of Posts and Telecommunications, P.R. China); Huadong Ma (Beijing University of Posts and Telecommunications, P.R. China) pp. $805-808$

\section{A Combined Texture-Shape Descriptor for Enhanced 3D Feature Matching}

Federico Tombari (University of Bologna, Italy); Samuele Salti (University of Bologna, Italy); Luigi Di Stefano (Universita' di Bologna, Italy) pp. 809-812 
Moving Object Selection Based on an Active Curve Approach

Marwen Nouri (University of Paris Descartes \& Alcatel-Lucent Bell Labs France, France); Emmanuel Marilly (Alcatel Lucent Bell Labs France, France); Nicole Vincent (Université Paris 5, France)

pp. 813-816

Image Analysis Using Separable Two-Dimensional Discrete Orthogonal Moments Hongqing Zhu (East China University of Science and Technolog, P.R. China) pp. $817-820$

Ensemble of Furthest Subspace Pairs for Enhanced Image Set Matching Mehrtash T. Harandi (NICTA \& University of Queensland, Australia); Conrad Sanderson (NICTA, Australia); Abbas Bigdeli (National ICT Australia, Australia); Brian C Lovell (NICTA, Australia) pp. 821-824

From Universal Bag-of-Words to Adaptive Bag-of-Phrases for Mobile Scene Recognition

Tao Chen (Nanyang Technological University, Singapore); Kim Hui Yap (Nanyang Technological University, Singapore); Lap-Pui Chau (Nanyang Technological University, Singapore) pp. 825-828

Generic Polar Harmonic Transforms for Invariant Image Description Thai V. Hoang (LORIA, Université Nancy 2, France); Salvatore Tabbone (University Nancy 2, France) pp. 829-832

\section{Combining Sorted Random Features for Texture Classification}

Li Liu (National University of Defense Technology, P.R. China); Paul Fieguth (University of Waterloo, Canada); Gangyao Kuang (National University of Defense Technology, P.R. China)

pp. 833-836

LSP: Local Similarity Pattern, a New Approach for Rotation Invariant Noisy Texture Analysis Hamid Reza Pourreza (Ferdowsi University of Mashad, Iran); Mina Masoudifar (Sabzevar Tarbiat Moallem University, Iran); MohammadMahdi ManafZade (Ferdowsi University, Iran) pp. 837-840

Local Binary Pattern Histogram Based Texton Learning for Texture Classification Yonggang He (Huazhong University of Science and Technology, P.R. China); Nong Sang (Huazhong University of Science and Technology, P.R. China); Rui Huang (Huazhong University of Science and Technology, P.R. China) pp. 841-844

Fast Facial Landmark Detection Using Cascade Classifiers and a Simple 3D Model Ang Liu (Peking University, P.R. China); Yangzhou Du (Intel China Research Center, P.R. China); Tao Wang (Intel China Research Center, P.R. China); Jianguo Li (Intel China Research Center, Beijing, P.R. China); Eric Li (Intel China Research Centre, Intel Corp., P.R. China); Yimin Zhang (Intel China Research Center, Canada); Yong Zhao (Peking University, P.R. China) pp. 845-848 


\section{MP.PB: 3D Modeling and Synthesis (Poster)}

Intrinsic Geometric Distortions in a Type of Multi-Projector Light Field Display Amir Said (Hewlett Packard Laboratories, USA) pp. 849-852

\section{Optimal Conditions for Camera Calibration Using a Planar Template} Carlos Ricolfe-Viala (Universidad Politecnica Valencia, Spain); Antonio-Jose Sanchez-Salmeron (Universidad Politecnica Valencia, Spain) pp. 853-856

Zhang's One-Dimensional Calibration Revisited with the Heteroscedastic Error-InVariables Model Liang Wang (Beijing University of Technology, P.R. China) pp. 857-860

\section{Blind Correction of Lens Aberration Using Zernike Moments} Kambiz Rahbar (Young Researchers Club, Islamic Azad University, Tehran Center, Iran); Karim Faez (Amirkabir University of Technology, Iran) pp. 861-864

Semi-Automatic 2D to 3D Image Conversion Using Scale-Space Random Walks and a Graph Cuts Based Depth Prior

Raymond Phan (Ryerson University, Canada); Richard J Rzeszutek (Ryerson University, Canada); Dimitri Androutsos (Ryerson University, Canada) pp. 865-868

\section{Dense Interpolation of 3D Points Based on Surface and Color} Zhaoyin Jia (Cornell University, USA); Yao-Jen Chang (Cornell University, USA); Tzung-Han Lin (Industrial Technology Research Institute, Taiwan); Tsuhan Chen (Cornell University, USA) pp. 869-872

\section{Robust Albedo Estimation From a Facial Image with Cast Shadow} Sungho Suh (Seoul National University, Korea); Minsik Lee (Seoul National University, Korea); Chong-Ho Choi (Seoul National University, Korea) pp. 873-876

Generating Compact Meshes Under Planar Constraints: An Automatic Approach for Modeling Buildings From Aerial LiDAR Yannick Verdié (INRIA, France); Florent Lafarge (INRIA, France); Josiane Zerubia (INRIA, Sophia Antipolis, France) pp. $877-880$

Patch-Sweeping with Robust Prior for High Precision Depth Estimation in Real-Time Systems

Wolfgang Waizenegger (Fraunhofer Heinrich-Hertz-Institut, Germany); Nicole Atzpadin (Fraunhofer Heinrich-Hertz-Institut, Germany); Oliver Schreer (Fraunhofer Heinrich-Hertz-Institut, Germany); Ingo Feldmann (Fraunhofer Heinrich-Hertz-Institut, Germany) pp. 881-884 
Marker-less Human Pose Estimation and Surface Reconstruction Using a Segmented Model

Weilan Luo (University of Tokyo, Japan); Toshihiko Yamasaki (The University of Tokyo, Japan); Kiyoharu Aizawa (University of Tokyo, Japan)

pp. 885-888

\section{A Dynamic Approach for Approximate Pairwise Alignment Based on 4-Points}

Congruence Sets of $3 D$ Points

Juarez Silva Junior (University of Brasilia, Brazil); Dibio L Borges (University of Brasilia, Brazil); Flavio Vidal (University of Brasilia, Brazil)

pp. 889-892

Implicit B-Spline Fitting Using the 3L Algorithm

Mohammad Rouhani (Computer Vision Center, Spain); Angel D. Sappa (Computer

Vision Center, Spain)

pp. 893-896

Surface Completion of Shape and Texture Based on Energy Minimization Norihiko Kawai (University of California at Berkeley, USA); Avideh Zakhor (University of California at Berkeley, USA); Tomokazu Sato (Nara Institute of Science and Technology, Japan); Naokazu Yokoya (Nara Institute of Science and Technology, Japan) pp. 897-900

Dense Point-To-Point Correspondences Between 3D Faces with Large Variations for Constructing 3D Morphable Models

Moritz Kaiser (Technical University of Munich, Germany); Nicolas H. Lehment (Technische Universität München, Germany); Gerhard Rigoll (Technische Universität München, Germany)

pp. 901-904

3D Mesh Compression Based on Dual-Ring Prediction and MMSE Prediction Dae-Youn Lee (Korea University, Korea); Jae-Kyun Ahn (Korea University, Korea); Minsu Ahn (Samsung Advanced Institute of Technology, Korea); James D. K. Kim (Samsung Advanced Institute of Technology, Korea); Changyeong Kim (Samsung Advanced Institute of Technology, Korea); Chang-Su Kim (Korea University, Korea) pp. 905-908

Visual Pertinent 2D-to-3D Video Conversion by Multi-cue Fusion Zhebin Zhang (Institute of Computing Technologies, Chinese Academy of Science, P.R. China); Yizhou Wang (Peking University, P.R. China); Tingting Jiang (Peking University, P.R. China); Wen Gao (Peking University, P.R. China) pp. 909-912

MP.PC: Image \& Video Communication (Poster)

\section{QoE-driven Resource Optimization for User Generated Video Content in Next Generation Mobile Networks}

Ali El Essaili (Munich University of Technology, Germany); Eckehard Steinbach (Munich University of Technology, Germany); Daniele Munaretto (University of Padova, Italy); Srisakul Thakolsri (DoCoMo Euro-Labs, Germany); Wolfgang Kellerer (DOCOMO Communications Laboratories Europe, Germany) 
pp. 913-916

\section{Secure Transcoding for Compressive Multimedia Sensing}

Li-Wei Kang (Academia Sinica, Taiwan); Chih-Yang Lin (Asia University, Taiwan);

Hung-Wei Chen (Academia Sinica, Taiwan); Chia-Mu Yu (Academia Sinica and Natioanl Taiwan University, Taiwan); Chun-Shien Lu (Institute of Information Science, Academia Sinica, Taiwan); Chao-yung Hsu (Academia Sinica, Taiwan); Soo-Chang Pei (National Taiwan University, Taiwan) pp. $917-920$

\section{Scalable Compressive Video}

Vladimir Stankovic (University of Strathclyde, United Kingdom); Lina Stankovic (University of Strathclyde, United Kingdom); Samuel Cheng (University of Oklahoma, USA)

pp. 921-924

Synchronization of Presentation Slides and Lecture Videos Using Bit Rate Sequences Georg Schroth (Technische Universität München, Germany); Ngai-Man Cheung (Stanford University, USA); Eckehard Steinbach (Munich University of Technology, Germany); Bernd Girod (Stanford University, USA) pp. 925-928

Effect of a Synthesized Depth View on Multi-View Rendering Quality Jin Young Lee (Samsung Electronics Co., Ltd., Korea); Jaejoon Lee (Samsung Electronics, Korea); Dusik Park (Advanced Media Lab, SAIT, Samsung Electronics, Korea) pp. 929-932

Capacity Improvement in eMBMS Using SVC and Layer-Aware Bearer Allocation Cornelius Hellge (Fraunhofer Institute for Telecommunications - Heinrich-HertzInstitute, Germany); Robert Skupin (Technische Universität Berlin \& Fraunhofer Institute for Telecommunications, Heinrich-Hertz-Institut, Germany); Jaihyung Cho (ETRI, Korea); Thomas Schierl (Fraunhofer HHI, Germany); Thomas Wiegand (Fraunhofer Institute for Telecommunications - Heinrich-Hertz-Institute, Germany) pp. 933-936

\section{Robust Video Transmission Using Pyramid Vector Quantisation}

Syed Mohsin Matloob Bokhari (University of Bristol, United Kingdom); David Bull (University of Bristol, United Kingdom); Andrew Nix (University of Bristol, United Kingdom) pp. 937-940

An Improved Cross-layer Mapping Mechanism for Packet Video Delivery Over Wlan Haidong Wang (Xi'an Jiaotong University, P.R. China); Guizhong Liu (Xi'an Jiaotong University, P.R. China); Qinli Wang (Xi'an Jiaotong University, P.R. China) pp. 941-944

\section{Fairness and QoS Guaranteed User Scheduling for Multi-user MIMO Broadcasting} Channel

Qian Liu (University at Buffalo, USA); Chang Wen Chen (State University of New York at Buffalo, USA)

pp. $945-948$

Performance of H.264 with Isolated Bit Error: Packet Decode or Discard? Murat Demirtas (University of California, Irvine, USA); Amy Reibman (AT\&T Labs Research, USA); Hamid Jafarkhani (University of California, Irvine, USA) 
pp. 949-952

Efficient Iterative Receiver for LDPC Coded Wireless IPTV System

YouZhe Fan (The Hong Kong University of Science and Technology, Hong Kong); James She (University of Cambridge, United Kingdom); Cy Tsui (HKUST, Hong Kong) pp. 953-956

High Performance H.264/AVC Encoding Motion Prediction Algorithm Ronaldo Husemann (UNIVATES, Brazil); Valter Roesler (Federal University of Rio Grande do Sul (UFRGS), Brazil); Altamiro A Susin (Federal University of Rio Grande do Sul, Brazil)

pp. 957-960

Spatial Prediction Based on Self-Similarity Compensation for 3D Holoscopic Image and Video Coding

Caroline Conti (Instituto de Telecomunicacoes, Portugal); João Lino (Instituto de Telecomunicações, Portugal); Paulo Nunes (ISCTE-IUL / Instituto de Telecomunicações, Portugal); Luis Ducla Soares (I.S.C.T.E. / I.T. - Lisbon, Portugal); Paulo Lobato Correia (Instituto Superior Tecnico - Universidade Tecnica Lisboa \& Instituto de Telecomunicacoes, Portugal)

pp. 961-964

\section{MP.PD: Stereo Processing (Poster)}

\section{Robust Color Correction in Stereo Vision}

Qi Wang (Chinese Academy of Sciences, P.R. China); Pingkun Yan (Chinese Academy of Sciences, P.R. China); Yuan Yuan (Chinese Academy of Sciences, P.R. China); Xuelong Li (Xi'an Institute of Optics and Precision Mechanics, Chinese Academy of Sciences, P.R. China)

pp. $965-968$

\section{Accurate Depth Estimation Using Structured Light and Passive Stereo Disparity}

\section{Estimation}

Qiang Li (UNSW@ADFA, Australia); Moyuresh Biswas (University of New South Wales \& Australian Defence Force Academy, Australia); Mark Pickering (UNSW@adfa, Australia); Michael R Frater (The University of New South Wales, Australia)

pp. 969-972

\section{Cryo-Balloon Reconstruction From Two Views}

Andreas Kleinoeder (Friedrich-Alexander-University Erlangen-Nuremberg, Germany); Alexander Brost (Pattern Recognition Lab, Germany); Felix Bourier (Klinik fuer Herzrhythmusstoerungen, Germany); Martin Koch (Pattern Recognition Lab, Germany); Klaus Kurzidim (Klinik fuer Herzrhythmusstoerungen, Germany); Joachim Hornegger (University of Erlangen-Nuremberg, Germany); Norbert Strobel (Siemens AG, Germany)

pp. $973-976$

\section{Image Rectification for Single Camera Stereo System}

Lingfeng Xu (HKUST, Hong Kong); Oscar C. Au (HKUST, Hong Kong); Wenxiu Sun (HKUST, Hong Kong); Yujun Li (Hong Kong University of Science and Technology, Hong Kong); Sung Him Chui (HKUST, Hong Kong); Chun Wing Kwok (HKUST, Hong Kong) 
pp. $977-980$

Stereoscopic Vision Through Epipolarization Without Orientation Parameters José Herraéz (Politecnic University of Valencia, Spain); José Luis Denia (Politechnic University of Valencia, Spain); Pablo Navarro (Politechnic University of Valencia, Spain); Jaime Rodriguez (University of Santiago de Compostela, Spain); María Teresa Martin (University of Santiago de Compostela, Spain) pp. 981-984

Assisting System of Visually Impaired in Touch Panel Operation Using Stereo Camera Atsushi Yamashita (Shizuoka University, Japan); So Kuno (Shizuoka University, Japan); Toru Kaneko (Shizuoka University, Japan) pp. $985-988$

\section{Effect of Brightness on the Quality of Visual 3D Perception}

Mahsa T. Pourazad (TELUS Communications Company, Canada); Zicong Mai (University of British Columbia, Canada); Panos Nasiopoulos (University of British Columbia, Canada); Konstantinos N Plataniotis (University of Toronto, Canada); Rabab Ward (University of British Columbia, Canada) pp. 989-992

\section{Exploiting Spatial Consistency for Object Classification and Pose Estimation} Michael Hödlmoser (Vienna University of Technology, Austria); Branislav Micusik (AIT Austrian Institute of Technology, Austria); Martin Kampel (Vienna University of Technology, Austria) pp. 993-996

Joint Multi-view Foreground Segmentation and 3D Reconstruction with Tolerance Loop

Jaime Gallego (Technical University of Catalonia (UPC), Spain); Jordi Salvador (Technical University of Catalonia (UPC), Spain); Josep R. Casas (Technical University of Catalonia \& UPC BarcelonaTech, Spain); Montse Pardàs (Universitat Politècnica de Catalunya (UPC), Spain) pp. $997-1000$

Dynamic Voxel Carving in Tennis Based on Player Localisation Using a Low Cost Camera Network

David S Monaghan (Dublin City University (DCU), Ireland); Philip T Kelly (Dublin City University, Ireland); Noel. E. O'Connor (Dublin City University, Ireland) pp. 1001-1004

\section{A Convex-Optimization Approach to Dense Stereo Matching}

Yujun Li (Hong Kong University of Science and Technology, Hong Kong); Oscar C. Au (HKUST, Hong Kong); Lingfeng Xu (HKUST, Hong Kong); Wenxiu Sun (HKUST, Hong Kong); Sung Him Chui (HKUST, Hong Kong); Chun Wing Kwok (HKUST, Hong Kong) pp. 1005-1008

Two-View Geometry Estimation Using the Rodrigues Rotation Formula Lorenzo Sorgi (Via Maiorise \& CIRA, Italy) pp. 1009-1012

\section{A New Fast Motion Estimation and Mode Decision Algorithm for H.264 Depth Maps Encoding in Free Viewpoint TV \\ Gianluca Cernigliaro (Universidad Politécnica de Madrid, Spain); Matteo Naccari (British Broadcasting Corporation - Research and Development, United Kingdom); Fernando Jaureguizar (Universidad Politécnica de Madrid, Spain); Julián Cabrera}


(Universidad Politécnica de Madrid, Spain); Fernando Pereira (IST-IT, Portugal); Narciso García (Universidad Politécnica de Madrid, Spain)

pp. 1013-1016

\section{MP.PE: Features for Image Analysis (Poster)}

\section{Bounded Multivariate Surfaces on Monovariate Internal Functions}

Shriprakash Sinha (Tu Delft, The Netherlands); Gert J. ter Horst (UMCG,

Neuroimaging Center, The Netherlands)

pp. 1017-1020

\section{A Fast Component-Tree Algorithm for High Dynamic-Range Images and Second Generation Connectivity}

Michael H.F. Wilkinson (University of Groningen, The Netherlands)

pp. 1021-1024

\section{Corner Detection on Hexagonal Pixel Based Images}

Si Jing Liu (Hong Kong University of Science and Technology, Hong Kong); Sonya A Coleman (University of Ulster, United Kingdom); Dermot Kerr (University of Ulster, United Kingdom); Bryan W. Scotney (University of Ulster, United Kingdom); Bryan Gardiner (University of Ulster, United Kingdom) pp. 1025-1028

\section{A Novel Region-Based Active Contour Approach Relying on Local and Global} Information

Wassima Aitfares (LAAS-CNRS, Université Paul Sabatier, France); Ariane Herbulot (LAAS-CNRS \& University of Toulouse, France); Michel Devy (LAAS-CNRS, France); Houssine Bouyakhf (Université Mohammed V Agdal, Morocco); Fakhita Regragui (Mohammed V Agdal University, Morocco) pp. 1029-1032

\section{A Novel Feature Descriptor Based on the Shearlet Transform} William Robson Schwartz (University of Campinas, Brazil); Ricardo Dutra da Silva (University of Campinas, Brazil); Larry S Davis (University of Maryland, USA); Helio Pedrini (Institute of Computing, University of Campinas, Brazil) pp. 1033-1036

\section{A Robust Parametric Active Contour Based on Fourier Descriptors} Tao Li (INRIA Rennes-Bretagne Atlantique \& EPI LAGADIC, France); Alexandre Krupa (INRIA Rennes-Bretagne Atlantique, France); Christophe Collewet (INRIA Rennes-Bretagne Atlantique, France) pp. $1037-1040$

\section{A DAISY-like Compass Operator}

Xiaojin Gong (Zhejiang University, P.R. China); Jilin Liu (Zhejiang University, P.R. China) pp. 1041-1044

\section{Ellipse Detection Using Sampling Constraints}

Yi Tang (University at Buffalo, The State University of New York \& Center of Excellence for Document Analysis and Recognition, USA); Sargur Srihari (Cedar Buffalo, USA)

pp. $1045-1048$ 


\section{A Shape Contour Descriptor Based on Salience Points}

Glauco Pedrosa (Federal University of Uberlândia, Brazil); Celia Barcelos (Federal University of Uberlândia, Brazil); Marcos Batista (Federal University of Goiás, Brazil) pp. 1049-1052

Boosting Global Scene Classification Accuracy by Discriminative Region Localization Thanh Duc Ngo (The Graduate University for Advanced Studies, Japan); Duy-Dinh Le (National Institute of Informatics, Japan); Shin'ichi Satoh (National Institute of Informatics, Japan) pp. 1053-1056

Color-based Lips Extraction Applied to Voice Activity Detection Carlos Lopes (UFRGS, Brazil); Andre Gonçalves (UFRGS, Brazil); Jacob Scharcanski (UFRGS, Brazil); Claudio R Jung (Universidade Federal do Rio Grande do Sul, Brazil)

pp. 1057-1060

High-performance ASIC Architecture for Hysteresis Thresholding and Component Feature Extraction in Limited-Resource Applications

Mayssaa Al Najjar (University of Louisiana at Lafayette, USA); Swetha Karlapudi (University of Louisiana at Lafayette, USA); Magdy Bayoumi (University of Louisiana, USA) pp. 1061-1064

Application of Complex Networks for Automatic Classification of Damaging Agents in Soybean Leaflets

Thiago Souza (Universidade Federal de Ouro Preto, Brazil); Eduardo Mapa (Prefeitura Municipal de Ouro Preto, Brazil); Kayran Santos (Universidade Federal de Ouro Preto, Brazil); David Menotti (Universidade Federal de Ouro Preto, Brazil) pp. $1065-1068$

\section{Free-Form Anisotropy: A New Method for Crack Detection on Pavement Surface} Images

Tien Sy Nguyen (Vectra, France); Stéphane Begot (University Orleans, France); Florent Duculty (PRISME, University of Orleans, France); Manuel Avila (University of Orleans \& PRISME Laboratory, France) pp. 1069-1072

\section{Efficient Quantization of Color Sift for Image Classification}

Xiao Zhou (Hefei Normal University, P.R. China); Cai-Zhi Zhu (National Institute of Informatics, Japan); Shin'ichi Satoh (National Institute of Informatics, Japan) pp. 1073-1076

\section{MP.PF: Image \& Video Synthesis (Poster)}

Time-Variant Modeling for General Surface Appearance

Yi-Lei Chen (National Tsing Hua University, Taiwan); Chiou-Ting Hsu (National Tsing Hua University, Taiwan)

pp. 1077-1080 
Feature-preserving Thumbnail Generation Based on Graph Cuts

Seong-Gyun Jeong (Korea University, Korea); Chang-Su Kim (Korea University, Korea)

pp. 1081-1084

Synthesis of Two-Dimensional Fractional Brownian Motion Via Circulant Embedding Donny Danudirdjo (The University of Tokyo, Japan); Akira Hirose (The University of Tokyo, Japan)

pp. 1085-1088

\section{Bi-layer Inpainting for Novel View Synthesis}

Hwasup Lim (Samsung Advanced Institute of Technology, Korea); Yong Sun Kim (Samsung Advanced Institute of Technology, Korea); Seungkyu Lee (Samsung Advanced Institute of Technology, Korea); Ouk Choi (Samsung Advanced Institute of Technology, Korea); James D. K. Kim (Samsung Advanced Institute of Technology, Korea); Changyeong Kim (Samsung Advanced Institute of Technology, Korea) pp. 1089-1092

\section{Augmented Reality Mirror for Virtual Facial Alterations}

Vlado Kitanovski (Queen Mary, University of London \& Multimedia and Vision Lab, United Kingdom); Ebroul Izquierdo (Queen Mary, University of London, United Kingdom) pp. 1093-1096

\section{D/3D Virtual Face Modeling}

SoonKee Chung (Korea Advanced Institute of Science and Technology, USA); JeanCharles Bazin (KAIST, Korea); In-So Kweon (Korea Advanced Institute of Science and Technology (KAIST), Korea)

pp. $1097-1100$

\section{Synthesizing for Face Recognition}

Yuelong Li (Peking University, P.R. China); Jufu Feng (Peking University, P.R. China) pp. 1101-1104

\section{Efficient Rendering Distortion Estimation for Depth Map Compression}

Quwen Zhang (Shanghai University \& School of Communication and Information Engineering, P.R. China); Ping An (School of Communication and Information Engineering, ShanghaiUniversity, P.R. China) pp. $1105-1108$

On the Use of Hemispherical Harmonics for Modeling Images of Objects Under Unknown Distant Illumination

Shireen Elhabian (University of Louisville, USA); Ham Rara (University of Louisville, USA); Aly Farag (University of Louisville, USA) pp. 1109-1112

Aesthetic Enhancement of Landscape Photographs as Informed by Paintings Across Depth Layers

Xiaoyan Zhang (Nanyang Technological University, Singapore); Martin Constable (Nanyang Technological University, Singapore); Kap Chan (NTU, Singapore) pp. 1113-1116

Virtual Ads Insertion in Street Building Views for Augmented Reality Yu Huang (Huawei Technologies, USA); Qiang Hao (Western Virginia University, USA); Heather Yu (Huawei Technologies (USA), USA) 
pp. $1117-1120$

Color Style Transfer by Constraint Locally Linear Embedding

Kun Zeng (Sun Yat-Sen University, P.R. China); Liang Lin (Sun Yat-Sen University, P.R. China)

pp. 1121-1124

Face Sketch-Photo Synthesis Based on Support Vector Regression

Jiewei Zhang (Xidian University, P.R. China); Nannan Wang (Xidian University, P.R. China); Xinbo Gao (Xidian University, P.R. China); Dacheng Tao (University of

Technology, Sydney, Singapore); Xuelong Li (Xi'an Institute of Optics and Precision Mechanics, Chinese Academy of Sciences, P.R. China)

pp. $1125-1128$

Efficient Face Hallucination by Using Shape and Texture Dependency

Aydin Akyol (Istanbul Technical University, Turkey); Muhittin Gökmen (Istanbul Technical University \& Faculty of Computer and Informatics, Turkey) pp. 1129-1132

Adaptive KPCA-Based Missing Texture Reconstruction Approach Including Classification Scheme Via Difference Subspaces

Takahiro Ogawa (Hokkaido University, Japan); Miki Haseyama (Hokkaido University, Japan)

pp. $1133-1136$

\section{MP.PG: Interpolation, Resizing and Super-resolution (Poster)}

Isotropic Huber MRFs for Structure Super-Resolution

Youngjin Park (Seoul National University, Korea); Suk Yoo (Seoul National University, Korea)

pp. $1137-1140$

Single Image Super Resolution with High Resolution Dictionary

Guangwu Mu (Xidian University, P.R. China); Xinbo Gao (Xidian University, P.R.

China); Kaibing Zhang (Xidian University, P.R. China); Xuelong Li (Xi'an Institute of Optics and Precision Mechanics, Chinese Academy of Sciences, P.R. China); Dacheng Tao (University of Technology, Sydney, Singapore) pp. 1141-1144

Super-Resolution Interpolation with a Quasi Blur-Hypothesis

Takahiro Saito (Kanagawa University, Japan); Ken-ichi Ishikawa (Kanagawa University, Japan); Takashi Komatsu (Kanagawa University, Japan) pp. $1145-1148$

Adaptive Incremental Video Super-Resolution with Temporal Consistency Heng Su (Tsinghua University, P.R. China); Ying Wu (Northwestern University, USA); Jie Zhou (Tsinghua University, P.R. China) pp. 1149-1152

\section{Superfast Superresolution}

Filip Šroubek (Institute of Information Theory and Automation, Czech Republic); Jan Kamenický (Institute of Information Theory and Automation, Czech Republic);

Peyman Milanfar (University of California, Santa Cruz, USA)

pp. 1153-1156 
Video Coding with Wavelet Image Size Reduction and Wavelet Super Resolution Reconstruction

Yasutaka Matsuo (NHK (Japan Broadcasting Corporation), Japan); Toshie Misu (NHK (Japan Broadcasting Corporation), Japan); Shinichi Sakaida (NHK, Japan); Yoshiaki Shishikui (NHK, Japan)

pp. $1157-1160$

Single Image Super Resolution Via Texture Constrained Sparse Representation Haitao Yin (Hunan University, P.R. China); Shutao Li (Hunan University, P.R. China); Jianwen Hu (Hunan University, P.R. China) pp. $1161-1164$

\section{Fast Edge-Filtered Image Upsampling}

Shantanu H Joshi (UCLA \& UCLA, USA); Antonio Marquina (University of Valencia, Spain); Stan Osher (University of California Los Angeles, USA); Ivo Dinov (UCLA, USA); Arthur Toga (UCLA, USA); John Van Horn (UCLA, USA) pp. $1165-1168$

A Structure-guided Conditional Sampling Model for Video Resolution Enhancement Ying Liu (University of Waterloo, Canada); Alexander Wong (University of Waterloo, Canada); Paul Fieguth (University of Waterloo, Canada) pp. 1169-1172

Bayesian Frame Interpolation by Fusing Multiple Motion-compensated Prediction Frames

Hongbin Liu (Harbin Institute of Technology, P.R. China); Ruiqin Xiong (Peking University, P.R. China) pp. 1173-1176

\section{Similarity Modulated Block Estimation for Image Interpolation}

Jie Ren (Peking University, P.R. China); Jiaying Liu (Peking University, P.R. China); Wei Bai (Peking University, P.R. China); Zongming Guo (Peking University, P.R. China) pp. $1177-1180$

\section{Springs-based Simulation for Image Retargeting} Roberto Gallea (Università degli Studi di Palermo, Italy); Edoardo Ardizzone (University of Palermo, Italy); Roberto Pirrone (University of Palermo, Italy) pp. 1181-1184

Fast and High Quality Learning-based Super-Resolution Utilizing TV Regularization Method

Tomio Goto (Nagoya Institute of Technology, Japan); Shotaro Suzuki (Nagoya Institute of Technology, Japan); Satoshi Hirano (Nagoya Institute of Technology, Japan); Masaru Sakurai (Nagoya Insitute of Technology, Japan); Truong Nguyen (University of California in San Diego, USA) pp. 1185-1188

A Hidden Markov Model-based Methodology for Intra-field Video Deinterlacing Amin Behnad (University of Toronto, Canada); Konstantinos N Plataniotis (University of Toronto, Canada); Xiaolin Wu (McMaster University, Canada) pp. $1189-1192$ 
Transform Domain Semi-Super Resolution

Edson Hung (Universidade de Brasilia, Brazil); Diogo Garcia (Universidade de Brasília, Brazil); Ricardo L de Queiroz (University of Brasil, Brazil)

pp. 1193-1196

Efficient Super-Resolution Driven by Saliency Selectivity

Nabil Sadaka (Arizona State University, USA); Lina Karam (Arizona State University, USA)

pp. $1197-1200$

\section{Tuesday, September 13}

\section{TA.L1: Advances in Motion Representation for Video Coding (Special Session)}

A Block-adaptive Skip Mode for Inter Prediction Based on Parametric Motion Models Alexander Glantz (Technische Universität Berlin, Germany); Michael Tok (Technische Universität Berlin, Germany); Andreas Krutz (Technische Universität Berlin, Germany); Thomas Sikora (Technische Universität Berlin, Germany) pp. 1201-1204

Fast Encoding Algorithms for Geometry-Adaptive Block Partitioning

Philippe Bordes (Technicolor, France); Edouard Francois (Technicolor, France);

Dominique Thoreau (Technicolor, France) pp. 1205-1208

break

Efficient Coding of Video Sequences by Non-Local In-Loop Denoising of Reference Frames

Eugen Wige (University of Erlangen-Nuremberg, Germany); Gilbert Yammine (University of Erlangen-Nuremberg, Germany); Peter Amon (Siemens, Germany); Andreas Hutter (Siemens Corporate Technology, Germany); Andre Kaup (University of Erlangen-Nuremberg, Germany)

pp. $1209-1212$

\section{Generalized Interpolation for Motion Compensated Prediction}

Haricharan Lakshman (Fraunhofer HHI, Germany); Heiko Schwarz (Fraunhofer HHI, Germany); Thierry Blu (CUHK, Hong Kong); Thomas Wiegand (HHI/FhG, Germany) pp. 1213-1216

Picture-level Parameteric Motion Representation for Efficient Motion Compensation Jaewon Sung (LG Electronics, Korea); Seung-Wook Park (LG Electronics, Korea); Joonyoung Park (LG Electronics, Korea); Byeong-Moon Jeon (LG Electronics, Korea) pp. $1217-1220$

\section{Bi-prediction Combining Template and Block Motion Compensations}

Chung-Lin Lee (National Chiao Tung University, Taiwan); Chun-Chi Chen (National

Chiao Tung University, Taiwan); Yi-Wen Chen (National Chiao-Tung University, 
Taiwan); Mu-Hsuan Wu (National Chiao Tung University, Taiwan); Chung-Hao Wu (National Chiao Tung University, Taiwan)

pp. 1221-1224

\section{TA.L2: Sparse Representation and Sparse Coding (Lecture)}

\section{Learning Complex Image Patterns with Scale and Shift Invariant Sparse Coding} Xiaobing Liu (Tsinghua University, P.R. China); Bo Zhang (Tsinghua University, P.R. China)

pp. $1225-1228$

On Sparse Representations of Color Images

Xiaolin Wu (McMaster University, Canada); Guangtao Zhai (McMasster University, Canada)

pp. 1229-1232

break

Hyperspectral Image Classification Via Kernel Sparse Representation

Yi Chen (Johns Hopkins University, USA); Nasser Nasrabadi (US Army Research Laboratory, USA); Trac D. Tran (Johns Hopkins University, USA) pp. $1233-1236$

Improved Sparse Coding Using Manifold Projections Karthikeyan Natesan Ramamurthy (Arizona State University, USA); Jayaraman Thiagarajan (Arizona State University, USA); Andreas Spanias (ASU / SenSIP Center / School of ECEE, USA) pp. $1237-1240$

\section{Learning Invariant Color Features with Sparse Topographic Restricted Boltzmann}

\section{Machines}

Hanlin Goh (Institute for Infocomm Research \& Université Pierre et Marie Curie, Singapore); Łukasz Kuśmierz (Institute for Infocomm Research, Singapore); JooHwee Lim (Institute for Infocomm Research, Singapore); Nicolas Thome (University Pierre et Marie Curie, France); Matthieu Cord (UPMC Paris 6, France) pp. 1241-1244

\section{Learning Dictionary Via Subspace Segmentation for Sparse Representation} Jianzhou Feng (Shanghai Jiao Tong University, P.R. China); Li Song (Shanghai Jiao Tong University, P.R. China); Xiaokang Yang (Shanghai Jiao Tong University, P.R. China); WenJun Zhang (Shanghai JiaoTong University, P.R. China) pp. $1245-1248$

\section{TA.L3: Image Classification (Lecture)}

\section{CW-SSIM Based Image Classification}

Yang Gao (University of Waterloo, Canada); Abdul Rehman (University of Waterloo, Canada); Zhou Wang (University of Waterloo, Canada)

pp. 1249-1252 
Tree Trunk Detection Using Contrast Templates

Yan Lu (University of Delaware, USA); Christopher Rasmussen (University of Delaware, USA)

pp. $1253-1256$

break

Human Skin Detection in Images by MSER Analysis

Lei Huang (Institute of Computing Technology, Chinese Academy of Sciences \& Graduate University of Chinese Academy of Sciences, P.R. China); Tian Xia (Institute of Computing Technology, Chinese Academy of Sciences, P.R. China); Yongdong Zhang (Institute of Computing Technology, Chinese Academy of Sciences, P.R. China); Shou-Xun Lin (Institute of Computing Technology, Chinese Academy of Sciences, P.R. China) pp. $1257-1260$

HMAX-S: Deep Scale Representation for Biologically Inspired Image Categorization Christian Theriault (Pierre et Marie Currie University, France); Nicolas Thome (University Pierre et Marie Curie, France); Matthieu Cord (UPMC Paris 6, France) pp. 1261-1264

Stochastic Minimum Spanning Forest Approach for Spectral-Spatial Classification of Hyperspectral Images

Kévin Bernard (University of Iceland \& Heriot-Watt University, United Kingdom); Yuliya Tarabalka (GIPSA-Lab, France); Jesus Angulo (MINES Paristech, France); Jocelyn Chanussot (Grenoble Institute of Technology, France); Jon Benediktsson (University of Iceland, Iceland) pp. $1265-1268$

TA.L4: Image Analysis Using Multi-Scale and Hierarchical Methods (Lecture)

Optimized Butterfly-based Lifting Scheme for Semi-Regular Meshes Aymen Kammoun (I3S-CNRS-University of Nice Sophia Antipolis, France); Frédéric Payan (I3S-CNRS-University of Nice Sophia Antipolis, France); Marc Antonini (I3SCNRS-University of Nice Sophia Antipolis, France) pp. 1269-1272

\section{Hyperspectral Image Segmentation Using Binary Partition Trees}

Silvia Valero (Universitat Politecnica de Catalunya, Spain); Philippe Salembier (Universitat Politecnica de Catalunya, Spain); Jocelyn Chanussot (Grenoble Institute of Technology, France) pp. $1273-1276$

break

Testing a Multivariate Model for Wavelet Coefficients

Roland Kwitt (University of Salzburg, Austria); Peter Meerwald (University of Salzburg, Austria); Andreas Uhl (Salzburg University, Austria); Geert Verdoolaege (Ghent University, Belgium)

pp. $1277-1280$ 
3D Facial Expression Analysis by Using 2D and 3D Wavelet Transforms Sílvia Cristina Dias Pinto (University of Sao Paulo, Brazil); Jesús P. Mena-Chalco (University of Sao Paulo \& Institute of Mathematics and Statistics, Brazil); Fabrício Martins Lopes (Federal University of Technology - Parana \& UTFPR, Brazil); Luiz Velho (IMPA, Brazil); Roberto Cesar Junior (University of São Paulo, Brazil) pp. 1281-1284

Detecting Gestures in Medieval Images Joseph Schlecht (University of Heidelberg, Germany); Bernd Carqué (University of Heidelberg, Germany); Björn Ommer (IWR - University of Heidelberg, Germany) pp. $1285-1288$

Pose Estimation and Body Segmentation Based on Hierarchical Searching Tree Li Shifeng (Dalian University of Technology, P.R. China); Huchuan Lu (Dalian University of Technology, P.R. China); Ruan (OMRON Corp., Japan); Yen-Wei Chen (Ritsumeikan University, Japan) pp. 1289-1292

TA.L5: Astronomy and Cosmology (Special Session)

Bayesian MAP Detection of Extragalactic Point Sources in Microwave Astronomical Images

Diego Herranz (Instituto de Fisica de Cantabria, Santander, Spain); Francisco

Argüeso (Universidad de Oviedo, Spain); Emanuele Salerno (ISTI-CNR, Italy); Ercan Kuruoğlu (CNR, Pisa, Italy); Koray Kayabol (INRIA, France) pp. 1293-1296

Source Separation in Cosmology, From Global to Local Models Jerome Bobin (CEA, France); Florent Sureau (CEA, France); Jean-Luc Starck (CEA, France)

pp. 1297-1300

break

Data Augmentation for Galaxy Density Map Reconstruction Francois-Xavier Dupé (CEA, France); Jalal Fadili (GREYC CNRS UMR 6072, ensicaen, France); Jean-Luc Starck (CEA, France) pp. 1301-1304

Image Processing Challenges in Weak Gravitational Lensing Adam Amara (ETHZ, Switzerland) pp. 1305-1308

Scale Invariant Images in Astronomy Through the Lens of Multifractal Modeling Pierre Chainais (INRIA Lille-Nord Europe \& Clermont University, France); Véronique Delouille (Royal Observatory of Belgium, Belgium); Jean-François Hochedez (LATMOS UMR CNRS 8190, France) pp. $1309-1312$

Compressed Sensing for Radio Interferometric Imaging: Review and Future Direction Jason McEwen (Ecole Polytechnique Federale de Lausanne, Switzerland); Yves Wiaux (EPFL, Switzerland) pp. $1313-1316$ 


\section{TA.L6: High Dynamic Range Imaging and Compression (Lecture)}

\section{Spatially Adaptive Filtering for Registration Artifact Removal in HDR Video}

Stephen Mangiat (University of California, Santa Barbara, USA); Jerry D Gibson

(University of California, Santa Barbara, USA)

pp. 1317-1320

Perception-Based High Dynamic Range Video Compression with Optimal Bit-depth Transformation

Yang Zhang (University of Bristol, United Kingdom); Erik Reinhard (University of Bristol, United Kingdom); David Bull (University of Bristol, United Kingdom) pp. $1321-1324$

break

\section{A Single Algorithm Combining Exposure and Focus Fusion} Azhar Sufi (SRI International \& Rutgers University, USA); David C. Zhang (SRI International Sarnoff, USA); Gooitzen van der Wal (SRI International, USA) pp. $1325-1328$

\section{SDALA: Simultaneous Dynamic Range Compression and Local Contrast}

\section{Enhancement Algorithm}

Chi-Yi Tsai (Tamkang University, Taiwan)

pp. 1329-1332

\section{High Dynamic Range Imaging Under Noisy Observations}

Renu Rameshan (Indian Institute of Technology Bombay \& Amrita Vishwa Vidyapeetham, India); Subhasis Chaudhuri (Indian Institute of Technology, Bombay, India); Rajbabu Velmurugan (IIT Bombay, India) pp. $1333-1336$

De-ghosting of HDR Images with Double-credit Intensity Mapping Zijian Zhu (Institute for Infocomm Research, Singapore); Zhengguo Li (Institute for Inforcomm Research, Singapore); Susanto Rahardja (Institute for Infocomm Research, Singapore); Pasi Fränti (University of Eastern Finland, Finland) pp. $1337-1340$

\section{TA.L7: Interpolation and Super-resolution (Lecture)}

\section{Fast Video Interpolation/Upsampling Using Linear Motion Model Kwok-Wai Hung (The Hong Kong Polytechnic University, Hong Kong); Wan-Chi Siu (The Hong Kong Polytechnic University, Hong Kong) pp. 1341-1344}

\section{Parameter Estimation in Bayesian Super-Resolution Pansharpening Using Contourlets}

Israa Amro (Al-Quds Open University, Palestine); Javier Mateos (University of Granada, Spain); Mlguel Vega (University of Granada, Spain) pp. $1345-1348$

break 
Learning Context-Aware Sparse Representation for Single Image Super-Resolution Min-Chun Yang (National Taiwan University, Taiwan); Chang-Heng Wang (National Taiwan University, Taiwan); Ting-Yao Hu (National Taiwan University, Taiwan); YuChiang Frank Wang (Academia Sinica, Taiwan) pp. 1349-1352

Multi-scale Non-Local Kernel Regression for Super Resolution Haichao Zhang (Northwestern Polytechnical University \& University of Illinois at Urbana-Champaign, USA); Jianchao Yang (UIUC, USA); Yanning Zhang (Northwestern Polytechnical University, P.R. China); Thomas S Huang (University of Illinois at Urbana-Champaign, USA) pp. 1353-1356

Two-step Super-resolution Technique Using Bounded Total Variation and Bisquare Mestimator Under Local Illumination Changes

Mohamed M. Fouad (Military Technical College, Egypt); Richard Dansereau (Carleton University, Canada); Anthony Whitehead (Carleton Universty, Canada) pp. 1357-1360

Discrete Infinity Harmonic Functions: Towards a Unified Interpolation Framework on Graphs

Mahmoud Ghoniem (Université de Caen Basse-Normandie, France); Abderrahim Elmoataz (Université de Caen Basse-Normandi, France); Olivier Lezoray (Université de Caen Basse-Normandie \& Greyc UMR CNRS 6072, France) pp. $1361-1364$

\section{TA.L8: Tomographic Imaging (Lecture)}

\section{On the Efficiency of Proximal Methods in CBCT and PET}

Sandrine Anthoine (Université Aix-Marseille, LATP, CNRS, France); Jean Francois Aujol (Université Bordeaux 1, IMB, France); Yannick Boursier (Aix Marseille University \& CPPM, CNRS/IN2P3, France); Clothilde Mélot (Aix Marseille University, LATP / CNRS, France) pp. 1365-1368

Regularized Polychromatic Reconstruction for Transmission Tomography Kwang Eun Jang (Samsung Advanced Institute of Technology, Korea) pp. 1369-1372

break

A Discrete-Continuous Bayesian Model for Emission Tomography Mame Diarra Fall (Université Paris-Sud 11, France); Eric Barat (CEA, France); Claude Comtat (Service Hospitalier Frédéric Joliot, France); Thomas Dautremer (CEA, France); Thierry Montagu (CEA, France); Ali Mohammad-Djafari (Centre national de la recherche scientifique (CNRS), France) pp. 1373-1376

Bayesian Data Fusion and Inversion in X-ray Multi-Energy Computed Tomography Caifang Cai (Commissariat a Energie Atomique, France); Ali Mohammad-Djafari (Centre national de la recherche scientifique (CNRS), France); Samuel Legoupil 
(Commissariat a Energie Atomique (CEA), France); Thomas Rodet (University of Paris Sud \& L2S - UMR 8506 Supelec, CNRS, University Paris Sud, France) pp. $1377-1380$

Improved Kernel-based Limited-View CT Reconstruction Via Anisotropic Diffusion Jun Feng (Sichuan University, Chengdu University of Technology, P.R. China); JianZhou Zhang (Sichuan University, P.R. China)

pp. 1381-1384

\title{
TA.PA: Statistical-Model-Based Processing and Tracking (Poster)
}

\author{
Efficient Joint Poisson-Gauss Restoration Using Multi-Frame L2-relaxed-LO Analysis- \\ based Sparsity \\ Elena Gil-Rodrigo (Consejo Superior de Investigaciones Científicas, Spain); Javier \\ Portilla (Consejo Superior de Investigaciones Científicas, Spain); David Miraut \\ (Universidad Rey Juan Carlos, Spain); Ricardo Suarez-Mesa (Universidad Rey Juan \\ Carlos, Spain) \\ pp. 1385-1388
}

Image-based Object Detection Under Varying Illumination in Environments with Specular Surfaces

Werner A. Maier (Technische Universität München, Germany); Michael Eschey (Technische Universität München, Germany); Eckehard Steinbach (Munich University of Technology, Germany)

pp. 1389-1392

Hybrid Parametric-Nonparametric Modeling with Application to Natural Image Upsampling

Guangtao Zhai (McMasster University, Canada); Xiaolin Wu (McMaster University, Canada)

pp. 1393-1396

Batch-incremental Principal Component Analysis with Exact Mean Update Guifang Duan (Ritsumeikan University, Japan); Yen-Wei Chen (Ritsumeikan University, Japan)

pp. $1397-1400$

A Method for Discontinuous Neurite Reconstruction Based on Diffusion Tensor, Hessian Eigenvector, and Diffused Gradient Vector Fields

HeeChang Kim (Universite Paris Descartes \& Institut Pasteur Korea, Korea); Georges Stamon (Universite Paris Lipade-Sip, France); Auguste Genovesio (Institut Pasteur Korea, Korea)

pp. 1401-1404

Boosting Segmentation Results by Contour Relaxation

Alvaro Guevara (Goethe University, Frankfurt, Germany); Christian Conrad (Goethe University, Germany); Rudolf Mester (Goethe University, Frankfurt \& VSI Lab, Germany) pp. 1405-1408 
Improved DCT Coefficient Distribution Modeling for H.264-like Video Coders Based on Block Classification

Nejat Kamaci (Georgia Institute of Technology, USA); Ghassan AIRegib (Georgia Institute of Technology, USA)

pp. 1409-1412

Flexible Trajectory Modeling Using a Mixture of Parametric Motion Fields for Video Surveillance

Jacinto C. Nascimento (Instituto de Sistemas e Robotica, Portugal); Jorge S. Marques (Instituto Superior Técnico \& Instituto de Sistemas e Robotica, Portugal); João Lemos (Instituto Superior Técnico, Portugal)

pp. 1413-1416

Fast Incremental Method for Matrix Completion: An Application to Trajectory

Correction

Ricardo Cabral (Carnegie Mellon University, USA); João Paulo Costeira (I.S.T. -

Technical U. Lisbon / I.S.R. Lisbon, Portugal); Fernando De la Torre (Carnegie Mellon University, USA); Alexandre Bernardino (I.S.T. - Technical U. Lisbon / I.S.R. Lisbon, Portugal)

pp. $1417-1420$

Liver Tumor Detection in CT Images by Adaptive Contrast Enhancement and the EM/ MPM Algorithm

Yu Masuda (University of Ritsumeikan, Japan); Wei Xiong (Institute for Infocomm Research, Singapore)

pp. 1421-1424

RANSAC-LEL: An Optimized Version with Least Entropy Like Estimators Cosimo Distante (CNR, Italy); Giovanni Indiveri (University of Salento, Italy) pp. $1425-1428$

Discriminative Model Selection Using a Modified Bayesian Criterion: Application to Trajectory Modeling Jacinto C. Nascimento (Instituto de Sistemas e Robotica, Portugal); Jorge S. Marques (Instituto Superior Técnico \& Instituto de Sistemas e Robotica, Portugal); Mario A. T. Figueiredo (Instituto Superior Técnico, Portugal) pp. $1429-1432$

Visual Tracking and Dynamic Learning on the Grassmann Manifold with Inference From a Bayesian Framework and State Space Models Zulfiqar Khan (Chalmers University of Technology, Sweden); Irene Y. H. Gu (Chalmers University of Technology, Sweden) pp. $1433-1436$

Bayesian Visual Surveillance: a Model for Detecting and Tracking a Variable Number of Moving Objects

Carlos R. del-Blanco (Universidad Politécnica de Madrid, Spain); Fernando Jaureguizar (Universidad Politécnica de Madrid, Spain); Narciso García (Universidad Politécnica de Madrid, Spain) pp. $1437-1440$ 
Human Identification Using Body Prior and Generalized EMD

Lianyang Ma (Shanghai Jiao Tong University, P.R. China); Xiaokang Yang (Shanghai Jiao Tong University, P.R. China); Yi Xu (Shanghai Jiao Tong University, P.R. China); Jun Zhu (Shanghai Jiao Tong University, P.R. China) pp. 1441-1444

\title{
TA.PB: Filtering (Poster)
}

\author{
Region-based Thresholding Using Component Tree \\ Alexandre Gonçalves Silva (Santa Catarina State University, Brazil) \\ pp. $1445-1448$
}

Interactive Collection of Training Samples From the Max-Tree Structure

Georgios K Ouzounis (European Commission, Joint Research Centre, Italy); Lionel

Gueguen (JRC - European Commission, Italy)

pp. 1449-1452

\section{A Method to Determine the Size of the Structuring Element in Morphological Correction of Non-uniform Illumination Juan Lorenzo-Ginori (Universidad Central Marta Abreu de Las Villas, Cuba) pp. 1453-1456}

Three Kinds of Color Total-Variation Semi-norms and Its Application to Color-Image Denoising

Takahiro Saito (Kanagawa University, Japan); Yousuke Takagaki (Kanagawa University, Japan); Takashi Komatsu (Kanagawa University, Japan) pp. $1457-1460$

Fast Algorithm for Total Variation Minimization Masaru Sakurai (Nagoya Insitute of Technology, Japan); Satoshi Kiriyama (Nagoya Insitute of Technology, Japan); Tomio Goto (Nagoya Institute of Technology, Japan); Satoshi Hirano (Nagoya Institute of Technology, Japan) pp. 1461-1464

Structural-Context-Preserving Image Abstraction by Using Space-Filling Curve Based on Minimum Spanning Tree

Takanori Koga (Tokuyama College of Technology, Japan); Noriaki Suetake (Yamaguchi University, Japan) pp. 1465-1468

\section{Color Space Influence on Mean Shift Filtering}

Ting Li (Institut National des Sciences Appliquées de Lyon, France); Thomas Grenier (CREATIS, CNRS UMR 5220, Inserm U, France); Hugues Benoit-Cattin (CREATIS, CNRS UMR 5515, Inserm U, France)

pp. $1469-1472$

Adaptive Grid Pattern Artifact Reduction in Radiography Imaging Based on the Significant-Signal Bandwidth

Dong Sik Kim (Hankuk University of Foreign Studies, Korea); Sanggyun Lee (DRTECH Co., Korea)

pp. $1473-1476$ 
A New Infrared Image Fusion Method Using Empirical Mode Decomposition and Inpainting

Yu-Qiu Sun (Yangtze University, P.R. China); Min-Sung Koh (Eastern Washington University, USA); Esteban Rodriguez-Marek (Eastern Washington University, USA); Claudio Talarico (Eastern Washington University, USA) pp. $1477-1480$

Improved Optimal Seam Selection Blending for Fast Video Stitching of Videos Captured From Freely Moving Devices

Motaz El-Saban (Microsoft Research - Cairo Innovation Lab, Egypt); Mostafa Izz (Cairo University, Egypt); Ayman Kaheel (Microsoft Research \& Cairo Innovation Lab, Egypt); Mahmoud Refaat (Microsoft Research, Egypt) pp. 1481-1484

Fast and Robust Isotropic Scaling Iterative Closest Point Algorithm Ce Li (Xi'an Jiaotong University, P.R. China); Jianru Xue (Xi'an Jiaotong University, P.R. China); Nanning Zheng (Xi'an Jiaotong University, P.R. China); Shaoyi Du (Xi'an Jiaotong University, P.R. China); Jihua Zhu (Xi'an Jiaotong University, P.R. China); Zhiqiang Tian (Xi'an Jiaotong University, P.R. China) pp. 1485-1488

Fusion of Panchromatic and Multispectral Images Using Multiscale Dual Bilateral Filter Jianwen Hu (Hunan University, P.R. China); Shutao Li (Hunan University, P.R. China) pp. 1489-1492

Video Mosaicing Using a Mutual Information-based Motion Estimation Process Amaury Dame (CNRS, IRISA, France); Eric Marchand (IRISA - INRIA Rennes, France) pp. 1493-1496

\section{Seam Carving in Wavelet Transform Domain}

Kazu Mishiba (Keio University, Japan); Masaaki Ikehara (Keio University, Japan) pp. 1497-1500

Variable Remapping of Images From Very Different Sources Wei Zhang (SAIC, USA); Rama Chellappa (University of Maryland, USA) pp. 1501-1504

TA.PC: Image Processing: Analysis, Modeling, Enhancement (Poster)

Bayesian Stereoscopic Image Resolution Enhancement

Jing Tian (Wuhan University of Science and Technology, P.R. China); Li Chen (Wuhan University of Science and Technology, P.R. China) pp. 1505-1508

Post Processing for Blocking Artifact Reduction Seok Bong Yoo (KAIST, Korea); Kyuha Choi (KAIST, Korea); Jong Beom Ra (KAIST, Korea) pp. $1509-1512$ 


\section{Separable Bilateral Nonlocal Means}

Yong Sun Kim (Samsung Advanced Institute of Technology, Korea); Hwasup Lim (Samsung Advanced Institute of Technology, Korea); Ouk Choi (Samsung Advanced Institute of Technology, Korea); Keechang Lee (Samsung Advanced Institute of Technology, Korea); James D. K. Kim (Samsung Advanced Institute of Technology, Korea); Changyeong Kim (Samsung Advanced Institute of Technology, Korea) pp. 1513-1516

\section{Multi-resolution Missing Data Interpolation in SST Image Series}

Sileye Oumar Ba (Télécom Bretagne, France); Thomas Corpetti (CNRS-LIAMA, P.R. China); Ronan Fablet (Institut Telecom - Telecom Bretagne, France) pp. $1517-1520$

\section{Same Frame Rate IR to Enhance Visible Video Conference Lighting}

Chen Wu (Google Inc., USA); Ramin Samadani (HP Labs, USA); Prabath

Gunawardane (University of California, Santa Cruz, USA) pp. 1521-1524

\section{An Augmented Lagrangian Method for Fast Gradient Vector Flow Computation} Jianfeng Li (Harbin Institute of Technology, P.R. China); Wangmeng Zuo (Harbin Institute of Technology, P.R. China); Xiaofei Zhao (Harbin Institute of Technology, P.R. China); David Zhang (The Hong Kong Polytechnic University, Hong Kong) pp. $1525-1528$

High Frequency Compensated Face Hallucination

So Sasatani (University of Ritsumeikan, Japan) pp. 1529-1532

Detail Preserving Multiple Bit-Depth Image Representation and Coding Takao Jinno (The University of Kitakyushu, Japan); Masahiro Okuda (The University of Kitakyushu, Japan); Nicola Adami (University of Brescia, Italy) pp. $1533-1536$

\section{Recovering Missing Coefficients in DCT-Transformed Images}

Shujun Li (University of Surrey, United Kingdom); Andreas Karrenbauer (University of Konstanz, Germany); Dietmar Saupe (University of Konstanz, Germany); C.-C. Jay Kuo (University of Southern California, USA) pp. $1537-1540$

\section{Wasserstein Regularization of Imaging Problem}

Julien Rabin (École Normale Supérieure de Cachan, France); Gabriel Peyré (CNRS, Université Paris-Dauphine, France)

pp. 1541-1544

An Adaptive Total Variation Method for Speckle Reduction in Medical Ultrasound Imaging

Meriem Hacini (Laboratoire d' Automatique et de Robotique, Université de Constantine, Algeria); Hachouf Fella (Constantine University, Algeria) pp. $1545-1548$

\section{A Monotonic Constrained Regression Framework for Histogram Equalization and} Specification

Lu-Hung Chen (National Chung Hsing University, Taiwan); Yao-Hsiang Yang

(Research Center for Information Technology Innovation, Acadamia Sinica \& National 
Taiwan University, Taiwan); Chu-Song Chen (Institute of Information Science, Academia Sinica, Taiwan)

pp. 1549-1552

\section{Interactive Motion Deblurring Using Light Streaks}

Binh-Son Hua (National University of Singapore, Singapore); Kok-Lim Low (National University of Singapore, Singapore)

pp. 1553-1556

Fusion-based Restoration of the Underwater Images

Codruta Ancuti (Hasselt University, Belgium); Cosmin Ancuti (Hasselt University, Belgium); Tom Haber (Hasselt University, Belgium); Philippe Bekaert (Hasselt University, Belgium) pp. $1557-1560$

\section{Chi-Square Unbiased Risk Estimate for Denoising Magnitude MR Images} Florian Luisier (Harvard University, USA); Patrick Wolfe (Harvard University, USA) pp. 1561-1564

\section{TA.PD: Biomedical Image Processing and Applications (Poster)}

\section{Computer-aided Cataract Detection Using Enhanced Texture Features on Retro- IIlumination Lens Images \\ Xinting Gao (Institure for Infocomm Research, Singapore); Huiqi Li (Institute for Infocomm Research (I2R), Singapore); Joo-Hwee Lim (Institute for Infocomm Research, Singapore); Tien Yin Wong (Singapore Eye Research Centre, Singapore) pp. $1565-1568$}

\section{D Protein-Protein Docking Using Shape Complementarity and Fast Alignment} Apostolos Axenopoulos (University of Thessaly, Department of Computer \& Communication Engineering \& Informatics \& Telematics Institute, Greece); Petros Daras (Informatics \& Telematics Institute, Greece); Georgios Papadopoulos (University of Thessaly - Department of Biochemistry \& Biotechnology, Greece); Elias Houstis (University of Thessaly, Department of Computer \& Communication Engineering, Greece) pp. $1569-1572$

\section{Detection of Pelvic Fractures Using Graph Cuts and Curvatures} Ananda Chowdhury (Jadavpur University, India); Joseph Burns (University of California, Irvine, USA); Bhaskar Sen (Jadavpur University, India); Arka Mukherjee (Jadavpur University, India); Jianhua Yao (National Institutes of Health, USA); Ronald Summers (National Institutes of Health, USA) pp. 1573-1576

\section{Automated Nuclei Clump Decomposition for Image Analysis in Neuronal Cell Fluorescent Microscopy}

Wei Xiong (Institute for Infocomm Research, Singapore); Shue Ching Chia (Institute of Infocomm Research, A*STAR, Singapore); Joo-Hwee Lim (Institute for Infocomm Research, Singapore) pp. $1577-1580$ 


\section{Automatic Labeling and Classification of Brain CT Images}

Tianxia Gong (School of Computing, National University of Singapore, Singapore); Shimiao Li (School of Computing, National University of Singapore, Singapore); Jie Wang (School of Computing, National University of Singapore, Singapore); Chew Lim Tan (National University of Singapore, Singapore); Boon Chuan Pang (National Neuroscience Institute, Tan Tock Seng Hospital, Singapore); Tchoyoson Lim (National Neuroscience Institute, Tan Tock Seng Hospital, Singapore); Cheng Kiang Lee (National Neuroscience Institute, Tan Tock Seng Hospital, Singapore); Qi Tian (Institute for Infocomm Research, Singapore); Zhuo Zhang (Institute for Infocomm Research, Singapore)

pp. 1581-1584

Histogram Analysis of CT Scans for Patients with Post-Mastectomy Lymphedema Maxine Tan (Vrije Universiteit Brussel, Belgium); Rudi Deklerck (VUB-ETRO, Belgium); An Tassenoy (Universitair Ziekenhuis Brussel (UZ Brussel), Belgium); Jan P.H. Cornelis (Vrije Universiteit Brussel, Belgium); Johan de Mey (Universitair Ziekenhuis Brussel (UZ Brussel), Belgium); Nico Buls (Universitair Ziekenhuis Brussel (UZ Brussel), Belgium)

pp. $1585-1588$

A GPU Accelerated Interactive Interface for Exploratory Functional Connectivity Analysis of fMRI Data Anders Eklund (Linköping University, Sweden); Ola Friman (Fraunhofer MeVis, Germany); Mats Andersson (Linköping University, Sweden); Hans Knutsson (Linköping University, Sweden) pp. 1589-1592

Application of Computational Anatomy Methods to MRI Data for the Diagnosis of Alzheimer's Disease

Jan Veerman (Philips Research, The Netherlands); Octavian Soldea (Philips

Research, The Netherlands); Pratik Sahindrakar (Philips Research, The Netherlands); Yijun Wan (Eindhoven University of Technology, The Netherlands); Radu Jasinschi (Philips Research, The Netherlands) pp. 1593-1596

\section{Medical Image Denoising Using Kernel Ridge Regression}

Dinh Hoan Trinh (Université Paris 13 \& LAGA, France); Marie Luong (Université Paris 13, France); Jean-Marie Rocchisani (Université Paris 13, France); Duong Pham (VAST, Vietnam); Françoise Dibos (LAGA, Universite Paris 13, France) pp. 1597-1600

\section{Extended Whole Mesh Deformation Model: Full 3D Processing}

Przemyslaw Lenkiewicz (Max Planck Institute for Psycholinguistics, The Netherlands); Manuela Pereira (University of Beira Interior, Portugal); Mario M. Freire (University of Beira Interior, Portugal); Jose Fernandes (Microsoft Portugal, Portugal) pp. 1601-1604

\section{Automatic Boundary Detection and Symmetry Calculation in Dermoscopy Images of Skin Lesions \\ Nikolay Metodiev Sirakov (Texas A\&M University-Commerce, USA); Mutlu Mete (Texas A\&M University-Commerce, USA); Nara Chakrader (Texas A\&M University- Commerce, USA) pp. $1605-1608$}




\title{
Cancer Detection From Biopsy Images Using Probabilistic and Discriminative
} Features

Atsushi Yaguchi (Tokyo University of Science, Japan); Takumi Kobayashi (National Institute of Advanced Industrial Science and Technology, Japan); Kenji Watanabe (National Institute of Advanced Industrial Science and Technology, Japan); Kenji Iwata (National Institute of Advanced Industrial Science and Technology, Japan); Tadaaki Hosaka (Tokyo University of Science, Japan); Nobuyuki Otsu (National Institute of Advanced Industrial Science and Technology, Japan) pp. 1609-1612

An Effective Method of Searching for Subregions in Chromosome Images Toru Abe (Cyberscience Center, Tohoku University, Japan); Chieko Hamada (Tohoku University, Japan)

pp. 1613-1616

\author{
A 3D Human Teeth Database Construction Based on a Point-Based Shape \\ Registration \\ Dongqing Chen (University of Louisville, USA) \\ pp. $1617-1620$
}

An Ensemble-based Microaneurysm Detector for Retinal Images

Bálint Antal (University of Debrecen, Hungary); Andras Hajdu (University of Debrecen, Hungary)

pp. 1621-1624

\section{TA.PE: Video Coding and Motion Estimation (Poster)}

\section{Optimal Rate Adaptation with Integer Linear Programming in the Scalable Extension} of H.264/AVC

Livio Lima (University of Brescia, Italy); Massimo Mauro (University of Brescia, Italy);

Tea Anselmo (STMicroelectronics, Italy); Daniele Alfonso (STMicroelectronics, Italy);

Riccardo Leonardi (University of Brescia, Italy)

pp. $1625-1628$

Rate-Distortion Analysis of Super-Resolution Image/Video Decoding

Keita Takahashi (The University of Tokyo, Japan); Takeshi Naemura (Tokyo University, Japan); Masayuki Tanaka (Tokyo Institute of Technology, Japan) pp. 1629-1632

\section{Temporal Trajectory Filtering for Bi-Directional Predicted Frames}

Marko Esche (Technische Universität Berlin, Germany); Andreas Krutz (Technische Universität Berlin, Germany); Alexander Glantz (Technische Universität Berlin, Germany); Thomas Sikora (Technische Universität Berlin, Germany) pp. $1633-1636$

\section{Chroma Intra Prediction Using Template Matching with Reconstructed Luma Components}

Chuohao Yeo (Institute for Infocomm Research, Singapore); Yih Han Tan (Institute for Infocomm Research, Singapore); Zhengguo Li (Institute for Inforcomm Research, Singapore); Susanto Rahardja (Institute for Infocomm Research, Singapore) pp. 1637-1640 
Practical Rate Control Algorithm for Temporal Scalability in Scalable Video Coding Jiaying Liu (Peking University, P.R. China); Yongjin Cho (University of Southern California, USA); Zongming Guo (Peking University, P.R. China)

pp. 1641-1644

Intra-WZ Quantization Mismatch in Distributed Video Coding

Jürgen Slowack (Ghent University, Belgium); Jozef Skorupa (Ghent University - IBBT, Belgium); Peter Lambert (Ghent University - IBBT, Belgium); Rik Van de Walle (Ghent University - IBBT, Belgium); Nikos Deligiannis (Vrije Universiteit Brussel - IBBT, Belgium); Adrian Munteanu (Vrije Universiteit Brussel, Belgium) pp. $1645-1648$

\section{Segmentation-Based Motion Compensation for Enhanced Video Coding}

Simone Milani (University of Padova, Italy); Giancarlo Calvagno (University of Padova, Italy)

pp. 1649-1652

Visual Perception Based Lagrangian Rate Distortion Optimization for Video Coding Xi Wang (Institute Of Computing Technology, Chinese Academy of Sciences, P.R. China); Li Su (Graduate School of Chinese Academy of Sciences, P.R. China); Qingming Huang (Graduate School of Chinese Academy of Sciences, P.R. China); Chunxi Liu (Graduate University of Chinese Academy of Sciences, CAS, P.R. China) pp. $1653-1656$

\section{SSIM-inspired Divisive Normalization for Perceptual Video Coding}

Shiqi Wang (Institute of Digital Media, School of Electronic Engineering and Computer Science, Peking University \& Department of Electrical and Computer Engineering, University of Waterloo, Canada); Abdul Rehman (University of Waterloo, Canada); Zhou Wang (University of Waterloo, Canada); Siwei Ma (University of Southern California, USA); Wen Gao (ICT-ISVISION Joint R\&D Laboratory for Face Recognition, CAS, P.R. China) pp. $1657-1660$

Combining Open- and Closed-Loop Architectures for H.264/AVC-to-SVC Transcoding Sebastiaan Van Leuven (Ghent University - IBBT, Belgium); Jan De Cock (Ghent University - IBBT, Belgium); Glenn Van Wallendael (Ghent University - IBBT, Belgium); Rik Van de Walle (Ghent University - IBBT, Belgium); Rosario GarridoCantos (University of Castilla-La Mancha, Spain); Jose Luis Martínez (Universidad Complutense de Madrid, Spain); Pedro Cuenca (University of Castill La Mancha, Spain) pp. 1661-1664

Adaptive Intra Modes Reduction by Clustering for H.264/AVC Weijia Zhu (Beijing University of Technology, P.R. China); Wenpeng Ding (Beijing University of Technology, P.R. China); Yunhui Shi (Beijing University of Technology, P.R. China); Yanfeng Sun (Beijing University of Technology, P.R. China); Baocai Yin (Beijing University of Technology, P.R. China) pp. 1665-1668

Efficient P-frame Complexity Estimation for Frame Layer Rate Control of H.264/AVC Kairan Sun (Fudan University, P.R. China); Bo Yan (Fudan University, P.R. China) pp. $1669-1672$ 
Decoder Side True Motion Estimation for Very Low Bitrate B-frame Coding Hasan F Ates (Isik University, Istanbul, Turkey); Burak Cizmeci (Technische Universitaet Muenchen, Germany) pp. $1673-1676$

Motion Compensated Prediction Using Partial Mesh Generation Han Huang (Beijing Jiaotong University, P.R. China); John W. Woods (Rensselaer Polytechnic Institute, USA); Yao Zhao (Beijing Jiaotong University, P.R. China) pp. $1677-1680$

High Definition Video Intra-Only Coding Based on Node-Cell Macroblock Pixel Structure and 2-D Interleaved DCT

Dong Zheng (Communications Research Center, Canada); Demin Wang (Communications Research Center, Canada); Liang Zhang (Communications Research Centre Canada, Canada) pp. 1681-1684

Mode Dependent Dct/Dst for Intra Prediction in Block-Based Image/Video Coding Ankur Saxena (Samsung Telecommunications America, USA); Felix Fernandes (Samsung Electronics Co., Ltd, USA) pp. $1685-1688$

TA.PF: Color/Multispectral Imaging and Rendering (Poster)

Modeling and Application of Color Noise Perception Dependent on Background Color and Spatial Frequency

Makoto Shohara (Japan Advanced Institute of Science and Technology, Japan);

Kazunori Kotani (JAIST, Japan)

pp. 1689-1692

\section{Compressive Demosaicing for Periodic Color Filter Arrays}

Mohammad Aghagolzadeh (Michigan State University, USA); Abdolreza

Abdolhosseini Moghadam (Michigan State University, USA); Mrityunjay Kumar (Eastman Kodak Company, USA); Hayder Radha (Michigan State University, USA) pp. 1693-1696

\section{Color Distribution Matching Using a Weighted Subspace Descriptor}

Kenjiro Sugimoto (Waseda University, Japan); Sei-ichiro Kamata (Waseda University, Japan)

pp. $1697-1700$

Real-time Visual Saliency by Division of Gaussians

loannis Katramados (TRW Conekt, United Kingdom); Toby Breckon (Cranfield University, United Kingdom)

pp. 1701-1704

NEURAL GRAY EDGE: Improving Gray Edge Algorithm Using Neural Network Mohsen Ebrahimi Moghaddam (Shahid Beheshti University, Iran) pp. $1705-1708$ 
Dimensionality Reduction of Hyperspectral Images with Wavelet Based Empirical Mode Decomposition

Esra Tunc Gormus (University of Bristol, United Kingdom); Nishan Canagarajah (University of Bristol, United Kingdom); Alin M Achim (University of Bristol, United Kingdom)

pp. $1709-1712$

Removing Shadows From Images Using Color and Near-infrared Neda Salamati (EPFL, Switzerland); Arthur Germain (EPFL, Switzerland); Sabine Süsstrunk (EPFL, Switzerland) pp. 1713-1716

Inverse Halftoning with Nonlocal Regularization Xin Li (West Virginia University, USA) pp. $1717-1720$

Electro-Photographic Model Based Stochastic Clustered-Dot Halftoning with Direct Binary Search Puneet Goyal (Purdue University, USA); Madhur Gupta (Purdue University, USA); Carl Staelin (Hewlett-Packard Laboratories, Israel); Mani Fischer (Hewlett-Packard Laboratories, Israel); Omri Shacham (Hewlett-Packard Indigo, Israel); Tamar Kashti (Hewlett-Packard Indigo, Israel); Jan Allebach (Purdue University, USA) pp. 1721-1724

High Resolution Subpixel and Subframe Rendering for Color Flatpanel and Projector Displays

Keigo Hirakawa (University of Dayton, USA); Jing Gu (Kingway Technology Shanghai Ltd., P.R. China)

pp. $1725-1728$

\section{Color Quantization Using C-Means Clustering Algorithms}

M. Emre Celebi (Louisiana State University in Shreveport, USA); Quan Wen (University of Electronic Science and Technology of China, P.R. China); Juan Chen (University of Electronic Science and Technology of China, P.R. China) pp. 1729-1732

Design of Color Screen Sets for Robustness to Color Plane Misregistration Jin-Young Kim (Purdue University, USA); Yung-Yao Chen (Purdue University, USA); Mani Fischer (Hewlett-Packard Laboratories, Israel); Omri Shacham (Hewlett-Packard Indigo, Israel); Carl Staelin (Hewlett-Packard Laboratories, Israel); Jan Allebach (Purdue University, USA) pp. $1733-1736$

\section{TA.PG: Applications of Image \& Video Interpretation and Understanding (Poster)}

Face Recognition Using Maximum Local Fisher Discriminant Analysis

Lei Wang (Xidian University, P.R. China); Hongbing Ji (School of Electronic

Engineering, Xidian University, P.R. China); Ya Shi (Xidian University, P.R. China) pp. $1737-1740$ 


\section{A Non-Temporal Texture Driven Approach to Real-time Fire Detection}

Audrey Chenebert (Cranfield University, United Kingdom); Toby Breckon (Cranfield University, United Kingdom); Anna Gaszczak (Cranfield University, United Kingdom) pp. $1741-1744$

\section{Temporal Trimap Propagation for Video Matting Using Inferential Statistics} Muhammad Sarim (University of Surrey, United Kingdom) pp. $1745-1748$

\section{A Biologically Inspired System for Fast Handwritten Digit Recognition} Zhe Wang (Beijing Jiaotong University, P.R. China); Yaping Huang (Beijing Jiaotong University, P.R. China); Siwei Luo (Beijing Jiaotong University, P.R. China); Liang Wang (Beijing Jiaotong University, P.R. China) pp. $1749-1752$

\section{Joint Optimization of Background Subtraction and Object Detection for Night \\ Surveillance}

Congcong Li (Cornell University, USA); Chih-Wei Lin (Industrial Technology Research Institute, Taiwan); Shiaw-Shian Yu (Industrial Technology Research Institute, Taiwan); Tsuhan Chen (Cornell University, USA) pp. 1753-1756

\section{Automatic Bandwidth Estimation Strategy for High-Quality Non-Parametric Modeling} Based Moving Object Detection

Carlos Cuevas (Universidad Politécnica de Madrid, Spain); Narciso García

(Universidad Politécnica de Madrid, Spain) pp. $1757-1760$

Face Recognition Through Regional Weight Estimation Daniel Yule (University of Northern British Columbia, Canada); Liang Chen (University of N. British Columbia, Canada) pp. $1761-1764$

Ultrasound Video Analysis for Understanding Infant Breastfeeding Gianluca Monaci (Philips research, The Netherlands); Mike Woolridge (University of Leeds, United Kingdom) pp. $1765-1768$

\section{Exploiting Feature Correspondence Constraints for Image Recognition} Linbo Wang (Nanjing University, P.R. China); Feng Tang (Hewlett-Packard Laboratories, USA); Yanwen Guo (Nanjing University, P.R. China); Suk Hwan Lim (HP Labs, USA); Nelson L. Chang (Hewlett-Packard Laboratories, USA) pp. 1769-1772

\section{Sparse Cost-sensitive Classifier with Application to Face Recognition} Jiangyue Man (Nanjing University of Posts and Telecommunications, P.R. China); Xiaoyuan Jing (College of Automation, Nanjing University of Posts and Telecommunications \& State Key Laboratory for Software Engineering, Wuhan University, P.R. China); David Zhang (The Hong Kong Polytechnic University, Hong Kong); Chao Lan (Nanjing University of Posts and Telecommunications, P.R. China) pp. 1773-1776

Hierarchical Bag-of-Features for Hand Posture Recognition Yuelong Chuang (Zhejiang University, P.R. China) pp. $1777-1780$ 
Context-driven Moving Object Detection in Aerial Scenes with User Input

Christophe Guilmart (ENS Cachan \& Onera, France); Stéphane Herbin (Onera, France); Patrick Perez (Technicolor, France) pp. 1781-1784

Human Face Classification Based on Localized Blur Descriptors Abdul Adeel Mohammed (University of Waterloo, Canada); Jonathan Wu (University of Windsor, Canada); Maher Sid-Ahmed (University of Windsor, Canada) pp. $1785-1788$

Combining Global and Local Features for Food Identification in Dietary Assessment Marc Bosch (Purdue University, USA); Fengqing Zhu (Purdue University, USA); Nitin Khanna (Purdue University, USA); Carol Boushey (Purdue University, USA); Ed Delp (Purdue University, USA) pp. 1789-1792

Human Detection Using Multi-camera and 3D Scene Knowledge Chengbin Zeng (Beijing University of Posts and Telecommunications, P.R. China); Huadong Ma (Beijing University of Posts and Telecommunications, P.R. China) pp. $1793-1796$

Multimodal Learning for Multi-Label Image Classification Yanwei Pang (Tianjin University, P.R. China); Zhao Ma (Tianjin University, P.R. China); Yuan Yuan (Chinese Academy of Sciences, P.R. China); Xuelong Li (Xi'an Institute of Optics and Precision Mechanics, Chinese Academy of Sciences, P.R. China); Kongqiao Wang (Nokia Research Center, P.R. China) pp. $1797-1800$

TP.L1: Distributed Compression: Multimedia Applications (Special Session)

Distributed Compression: Overview of Current and Emerging Multimedia Applications Lina Stankovic (University of Strathclyde, United Kingdom); Vladimir Stankovic (University of Strathclyde, United Kingdom); Samuel Cheng (University of Oklahoma, USA)

pp. 1801-1804

\section{Quality-Controlled View Interpolation for Multiview Video}

Mina Makar (Stanford University, USA); Yao-Chung Lin (Stanford University, USA); Ngai-Man Cheung (Stanford University, USA); Derek Pang (Stanford University, USA); Bernd Girod (Stanford University, USA) pp. $1805-1808$

A Comparison of the Error Resiliency of Bit-Plane Based and Symbol Based PixelDomain Distributed Video Coding Hu Chen (Technische Universität München, Germany); Eckehard Steinbach (Munich University of Technology, Germany); Chang Wen Chen (State University of New York at Buffalo, USA)

pp. $1809-1812$

\section{Distributed Coding of Endoscopic Video}

Nikos Deligiannis (Vrije Universiteit Brussel - IBBT, Belgium); Frederik Verbist (Vrije Universiteit Brussel - IBBT, Belgium); Joeri Barbarien (Vrije Universiteit Brussel, 
Belgium); Jürgen Slowack (Ghent University, Belgium); Rik Van de Walle (Ghent University - IBBT, Belgium); Peter Schelkens (Vrije Universiteit Brussel, Belgium); Adrian Munteanu (Vrije Universiteit Brussel, Belgium) pp. 1813-1816

break

Wyner-Ziv Coding for Depth Maps in Multiview Video-Plus-Depth

Giovanni Petrazzuoli (Télécom ParisTech, France); Marco Cagnazzo (TELECOM ParisTech, France); Frederic Dufaux (Telecom Paristech \& CNRS, France); Beatrice Pesquet-Popescu (Télécom ParisTech, France) pp. $1817-1820$

\section{Distributed Compression of Zerotrees of Wavelet Coefficients} Yige Wang (MERL, USA); Shantanu Rane (Mitsubishi Electric Research Laboratories, USA); Petros T Boufounos (MERL \& Rice University, USA); Anthony Vetro (Mitsubishi Electic Research Laboratories, USA) pp. 1821-1824

Distributed Source Coding for Securing a Hand-Based Biometric Recognition System Mauricio Ramalho (Instituto de Telecomunicações, Portugal); Paulo Lobato Correia (Instituto Superior Tecnico - Universidade Tecnica Lisboa \& Instituto de Telecomunicacoes, Portugal); Luis Ducla Soares (I.S.C.T.E. / I.T. - Lisbon, Portugal) pp. $1825-1828$

\section{Distributed Transforms for Efficient Data Gathering in Arbitrary Networks} Javier Trufero (University of Southern California, USA); Sunil K. Narang (University of Southern California, USA); Antonio Ortega (USC, USA) pp. 1829-1832

\section{TP.L2: Image and Noise Models for Restoration (Lecture)}

\section{Resolution-invariant Separable ARMA Modeling of Images}

Aurélien Bourquard (Ecole Polytechnique Federale de Lausanne, Switzerland); Hagai Kirshner (EPFL, Switzerland); Michael Unser (EPFL, Switzerland) pp. 1833-1836

CONDY: Ultra-Fast High Performance Restoration Using Multi-Frame L2-Relaxed-LO Sparsity and Constrained Dynamic Heuristics Javier Portilla (Consejo Superior de Investigaciones Científicas, Spain); Elena GilRodrigo (Consejo Superior de Investigaciones Científicas, Spain); David Miraut (Universidad Rey Juan Carlos, Spain); Ricardo Suarez-Mesa (Universidad Rey Juan Carlos, Spain) pp. $1837-1840$

\section{Sparsity-based Image Deblurring with Locally Adaptive and Nonlocally Robust} Regularization

Xin Li (West Virginia University, USA); Weisheng Dong (Xidian University, P.R. China); Guangming Shi (Xidian University, P.R. China); Lei Zhang (The Hong Kong Polytechnic University, Hong Kong) pp. 1841-1844 


\section{Patch Similarity Under Non Gaussian Noise}

Charles-Alban Deledalle (Telecom ParisTech \& Institut Telecom, CNRS LTCI, France); Florence Tupin (Télécom Paris, France); Loïc Denis (Centre de Recherche Astrophysique de Lyon, France) pp. $1845-1848$

break

\section{Generalized Subspace Based High Dimensional Density Estimation}

Karthikeyan Shanmuga Vadivel (University of California Santa Barbara, USA); Mehmet Emre Sargin (Google Inc., USA); Swapna Joshi (UCSB, USA); Bangalore Manjunath (UCSB, USA); Scott Grafton (UCSB, USA) pp. $1849-1852$

Ising Field Parameter Estimation From Incomplete and Noisy Data Jean-François Giovannelli (IMS, UMR CNRS 52 18, Université Bordeaux 1, France) pp. $1853-1856$

\section{Noise Estimation Using Statistics of Natural Images}

Guangtao Zhai (McMasster University, Canada); Xiaolin Wu (McMaster University, Canada)

pp. $1857-1860$

\section{Hazy Image Modeling Using Color Ellipsoids}

Kristofor Gibson (University of California San Diego, USA); Truong Nguyen (University of California in San Diego, USA) pp. 1861-1864

\section{TP.L3: Motion Analysis and Object Tracking (Lecture)}

\section{A Fast Object Tracking Approach Based on Sparse Representation}

Zhenjun Han (Graduate University of Chinese Academy of Sciences, P.R. China); Jianbin Jiao (Graduate University of Chinese Academy of Sciences, P.R. China); Qixiang Ye (Graduate University of Chinese Academy of Sciences, P.R. China) pp. $1865-1868$

\section{Efficiently Selecting Spatially Distributed Keypoints for Visual Tracking} Steffen Gauglitz (University of California, Santa Barbara, USA); Luca Foschini (University of California, Santa Barbara, USA); Matthew Turk (University of California, Santa Barbara, USA); Tobias Höllerer (University of California, Santa Barbara, USA) pp. $1869-1872$

Simultaneous 3D Object Tracking and Camera Parameter Estimation by Bayesian Methods and Transdimensional MCMC Sampling

Raúl Mohedano (Universidad Politécnica de Madrid, Spain); Narciso García (Universidad Politécnica de Madrid, Spain) pp. 1873-1876

Multi-person Tracking Based on Vertical Reference Lines and Dynamic Visibility Analysis

Xinghan Luo (Utrecht University, The Netherlands); Robby Tan (Utrecht University, The Netherlands); Remco C. Veltkamp (Utrecht University, The Netherlands) pp. $1877-1880$ 
break

Local Complexity Adaptable Trajectory Partitioning Via Minimum Message Length Charles Twardy (George Mason University, USA); Anthony Stefanidis (George Mason University, USA)

pp. 1881-1884

\section{Fast TV-L1 Optical Flow for Interactivity}

Emmanuel d'Angelo (EPFL \& Signal Processing Lab 2, Switzerland); Johan Paratte (EPFL, Switzerland); Gilles Puy (EPFL, Switzerland); Pierre Vandergheynst (EPFL, Switzerland)

pp. $1885-1888$

\section{Co-occurrence Flow for Pedestrian Detection}

Atsuto Maki (Toshiba Research Europe, United Kingdom); Akihito Seki (Toshiba Corporation, Japan); Tomoki Watanabe (Toshiba Corporation, Japan); Roberto Cipolla (University of Cambridge, United Kingdom)

pp. 1889-1892

\section{Hierarchical Fusion of Descriptor Matching and L-K Optical Flow}

Haibo Wang (Institute of Automation, Chinese Academy of Sciences, P.R. China); Chunhong Pan (Institute of Automation, Chinese Academy of Sciences, P.R. China); Franck Davoine (CNRS, P.R. China); ShaoGuo Liu (Institute of Automation, Chinese Academy of Sciences, P.R. China) pp. $1893-1896$

\section{TP.L4: Sparse Image Recovery (Lecture)}

\section{Increasing Imaging Resolution by Covering Your Sensor}

Michael Schöberl (University of Erlangen-Nuremberg, Germany); Jürgen Seiler (University of Erlangen-Nuremberg, Germany); Siegfried Foessel (Fraunhofer IIS, Germany); Andre Kaup (University of Erlangen-Nuremberg, Germany) pp. 1897-1900

Inverse Problems with Poisson Noise: Primal and Primal-Dual Splitting Francois-Xavier Dupé (CEA, France); Jalal Fadili (GREYC CNRS UMR 6072, ensicaen, France); Jean-Luc Starck (CEA, France) pp. 1901-1904

\section{Sparse Image Restoration Using Iterated Linear Expansion of Thresholds} Hanjie Pan (The Chinese University of Hong Kong, Hong Kong); Thierry Blu (EPFL, Switzerland) pp. 1905-1908

\section{Cutset Sampling and Reconstruction of Images} Ashish Farmer (University of Michigan, USA); Awlok Josan (University of Michigan, USA); Matthew Prelee (University of Michigan, USA); David L Neuhoff (University of Michigan, USA); Thrasyvoulos N. Pappas (Northwestern University, USA) pp. 1909-1912

break 


\section{A Graph Cut Method for Linear Inverse Problems}

Ahmet Tuysuzoglu (Boston University, USA); Ivana Stojanovic (Boston University, USA); David Castanon (Boston University, USA); William Karl (Boston University, USA)

pp. 1913-1916

\section{Convex Approaches to Model Wavelet Sparsity Patterns}

Nikhil Rao (University Of Wisconsin Madison, USA); Rob Nowak (University of Wisconsin, Madison, USA); Stephen J Wright (University of Wisconsin, USA); Nick Geoffrey Kingsbury (University of Cambridge, United Kingdom) pp. 1917-1920

\section{Weighted Fidelity in Non-Uniformly Quantized Compressed Sensing}

Laurent Jacques (University of Louvain, Belgium); David Hammond (University of Oregon, USA); Jalal Fadili (GREYC CNRS UMR 6072, ensicaen, France) pp. 1921-1924

\section{Refractive Index Estimation Using Photometric Stereo}

Gule Saman (University of York, United Kingdom); Edwin Hancock (University of York, United Kingdom)

pp. 1925-1928

\section{TP.L5: Image Forensics (Lecture)}

\section{Detection of Non-Aligned Double JPEG Compression with Estimation of Primary \\ Compression Parameters}

Tiziano Bianchi (University of Firenze, Italy); Alessandro Piva (University of Florence,

Italy)

pp. 1929-1932

\section{Identifying Computer Generated Graphics Via Histogram Features}

Ruoyu Wu (Institute of Computer Science and Technology, Peking University, P.R.

China); Xiaolong Li (Institute of Computer Science and Technology, Peking University,

P.R. China); Bin Yang (Institute of Computer Science and Technology, Peking University, P.R. China)

pp. 1933-1936

Eye Specular Highlights Telltales for Digital Forensics: a Machine Learning Approach Priscila Saboia (State University of Campinas, Brazil); Tiago Carvalho (University of Campinas (Unicamp), Brazil); Anderson Rocha (State University of Campinas, Brazil) pp. $1937-1940$

\section{An Application of Sparse Code Shrinkage to Image Steganalysis Based on Supervised Learning \\ Michiharu Niimi (Kyushu Institute of Technology, Japan); Hideki Noda (Kyushu Institute of Technology, Japan) \\ pp. 1941-1944}

break 
Modeling the EXIF-Image Correlation for Image Manipulation Detection

Jiayuan Fan (Nanyang Technological University, Singapore); Alex Kot (Nanyang Technological University, Singapore); Hong Cao (Nanyang Technological University, Singapore); Farook Sattar (University of Victoria, Canada) pp. 1945-1948

\section{Countering JPEG Anti-Forensics}

Giuseppe Valenzise (Politecnico di Milano, Italy); Vitaliano Nobile (Politecnico di Milano, Italy); Marco Tagliasacchi (Politecnico di Milano, Italy); Stefano Tubaro (Politecnico di Milano, Italy) pp. 1949-1952

\section{Exploring Compression Effects for Improved Source Camera Identification Using} Strongly Compressed Video

Wei-Hong Chuang (University of Maryland, USA); Hui Su (University of Maryland, USA); M Wu (University of Maryland, USA) pp. 1953-1956

\section{Passive Spread-Spectrum Steganalysis}

Ming Li (State University of New York at Buffalo, USA); Michel Kulhandjian (State University of New York at Buffalo, USA); Dimitris A. Pados (State University of New York at Buffalo, USA); Stella N. Batalama (State University of New York at Buffalo, USA); Michael Medley (Air Force Research Laboratory, USA) pp. 1957-1960

\section{TP.L6: 3D Image Generation and Analysis (Lecture)}

\section{D to 3D Conversion of Sports Content Using Panoramas}

Lars Schnyder (Disney Research Zurich, Switzerland); Oliver Wang (Disney Research Zurich, Switzerland); Aljoscha Smolic (Disney Research Zurich, Switzerland) pp. 1961-1964

Hole Filling with Random Walks Using Occlusion Constraints in View Synthesis Sunghwan Choi (Yonsei University \& Digital Image Media Laboratory, Korea); Bumsub Ham (Yonsei University, Korea); Kwang Hoon Sohn (Yonsei University, Korea) pp. 1965-1968

\section{A Triangular-Warping Based View Synthesis Scheme with Enhanced Artifact Reduction for FTV \\ Chao-Hsuan Li (National Chiao Tung University, Taiwan); Hsueh-Ming Hang (NCTU, Taiwan) \\ pp. 1969-1972}

\section{Super-Resolution Plane Sweeping for Free-Viewpoint Image Synthesis}

Keita Takahashi (The University of Tokyo, Japan); Masato Ishii (NEC Corporation, Japan); Takeshi Naemura (Tokyo University, Japan) pp. 1973-1976

break 
Depth Map Reconstruction Using Color-based Region Merging

Camilo Dorea (University of Brasilia, Brazil); Ricardo L de Queiroz (University of Brasil, Brazil)

pp. 1977-1980

Recovering Depth From a Single Image Using Spectral Energy of the Defocused Step Edge Gradient

Cheng-Wei Chen (National Taiwan University, Taiwan); Yung-Yaw Chen (National Taiwan University, Taiwan)

pp. 1981-1984

\section{D Surface Registration Using Z-SIFT}

Lulu He (Northwestern University, USA); Sen Wang (Eastman Kodak Company, USA); Thrasyvoulos N. Pappas (Northwestern University, USA) pp. 1985-1988

\section{TP.L7: Lossless and Predictive Coding (Lecture)}

\section{L_2 Restoration of I_linfty-decoded Images with Context Modeling}

Jiantao Zhou (McMaster University, Canada); Xiaolin Wu (McMaster University, Canada)

pp. 1989-1992

Image Similarity Using the Normalized Compression Distance Based on Finite Context Models

Armando J Pinho (University of Aveiro, Portugal); Paulo Ferreira (University of Aveiro, Portugal)

pp. 1993-1996

On Lossless Image Compression Using the Burrows-Wheeler Transform Donald Adjeroh (West Virginia University, USA); Kalyan Bhupathiraju (West Virginia University, USA)

pp. 1997-2000

Exploitation of Context Classification for Parallel Pixel Coding in JPEG-LS Simeon Wahl (University of Stuttgart, Germany); Haitham Tantawy (University of Stuttgart, Germany); Zhe Wang (University of Stuttgart, Germany); Philipp Werner (University of Stuttgart, Germany); Sven Simon (University of Stuttgart, Germany) pp. 2001-2004

break

Improved H.264/AVC Lossless Intra Compression Using Multiple Partition Prediction for 4X4 Intra Block

Sang Heon Lee (Seoul Nat'I University, Korea); Jewoong Ryu (Seoul National University, Korea); Nam-Ik Cho (Seoul National University, Korea)

pp. 2005-2008

Improved Lossless Coding Algorithm in H.264/AVC Based on Hierarchical Intra Prediction

Li-Li Wang (Hong Kong Polytechnic University, Hong Kong); Wan-Chi Siu (The Hong Kong Polytechnic University, Hong Kong) pp. 2009-2012 
Adaptive Least Squares Prediction for Stereo Image Coding

Luís Lucas (Polytechnic Institute of Leiria \& Institute of Telecommunications,

Portugal); Nuno Rodrigues (IPL/Institute of Telecommunications, Portugal); Eduardo

Silva (UFRJ, Brazil); Sérgio M. M. Faria (Institute of Telecommunications \&

Polytechnic Institute of Leiria, Portugal)

pp. 2013-2016

\title{
TP.L8: Ultrasound and Microscopic Imaging (Lecture)
}

\author{
Elastographic Image Reconstruction: A Stochastic State Space Approach \\ Jun Wang (Zhejiang University, P.R. China) \\ pp. 2017-2020
}

\section{Reducing the Training Set Using Semi-Supervised Self-Training Algorithm for Segmenting the Left Ventricle in Ultrasound Images} Jacinto C. Nascimento (Instituto de Sistemas e Robotica, Portugal); Gustavo Carneiro (Instituto de Sistemas e Robótica (ISR), Portugal) pp. 2021-2024

\section{Enhanced Classification of Focal Hepatic Lesions in Ultrasound Images Using Novel Texture Features}

Sihyoung Lee (Korea Advanced Institute of Science and Technology, Korea); In A Jo (Korea Advanced Institute of Science and Technology, Korea); Kyung Won Kim (Seoul National University Hospital, Korea); Jae Young Lee (Seoul National University Hospital, Korea); Yong Man Ro (KAIST, Korea) pp. 2025-2028

\section{Realistic Log-Compressed Law for Ultrasound Image Recovery} Gonzalo Vegas-Sánchez-Ferrero (University of Valladolid, Spain); Diego MartínMartínez (University of Valladolid, Spain); Pablo Casaseca-de-la-Higuera (University of Valladolid, Spain); Lucilio Cordero-Grande (University of Valladolid, Spain); Santiago Aja-Fernández (Universidad de Valladolid, Spain); Marcos MartínFernández (Universidad de Valladolid, Spain); Cesar Palencia (University of Valladolid, Spain) pp. 2029-2032

break

\section{Compact Rotation Invariant Image Descriptors by Spectral Trimming} Maxime Taquet (Belgium \& Computational Radiology Laboratory, Harvard Medical School, Belgium); Laurent Jacques (University of Louvain, Belgium); Benoit Macq (UCL, Belgium); Sylvain Jaume (Massachusetts Institute of Technology \& Harvard Medical School, USA) pp. 2033-2036

\section{Modeling of PSF for Refractive Index Variation in Fluorescence Microscopy} Sameer Hiware (Indian Institute of Technology Bombay, India); Pradyot Porwal (Indian Institute of Management Bangalore \& Indian Institute of Technology Bombay, India); Rajbabu Velmurugan (IIT Bombay, India); Subhasis Chaudhuri (Indian Institute of Technology, Bombay, India) pp. 2037-2040 
Image Filtering Using Anisotropic Structure Tensor for Cell Membrane Enhancement in 3D Microscopy

Sorin Pop (Institut Pasteur, France); Alexandre Dufour (Institut Pasteur, France); Jean-Christophe Olivo-Marin (Institut Pasteur, France) pp. 2041-2044

ALL-IDB: The Acute Lymphoblastic Leukemia Image Database for Image Processing Ruggero Donida Labati (Università degli Studi di Milano, Italy); Vincenzo Piuri (University of Milan, Italy); Fabio Scotti (Universita' degli Studi di Milano, Italy) pp. 2045-2048

\section{TP.L9: Object and Human Detection (Lecture)}

Detecting Humans Under Occlusion Using Variational Mean Field Method

Thanh Duc Nguyen (University of Wollongong, Australia); Philip Ogunbona (University of Wollongong, Australia); Wanqing Li (University of Wollongong, Australia) pp. 2049-2052

Local Binary Pattern Features for Pedestrian Detection At Night/Dark Environment Yunyun Cao (Security \& Safety Systems Development Office \& Tokyo Research \& Development Center, Panasonic Corporation, Japan); Sugiri Pranata (Panasonic Singapore Laboratories Pte Ltd, Singapore); Hirofumi Nishimura (Tokyo R\&D Center, Panasonic Corporation, Japan) pp. 2053-2056

\section{Effective Discretization of Gabor Features for Real-time Face Detection} Feijun Jiang (Hong Kong University of Science and Technology, P.R. China); Bertram Shi (Hong Kong University of Science and Technology, Hong Kong); Mika Fischer (Karlsruhe Institute of Technology (KIT), Germany); Hazim Ekenel (Karlsruhe Institute of Technology (KIT), Germany) pp. 2057-2060

\section{Robust Crowd Counting Using Detection Flow}

Junliang Xing (Tsinghua University, P.R. China); Haizhou Ai (Tsinghua University, P.R. China); Liwei Liu (Tsinghua University, P.R. China); Shihong Lao (OMRON Corporation, Japan) pp. 2061-2064

break

Direction-based Stochastic Matching for Pedestrian Recognition in Non- Overlapping Cameras

Xiaotang Chen (Institute of Automation, Chinese Academy of Science, P.R. China); Kaiqi Huang (Chinese Academy of Sciences, P.R. China); Tieniu Tan (NLPR, P.R. China) pp. 2065-2068

Robust Shape-From-Image-Focus by 3-D Multivariate Statistical Analyses Mathieu Fernandes (Ecole des Mines de Saint-Etienne, France); Yann Gavet (Ecole des Mines de Saint-Etienne, France); Jean-Charles Pinoli (Ecole Nationale Supérieure des Mines, France) pp. 2069-2072 


\section{TP.PA: Image \& Video Representations and Applications (Poster)}

\section{Robust View Transformation Model for Gait Recognition}

Shuai Zheng (Institute of Automation, Chinese Academy of Sciences, P.R. China); Junge Zhang (Institute of Automation, Chinese Academy of Sciences, P.R. China); Kaiqi Huang (Chinese Academy of Sciences, P.R. China); Ran He (Institute of Antomation, Chines Academy of Sciences, P.R. China); Tieniu Tan (NLPR, P.R. China)

pp. 2073-2076

Incremental Orthogonal Projective Non-negative Matrix Factorization and Its Applications

Dong Wang (Dalian University of Technology, P.R. China); Huchuan Lu (Dalian University of Technology, P.R. China)

pp. 2077-2080

\section{Error Concealment Via 3-Mode Tensor Approximation}

Dzung Nguyen (Johns Hopkins University \& Johns Hopkins Univ, USA); Minh Dao (Johns Hopkins University, USA); Trac D. Tran (Johns Hopkins University, USA) pp. 2081-2084

\section{Relative Depth From Monocular Optical Flow}

Enric Meinhardt-Llopis (Fundació Barcelona Media \& Universitat Pompeu Fabra, Spain); Olivier D'Hondt (Barcelona Media, Spain); Gabriele Facciolo (Universitat Pompeu Fabra, Spain); Vicent Caselles (Universitat Pompeu Fabra, Spain) pp. 2085-2088

\section{Vector Field Analysis for Motion Pattern Identification in Video}

Nandita Nayak (University of California, Riverside, USA); Ahmed T Kamal (University of California, Riverside, USA); Amit Roy-Chowdhury (University of California, Riverside, USA)

pp. 2089-2092

\section{Video Resolution Enhancement by Using Complex Wavelet Transform} Hasan Demirel (Eastern Mediterranean University, Turkey); Gholamreza Anbarjafari (Cyprus International University, Turkey); Cagri Ozcinar (University of Surrey, United Kingdom); Sara Izadpanahi (Eastern Mediterranean University, Turkey) pp. 2093-2096

Space-Time Template Matching for Human Action Detection Using Volume-Based Generalized Hough Transform YungChi Lo (National Taiwan Ocean University \& Lab 603, Taiwan); Po-Yen Lee (National Taiwan Ocean University, Taiwan); Shyi-Chyi Cheng (National Taiwan Ocean University, Taiwan) pp. 2097-2100

\section{An Improved Depth Map Estimation for Coding and View Synthesis} Quwen Zhang (Shanghai University \& School of Communication and Information Engineering, P.R. China)

pp. 2101-2104 
Multi-Scale 3D Representation Via Volumetric Quasi-Random Scale Space Akshaya Mishra (University of Waterloo, Canada); Alexander Wong (University of Waterloo, Canada); Paul Fieguth (University of Waterloo, Canada); David Clausi (University of Waterloo, Canada) pp. 2105-2108

Motion Re-estimation for H.264/AVC Video Downscaling Transcoding Using EPZS Algorithm

Chia-Tien Lin (National Central University, Taiwan); Yinyi Lin (National Central University, Taiwan, Taiwan) pp. 2109-2112

\section{Generalized Selective Data Pruning for Video Sequence}

Yuichi Tanaka (Utsunomiya University, Japan); Madoka Hasegawa (Utsunomiya University, Japan); Shigeo Kato (Utsunomiya University, Japan) pp. 2113-2116

HyperComplex Polar Fourier Analysis for Color Image Zhuo Yang (Waseda University, Japan); Sei-ichiro Kamata (Waseda University, Japan)

pp. 2117-2120

Analysis of Solder Paste Scooping with Hierarchical Point Processes Csaba Benedek (MTA SZTAKI \& Dept. of Electronic Technology, Budapest University of Technology and Economics, Hungary) pp. 2121-2124

\section{Image Segmentation with Hierarchical Topic Assignment} Hao Feng (Beijing University of Aeronautics and Astronautics, P.R. China); Zhiguo Jiang (Image Processing Center, Beijing University of Aeronautics and Astronautics, P.R. China) pp. 2125-2128

\section{Color Correction Via Robust Reference Selection and Recovery Using a Low-Rank} Matrix Model

Dong Li (The Hong Kong Polytechnic University, Hong Kong); Xudong Xie (Tsinghua University, P.R. China); Kenneth Lam (Hong Kong Polytechnic University, Hong Kong) pp. 2129-2132

\section{TP.PB: Biomedical Image Registration and Segmentation (Poster)}

\section{Weighting Function in Random Walk Based Left Ventricle Segmentation \\ Sarada Prasad Dakua (Indian Institute of Technology Guwahati, India) \\ pp. 2133-2136}

\section{Topological Vascular Tree Segmentation for Retinal Images Using Shortest Path Connection}

Li Chen (Wuhan University of Science and Technology, P.R. China); YaoYong Ju (Wuhan University of Science and Technology, P.R. China); Sheng Ding (Wuhan University of Science and Technology, P.R. China); XiaoMing Liu (Wuhan University of Science and Technology, P.R. China) pp. $2137-2140$ 


\section{D-GE Image Segmentation Based on Level-Sets}

Eleftheria Mylona (University of Athens, Greece); Michalis Savelonas (University of Athens, Greece); Dimitris Maroulis (University of Athens, Greece); Michalis Aivaliotis (Institute of Molecular Biology \& Foundation of Research and Technology, Greece) pp. 2141-2144

Multiresolution Localization and Segmentation of the Optical Disc in Fundus Images Using Inpainted Background and Vessel Information

Andrea Giachetti (University of Verona, Italy); Khai Chin (University of Dundee, United Kingdom); Emanuele Trucco (University of Dundee, United Kingdom); Caroline Cobb (NHS Ninewells Hospital, Dundee, United Kingdom); Peter Wilson (NHS Ninewells Hospital, Dundee, United Kingdom) pp. $2145-2148$

\section{Efficient Multi-Object Segmentation of 3D Medical Images Using Clustering and Graph} Cuts

Razmig Kéchichian (INSA-Lyon \& Creatis, France); Sébastien Valette (CNRS UMR520, France); Michel Desvignes (GRENOBLE-INP, France); Remy Prost (CREATIS,; UMR CNRS 5220; Inserm U630;INSA lyon, France) pp. 2149-2152

\section{Cellsnake: a New Active Contour Technique for cell/fibre Segmentation}

Kangyu Pan (Trinity College Dublin, Ireland); Anil Kokaram (Trinity College Dublin, Ireland); Kerry Gilmore (University of Wollongong, Australia); Michael Higgins (University of Wollongong, Australia); Robert Kapsa (University of Wollongong, Australia); Gordon Wallace (University of Wollongong, Australia) pp. 2153-2156

\section{Variational Approach for Segmentation of Lung Nodules}

Amal Farag (University of Louisville, USA); Hossam Abdelmunim (Faculty of Engineering, Ain Shams University, ? ); James Graham (University of Louisville, USA); Aly Farag (University of Louisville, USA); Salwa Elshazly (University of Louisville, USA); Sabry Al Mogy (Mansoura University, Egypt); Mohamed S. Al Mogy (Mansoura Scan Center, Egypt); Robert Falk (Jewish Hospital and 3DR, USA); Sahar Al-Jafarye (University of Louisville, USA); Hani Mahdi (Ain Shams University, Egypt); Rebecca Milam (University of Louisville, USA) pp. 2157-2160

A Novel Probabilistic Simultaneous Segmentation and Registration Using Level Set Melih Aslan (CVIP Lab, University of Louisville, USA); Eslam Mostafa (University of Louisville, USA); Hossam Abdelmunim (Faculty of Engineering, Ain Shams University, ? ); Ahmed Shalaby (University of Louisville, USA); Aly Farag (University of Louisville, USA); Ben Arnold (Image Analysis, Inc., USA) pp. 2161-2164

\section{Efficient Cell Segmentation and Tracking of Developing Plant Meristem}

Katya Mkrtchyan (University of California, Riverside, USA); Damanpreet Singh (UCI, USA); Min Liu (UCR, USA); Venugopala Reddy (UCR, USA); Amit Roy-Chowdhury (University of California, Riverside, USA); Gopi M. (University of California, Irvine, USA)

pp. $2165-2168$ 
Retinal Image Registration Using Bifurcation Structures

Li Chen (Wuhan University of Science and Technology, P.R. China); Yang Xiang (Wuhan University of Science and Technology, P.R. China); YaoJie Chen (Wuhan University of Science and Technology, P.R. China); XiaoLong Zhang (Wuhan University of Science and Technology, P.R. China) pp. 2169-2172

Lung Tumor Delineation in PET-CT Images Using a Downhill Region Growing and a Gaussian Mixture Model

Cherry Ballangan (University of Sydney, Australia); Xiuying Wang (University of Sydney, Australia); Michael Fulham (University of Sydney, Australia); Stefan Eberl (Royal Prince Alfred Hospital, Australia); Dagan Feng (The University of Sydney, Australia) pp. 2173-2176

Temporal Registration of Partial Data Using Particle Filtering Guy Nir (University of British Columbia, Canada); Allen Tannenbaum (Georgia Institute of Technology, USA) pp. $2177-2180$

Markov-Gibbs Model Based Registration of CT Lung Images Using Subsampling for the Follow-Up Assessment of Pleural Thickenings

Peter Faltin (RWTH Aachen University, Germany); Kraisorn Chaisaowong (RWTH Aachen University, Germany); Thomas Kraus (University Hospital Aachen, Germany); Til Aach (RWTH Aachen University, Germany) pp. 2181-2184

Ultrasound-based Surgical Navigation for Percutaneous Renal Intervention: In Vivo Measurements and in Vitro Assessment Zhicheng Li (Shenzhen Institutes of Advanced Technology, Chinese Academy of Science, P.R. China); Jia Gu (Shenzhen Institutes of Advanced Technology, Chinese Academy of Science, P.R. China); Jacob Chakareski (EPFL, Switzerland); Lei Wang (Chinese Academy of Sciences, P.R. China) pp. 2185-2188

Learning Shape Statistics for Hierarchical 3D Medical Image Segmentation Wuxia Zhang (Chinese Academy of Sciences, P.R. China); Yuan Yuan (Chinese Academy of Sciences, P.R. China); Xuelong Li (Xi'an Institute of Optics and Precision Mechanics, Chinese Academy of Sciences, P.R. China); Pingkun Yan (Chinese Academy of Sciences, P.R. China) pp. 2189-2192

\section{TP.PC: Image/Video Coding, Streaming and Recovery (Poster)}

\section{HTTP-Based Scalable Video Streaming Over Mobile Networks}

Ktawut Tappayuthpijarn (Technical University of Munich \& Nomor Research GmbH, Germany); Thomas Stockhammer (Nomor Research GmbH, Germany); Eckehard Steinbach (Munich University of Technology, Germany) pp. 2193-2196 
Joint Source-Channel Coding Optimization with Packet Loss Resilience for Video Transmission

Ching-Hui Chen (Academia Sinica, Taiwan); Wei-Ho Chung (Academia Sinica, Taiwan); Yu-Chiang Frank Wang (Academia Sinica, Taiwan) pp. $2197-2200$

Joint Space-Time-View Error Concealment Algorithms for 3D Multi-View Video Walid EI Shafai (E-JUST University, Egypt); Branislav Hrušovský (Technical University of Košice, Slovakia); Mostafa El-Khamy (Egypt-Japan University of Science and Technology \& Alexandria University, Faculty of Engineering, Egypt); Mohamed ElSharkawy (Purdue School of Engineering and Technology, USA) pp. 2201-2204

\section{Expanding Window Random Linear Codes for Data Partitioned H.264 Video}

Transmission Over DVB-H Network

Sajid Nazir (University of Strathclyde, United Kingdom); Vladimir Stankovic (University of Strathclyde, United Kingdom); Dejan Vukobratović (University of Novi Sad, Serbia) pp. 2205-2208

Temporal Adaptation Strategies for Spatio-Temporal Image Alignment in Inter-

Sequence Error Concealment of Digital TV

Tobias Tröger (University of Erlangen-Nuremberg, Germany); Andre Kaup (University of Erlangen-Nuremberg, Germany)

pp. 2209-2212

An Improved Error Concealment by Diminishing the Edge Discontinuity Jun-Horng Chen (Oriental Institute of Technology, Taiwan)

pp. 2213-2216

High-Frequency Error Recovery in JPEG XR Coded Images

Cristian Perra (University of Cagliari, Italy)

pp. $2217-2220$

Joint Source-Channel Decoding of Motion-Information Using Maximum-a-Posteriori Ângelo Arrifano (I3S-CNRS-University of Nice Sophia Antipolis \& University of Beira Interior, Portugal); Marc Antonini (I3S-CNRS-University of Nice Sophia Antipolis, France); Manuela Pereira (University of Beira Interior, Portugal); Mario M. Freire (University of Beira Interior, Portugal)

pp. 2221-2224

New TCP Video Streaming Proxy Design for Last-Hop Wireless Networks Wei Pu (State University of New York at Buffalo, USA); Zixuan Zou (Huawei Technologies Co. LTD, P.R. China); Chang Wen Chen (State University of New York at Buffalo, USA)

pp. 2225-2228

Pixel Domain Referenceless Visual Degradation Detection and Error Concealment for Mobile Video

Luc Trudeau (École de Technologie Supérieure, Université du Québec, Canada); Stephane Coulombe (Ecole de technologie supérieure, Canada); Steven Pigeon (Ecole de technologie supérieure, Canada)

pp. 2229-2232 
Contribution-based Peer Selection for Packet Protection for P2P Video Streaming
Over Mesh-based Networks

Chi-Wen Lo (National Tsing Hua University, Taiwan); Chia-Wen Lin (National Tsing Hua University, Taiwan); Yung-Chang Chen (National Tsing Hua University, Taiwan); Yu Jen-Yu (Industrial Technology Research Institute, Taiwan) pp. 2233-2236

\section{Channel Protection for H.264 Compression in Transportation Video Surveillance Applications}

Eren Soyak (Northwestern University, USA); Sotirios A. Tsaftaris (Northwestern University, USA); Aggelos K. Katsaggelos (Northwestern University, USA) pp. $2237-2240$

Scalable Video Streaming Over OpenFlow Networks:An Optimization Framework for QoS Routing

Hilmi Enes Egilmez (Koc University, Turkey); Burak Gorkemli (Koç University, Turkey); A. Murat Tekalp (Koc University, Turkey); Seyhan Civanlar (Argela Technologies, Turkey)

pp. 2241-2244

\section{Error Recovery of Image-Based Depth Maps Using Bézier Curve Fitting} Sylvain Marcelino (Universidade de Tras-os-Montes e Alto Douro / Instituto de Telecomunicacoes Leiria, Portugal); Pedro A. Amado Assuncao (Polytechnic Institute of Leiria / Instituto de Telecomunicacoes, Portugal); Sérgio M. M. Faria (Institute of Telecommunications \& Polytechnic Institute of Leiria, Portugal); Salviano Soares (Universidade de Trás-os-Montes e Alto Douro, Portugal) pp. 2245-2248

Adaptive Policies for Real-Time Video Transmission: A Markov Decision Process Framework

Chao Chen (The University of Texas at Austin, USA); Robert Heath (The University of Texas at Austin, USA); Alan C Bovik (University of Texas at Austin, USA); Gustavo de Veciana (The University of Texas at Austin, USA) pp. 2249-2252

Adaptive Frame and QP Selection for Temporally Super-Resolved Full-Exposure-Time Video

Mlhoko Shimano (University of Tokyo, Japan); Gene Cheung (National Institute of Informatics, Japan); Imari Sato (National Institute of Informatics, Japan) pp. 2253-2256

\section{TP.PD: Computational and Magnetic Resonance Imaging (Poster)}

\section{An Exploration Framework for Segmentation Parameter Spaces}

Sarra Ben Fredj (Creatis, France); Tristan Glatard (University of Lyon ; CREATISLRMN, France); Christopher Casta (CREATIS, France); Patrick Clarysse (CREATISLRMN, CNRS UMR 5220, INSERM U630, France) pp. 2257-2260

\section{Parallel Quadratic Programming for Image Processing} Matthew Brand (MERL, USA); Donghui Chen (Tufts University, USA) pp. 2261-2264 
Low Visual Difference Virtual High Dynamic Range Image Synthesizer From a Single Legacy Image

Tsun-Hsien Wang (National Tsing Hua University, Taiwan); Ching-Te Chiu (National

Tsing Hua University, Taiwan)

pp. 2265-2268

Towards a Diffusion Image Processing Validation and Accuracy Prediction Framework Francesca Pizzorni Ferrarese (University of Verona, Italy); Alessandro Daducci (École Polytechnique Fédérale de Lausanne, Switzerland); Meritxell Bach Cuadra (Ecole Polytechnique Fédérale de Lausanne (EPFL), Switzerland); Alia Lemkaddem (École Polytechnique Fédérale de Lausanne, Switzerland); Cristina Granziera (École Polytechnique Fédérale de Lausanne, Switzerland); Jean-Philippe Thiran (École Polytechnique Fédérale de Lausanne \& Signal Processing Laboratory, Switzerland); Gloria Menegaz (University of Verona, Italy) pp. 2269-2272

\section{A New Similarity Measure for Multi-Modal Image Registration} Mark Pickering (UNSW@adfa, Australia) pp. 2273-2276

\section{Segmented Rapid Magnetic Resonance Imaging Using Structured Sparse}

Representations

Vimal Singh (University of Texas, Austin, USA); Dan Wang (University of Texas, Austin, USA); Ahmed Tewfik (University of Texas, Austin, USA) pp. $2277-2280$

\section{Extended Kalman Filtering for MR-thermometry Guided High Intensity Focused}

\section{Ultrasound Using the Bio Heat Transfer Equation}

Sébastien Roujol (University of Bordeaux 2, France); Baudouin Denis de Senneville (University of Bordeaux 2, France); Silke Hey (University of Bordeaux 2, France); Chrit Moonen (University of Bordeaux, France); Mario Ries (Laboratory for Molecular and Functional Imaging, France) pp. 2281-2284

3D Automatic Approach For Precise Segmentation of the Prostate From DiffusionWeighted Magnetic Resonance Imaging

Ahmad Firjani (University of Louisville, USA); Fahmi Khalifa (Bioimaging Laboratory \& University of Louisville, Louisville, KY, USA); Ahmed Elnakib (Bioimaging Lab, USA); Georgy Gimel'farb (University of Auckland, USA); Mohamed Abo El-Ghar (University of Mansoura, Egypt); Adel S Elmaghraby (University of Louisville, USA); Ayman Sabry El-Baz, PhD (University of Louisville, USA) pp. 2285-2288

A New Framework for Automated Segmentation of Left Ventricle Wall From Contrast Enhanced Cardiac Magnetic Resonance Images Ahmed Elnakib (Bioimaging Lab, USA); Garth Beache (Diagnostic Radiology Department, USA); Georgy Gimel'farb (University of Auckland, USA); Ayman Sabry El-Baz, PhD (University of Louisville, USA) pp. 2289-2292

Dynamic Compressive Magnetic Resonance Imaging Using a Gaussian Scale Mixtures Model

Yookyung Kim (University of Arizona, USA); Mariappan Nadar (Siemens Corporation, Corporate Research, USA); Ali Bilgin (ECE Dept, The University of Arizona, USA) 
pp. $2293-2296$

Orthonormal Expansion lell_1-Minimization for Compressed Sensing in MRI Jun Deng (Nanyang Technological University, Singapore); Zai Yang (Nanyang Technological University, Singapore); Cishen Zhang (Swinburne University of Technology, Australia); Lu Wenmiao (Nanyang Technological University, Singapore) pp. 2297-2300

\section{TP.PE: Tracking and Motion Detection (Poster)}

\section{Human Pose Tracking in Low Dimensional Space Enhanced by Limb Correction} Alexandros Moutzouris (Kingston University, United Kingdom); Jesus Martinez-delRincon (Kingston University, United Kingdom); Michal Lewandowski (Kingston University, United Kingdom); Jean-Christophe Nebel (Kingston University, United Kingdom); Dimitrios Makris (Kingston University, United Kingdom) pp. 2301-2304

\section{Human Tracking by Structured Body Parts}

Yingkun Xu (Chinese Academy of Sciences \& Institute of Computing Technology, P.R. China); Lei Qin (Institute of Computing Tech, Chinese Academy of Science, P.R. China); Shuqiang Jiang (Institute of Computing Technology, Chinese Academy of Sciences, Beijing, P.R. China); Qingming Huang (Graduate School of Chinese Academy of Sciences, P.R. China) pp. 2305-2308

Real-Time Moving Object Segmentation and Tracking for H.264/AVC Surveillance Videos

Pei Dong (University of Sydney \& Beijing University of Technology, Australia); Yong Xia (University of Sydney, Australia); Zhuo Li (Beijing University of Technology, P.R. China); Dagan Feng (The University of Sydney, Australia) pp. 2309-2312

Tracking Pedestrians Using Smoothed Colour Histograms in an Interacting Multiple Model Framework

Zhengqiang Jiang (The University of Western Australia, Australia); Du Huynh (The University of Western Australia, Australia); Bill Moran (University of Melbourne, Australia); Subhash Challa (The University of Melbourne, Australia) pp. 2313-2316

Contour Tracking Via On-line Discriminative Appearance Modeling Based Level Sets Xin Sun (Harbin Institute of Technology, P.R. China); Hongxun Yao (Harbin Institute of Technology, P.R. China); Shengping Zhang (Harbin Institute of Technology, P.R. China) pp. 2317-2320

Lip Contour Tracking Using Multiple Dynamic Models on a Manifold Jacinto C. Nascimento (Instituto de Sistemas e Robotica, Portugal); Jorge Silva (Duke University, USA)

pp. 2321-2324

\section{PFT: a Protocol for Evaluating Video Trackers}

Tahir Nawaz (Queen Mary, University of London, United Kingdom); Andrea Cavallaro (Queen Mary, University of London, United Kingdom) 
pp. $2325-2328$

Real-time Human Tracking Based on Switching Linear Dynamic System Combined with Adaptive Meanshift Tracker

Zheyuan Li (Peking University, P.R. China); Hong Liu (Peking University, P.R. China); Chao Xu (Peking University, P.R. China)

pp. 2329-2332

A Structured Learning-based Graph Matching for Dynamic Multiple Object Tracking Dayu Zheng (Shanghai Jiao Tong University, P.R. China); Hongkai Xiong (Shanghai Jiao Tong University, P.R. China); Yuan F. Zheng (Ohio State University, USA) pp. 2333-2336

Motion Detection in Old Film Sequences Using Adaptive Gaussian Mixture Model Xiaoyong Zhang (Tohoku University, Japan); Masahide Abe (Tohoku University, Japan); Masayuki Kawamata (Tohoku University, Japan) pp. 2337-2340

Gradient Sparsity for Piecewise Continuous Optical Flow Estimation Junyu Han (Xidian University, P.R. China); Fei Qi (Xidian University, P.R. China); Guangming Shi (Xidian University, P.R. China) pp. 2341-2344

Efficient Real-Time Local Optical Flow Estimation by Means of Integral Projections Tobias Senst (Technische Universität Berlin, Germany); Volker Eiselein (Technische Universität Berlin, Germany); Michael Pätzold (Technische Universität Berlin, Germany); Thomas Sikora (Technische Universität Berlin, Germany) pp. $2345-2348$

Video Motion Detection Algorithm Using Probabilistic Time Integrated Ransac Tal Nir (Rafael, Israel); Orit Eden (Rafael, Israel) pp. 2349-2352

Identifying Salient Poses in Lecture Videos John R Zhang (Columbia University, USA); John R. Kender (Columbia University, USA) pp. 2353-2356

Feature Selection with Geometric Constraints for Vision-Based Unmanned Aerial Vehicle Navigation

Maria E. Angelopoulou (Imperial College London, United Kingdom); Christos-Savvas Bouganis (Imperial College London, United Kingdom) pp. 2357-2360

\section{TP.PF: Scene Analysis (Poster)}

Two-phase Approach for Multi-view Object Extraction

Sungheum Kim (KAIST, Korea); Yu-wing Tai (KAIST, Korea); Yunsu Bok (KAIST, Korea); Hyeongwoo Kim (KAIST, Korea); In-So Kweon (Korea Advanced Institute of Science and Technology (KAIST), Korea) pp. 2361-2364 
Alignment of Uncalibrated Images for Multi-View Classification

Sercan Ömer Arık (Bilkent University, Turkey); Elif Vural (Ecole Polytechnique Federale de Lausanne, Switzerland); Pascal Frossard (Swiss Federal Institute of Technology - EPFL, Switzerland) pp. 2365-2368

Dynamic Background Subtraction Using Moments Romain Marie (MIS, France); Alexis Potelle (MIS, France); El Mustapha Mouaddib (MIS, France) pp. 2369-2372

Belief Propagation with Local Edge Detection-based Cost Aggregation for Stereo Matching

Fu He (Southeast University, P.R. China); Feipeng Da (Southeast University, P.R. China)

pp. 2373-2376

Discrimination and Description of Repetitive Patterns for Enhancing Object

Recognition Performance

Seong Jong Ha (Seoul National University, Korea); Sang Hwa Lee (Seoul National University, Korea); Nam-Ik Cho (Seoul National University, Korea)

pp. 2377-2380

\section{Sparse Regression Analysis for Object Recognition}

Baochang Zhang (Beihang University, P.R. China); Shengping Zhang (Harbin Institute of Technology, P.R. China); Jianzhuang Liu (The Chinese University of Hong Kong, Hong Kong)

pp. 2381-2384

Intelligent Filtering by Semantic Importance for Single-View 3D Reconstruction From Snooker Video

Philip Legg (Swansea University, United Kingdom); Matthew Parry (Swansea University, United Kingdom); David Chung (Swansea University, United Kingdom); Richard M. Jiang (Swansea University, United Kingdom); Adrian Morris (Swansea University, United Kingdom); Iwan Griffiths (Swansea University, United Kingdom); David Marshall (Cardiff University, United Kingdom); Min Chen (Swansea University, United Kingdom) pp. 2385-2388

Pattern Recognition Using Rotation-invariant Filter-driven Template Matching Yi-Chong Zeng (Academia Sinica, Taiwan) pp. 2389-2392

3D Spatio-temporal Graph Cuts for Video Objects Segmentation Zhiqiang Tian (Xi'an Jiaotong University, P.R. China); Jianru Xue (Xi'an Jiaotong University, P.R. China); Nanning Zheng (Xi'an Jiaotong University, P.R. China); Xuguang Lan (Xi'an Jiaotong University \& Institute of Artificial Intelligence and Robotics, P.R. China); Ce Li (Xi'an Jiaotong University, P.R. China) pp. 2393-2396

Hierarchical Invariant Sparse Modeling for Image Analysis Leah Bar (Tel Aviv University, Israel); Guillermo Sapiro (University of Minnessotta, USA) pp. $2397-2400$ 
Multi-scale Analysis of Color and Texture for Salient Object Detection Ketan Tang (Hong Kong University of Science and Technology, Hong Kong); Oscar C. Au (HKUST, Hong Kong); Lu Fang (Hong Kong University of Science and Technology, Hong Kong); Zhiding Yu (Hong Kong University of Science and Technology \& Shenzhen Institutes of Advanced Technology, Chinese Academy of Sciences, Hong Kong); Yuanfang Guo (Hong Kong University of Science and Technology, Hong Kong) pp. 2401-2404

Object Detection Using Discriminative Photogrammetric Context Yuanliu Liu (Xi'an Jiaotong University, P.R. China) pp. 2405-2408

\section{Severity Classification of Abnormal Traffic Events At Intersections} Omer Aköz (Yildiz Technical University, Turkey); Elif Karslıgil (Yildiz Technical University, Turkey)

pp. 2409-2412

Soft Assignment of Visual Words as Linear Coordinate Coding and Optimisation of Its Reconstruction Error

Piotr Koniusz (University of Surrey, United Kingdom); Krystian Mikolajczyk (University of Surrey, United Kingdom)

pp. 2413-2416

One Step Beyond Bags of Features: Visual Categorization Using Components Jing Liu (Institute of Automation, Chinese Acadamy of Sciences, P.R. China); Chunjie Zhang (Institute of Automation, Chinese Academy of Sciences, P.R. China); Qi Tian (University of Texas at San Antonio, USA); Changsheng Xu (Institute of Automation, Chinese Academy of Sciences \& China-Singapore Institute of Digial Media, P.R. China); Hanqing Lu (the Institute of Automation, Chinese Academy of Sciences, P.R. China); Songde Ma (Institute of Automation, Chinese Academy of Sciences, P.R. China) pp. $2417-2420$

Linear SVM Classification Using Boosting HOG Features for Vehicle Detection in LowAltitude Airborne Videos

Xianbin Cao (Beihang University, P.R. China); Changxia Wu (University of Science and Technology of China, P.R. China); Pingkun Yan (Chinese Academy of Sciences, P.R. China); Xuelong Li (Xi'an Institute of Optics and Precision Mechanics, Chinese Academy of Sciences, P.R. China) pp. 2421-2424

TP.PG: Image Retrieval (Poster)

\section{Semantic Clusters Based Manifold Ranking for Image Retrieval} Ran Chang (Utah State University, USA); Xiaojun Qi (Utah State University, USA) pp. $2425-2428$

Browsing Catalogue Graphs: Content Caching Supercharged!! Jacob Chakareski (EPFL, Switzerland) pp. 2429-2432 
MPEG-7 Compliant Generalized Structure Descriptor for Still Image Indexing Constantin Vertan (University Politehnica of Bucharest, Romania); Marta Zamfir (Tessera Technologies, Inc., Romania); Alexandru Drîmbărean (Tessera Ireland, Ireland); Adrian Zamfir (Tessera Technologies, Inc., Romania) pp. 2433-2436

Key Frame Extraction From Consumer Videos Using Sparse Representation Mrityunjay Kumar (Eastman Kodak Company, USA); Alexander Loui (Eastman Kodak Company, USA) pp. $2437-2440$

On the Use of Conceptual Information in a Concept-Based Image Indexing and Retrieval Framework

Radi Jarrar (MONASH University, Malaysia); Mohammed Belkhatir (CNRS Lyon, France); Chris Messom (MONASH University, Malaysia) pp. 2441-2444

Improving Image Tag Recommendation Using Favorite Image Context Wonyong Eom (Korea Advanced Institute of Science and Technology, Korea); Sihyoung Lee (Korea Advanced Institute of Science and Technology, Korea); Wesley Marcel De Neve (Korea Advanced Institute of Science and Technology (KAIST), Korea); Yong Man Ro (KAIST, Korea) pp. 2445-2448

Query Sensitive Dynamic Web Video Thumbnail Generation Chunxi Liu (Graduate University of Chinese Academy of Sciences, CAS, P.R. China); Qingming Huang (Graduate School of Chinese Academy of Sciences, P.R. China); Shuqiang Jiang (Institute of Computing Technology, Chinese Academy of Sciences, Beijing, P.R. China) pp. 2449-2452

Graph-Based Multiple-Instance Learning with Instance Weighting for Image Retrieval Fei Li (Fujitsu Research and Development Center Co., Ltd., P.R. China); Rujie Liu (Fujitsu Research \& Development Co., Ltd, P.R. China) pp. 2453-2456

Image Database Categorization Using Robust Unsupervised Learning of Finite Generalized Dirichlet Mixture Models Mohamed Ben Ismail (University of Louisville, USA); Hichem Frigui (University of Louisville, USA) pp. $2457-2460$

Weakly Supervised Locality Sensitive Hashing for Duplicate Image Retrieval Cao Yudong (Beijing University of Posts and Telecommunications, P.R. China); Zhang Honggang (Beijing University of Posts and Telecommunications, P.R. China); Jun Guo (BUPT, P.R. China)

pp. 2461-2464

\section{Fast Common Visual Pattern Detection Via Radiate Geometric Model}

Lingyang Chu (Institute of Computing Technology, Chinese Academy of Science, P.R. China); Shuqiang Jiang (Institute of Computing Technology, Chinese Academy of Sciences, Beijing, P.R. China); Qingming Huang (Graduate School of Chinese Academy of Sciences, P.R. China)

pp. 2465-2468 
A Novel Image Importance Model for Content-Aware Image Resizing

Wonjun Kim (Korea Advanced Institute of Science and Technology (KAIST), Korea) pp. 2469-2472

Multi-Keyframe Abstraction From Videos

Ping Li (The Chinese University of Hong Kong, Hong Kong); Yanwen Guo (Nanjing University, P.R. China); H. Q. Sun (The Chinese University of Hong Kong, P.R. China) pp. 2473-2476

Select Informative Features for Recognition

Zixuan Wang (Stanford University, USA)

pp. $2477-2480$

\section{A Balanced Semi-supervised Hashing Method for CBIR}

Jianhui Zhou (Dalian University of Technology, P.R. China); Haiyan Fu (Dalian University of Technology, P.R. China); Xiangwei Kong (Dalian University of Technology, P.R. China)

pp. 2481-2484

\section{Wednesday, September 14}

\section{WA.L1: Recent Advances in Web-scale Image Annotation (Special Session)}

\section{Learning the Trip Suggestion From Landmark Photos on the Web}

Rongrong Ji (Columbia University, P.R. China); Ling-Yu Duan (Peking University, P.R. China); Jie Chen (Peking University, P.R. China); Shuang Yang (Peking University, P.R. China); Hongxun Yao (Harbin Institute of Technology, P.R. China); Tiejun Huang (Peking University, P.R. China); Wen Gao (ICT-ISVISION Joint R\&D Laboratory for Face Recognition, CAS, P.R. China) pp. $2485-2488$

\section{Online Vicept Learning for Web-Scale Image Understanding}

Liang Li (Institute of Computing Technology, Chinese Academy of Sciences, P.R. China); Shuqiang Jiang (Institute of Computing Technology, Chinese Academy of Sciences, Beijing, P.R. China); Qingming Huang (Graduate School of Chinese Academy of Sciences, P.R. China) pp. 2489-2492

break

Representative Sampling with Certainty Propagation for Image Retrieval Jian Cheng (Chinese Academy of Sciences, P.R. China); Biao Niu (Institute of Automation, Chinese Academy of Sciences, P.R. China); Hanqing Lu (the Institute of Automation, Chinese Academy of Sciences, P.R. China) pp. 2493-2496

\section{Learning Semantic Embedding At a Large Scale}

Min-Hsuan Tsai (University of Illinois at Urbana-Champaign, USA); Jinjun Wang (Epson Research and Development, USA); Tong Zhang (Rutgers University, USA); 
Yihong Gong (NEC Labs American, USA); Thomas S Huang (University of Illinois at Urbana-Champaign, USA)

pp. 2497-2500

\section{Descriptive Local Feature Groups for Image Classification}

Lei Yu (Institute of Automation?Chinese Academy of Sciences, P.R. China); Jing Liu (Institute of Automation, Chinese Acadamy of Sciences, P.R. China); Changsheng Xu (Institute of Automation, Chinese Academy of Sciences \& China-Singapore Institute of Digial Media, P.R. China)

pp. 2501-2504

\section{WA.L2: Video Quality Assessment (Lecture)}

\section{A Spatiotemporal Most-Apparent-Distortion Model for Video Quality Assessment}

Phong Vu (Oklahoma State University, USA); Cuong Vu (Oklahoma State University, USA); Damon Chandler (Oklahoma State University, USA)

pp. 2505-2508

\section{Spatio-temporal Quality Pooling Accounting for Transient Severe Impairments and Egomotion \\ Jin. C. Park (Yonsei University, Korea); Kalpana Seshadrinathan (Intel, USA); \\ Sanghoon Lee (Yonsei University, Korea); Alan C Bovik (University of Texas at Austin, USA) \\ pp. 2509-2512}

break

Machine Learning Based Modeling of Spatial and Temporal Factors for Video Quality Assessment

Manish Narwaria (Nanyang Technological University, Singapore); Weisi Lin (Nanyang Technological University, Singapore)

pp. 2513-2516

\section{A Novel Full-Reference Video Quality Metric and Its Application to Wireless Video} Transmission

Yang Peng (Technische Universität München, Germany); Eckehard Steinbach (Munich University of Technology, Germany)

pp. $2517-2520$

\section{D-DCT Based Perceptual Quality Assessment of Stereo Video}

Lina Jin (Tampere University of Technology, Finland); Atanas Boev (Tampere University of Technology, Finland); Atanas Gotchev (Tampere University of Technology, Finland); Karen Egiazarian (Tampere University of Technology, Finland) pp. 2521-2524

A Perceptual Quality Assessment Metric Using Temporal Complexity and Disparity Information for Stereoscopic Video Munchurl Kim (Korea Advanced Institute of Science and Technology, Korea) pp. 2525-2528 


\section{WA.L3: Copy and Near-Duplicate Detection (Lecture)}

\section{Commercial Mining Based on Temporal Recurrence Hashing Algorithm and Bag-Of- Fingerprints Model}

Xiaomeng Wu (National Institute of Informatics, Japan); Shin'ichi Satoh (National Institute of Informatics, Japan)

pp. 2529-2532

\section{Copy Detection Towards Semantic Mining for Video Retrieval}

Shikui Wei (Beijing Jiaotong University \& Nanyang Technological University, P.R. China); Yao Zhao (Beijing Jiaotong University, P.R. China); Changsheng Xu (Institute of Automation, Chinese Academy of Sciences \& China-Singapore Institute of Digial Media, P.R. China); Xu Dong (Powerlayer Microsystems, P.R. China)

pp. 2533-2536

break

\section{Salient Covariance for Near-duplicate Image and Video Detection}

Ligang Zheng (Sun Yat-sen University, P.R. China); Guoping Qiu (University of Nottingham, United Kingdom); Jiwu Huang (Sun Yat-sen University, P.R. China); Hao Fu (University of Nottingham, United Kingdom) pp. $2537-2540$

\section{Shape Context Based Image Hashing Using Local Feature Points}

Xudong Lv (University of British Columbia, Canada); Z. Jane Wang (University of British Columbia, Canada) pp. 2541-2544

\section{PKUBench: A Context Rich Mobile Visual Search Benchmark}

Rongrong Ji (Columbia University, P.R. China); Ling-Yu Duan (Peking University, P.R. China); Jie Chen (Peking University, P.R. China); Shuang Yang (Peking University, P.R. China); Tiejun Huang (Peking University, P.R. China); Hongxun Yao (Harbin Institute of Technology, P.R. China); Wen Gao (ICT-ISVISION Joint R\&D Laboratory for Face Recognition, CAS, P.R. China) pp. 2545-2548

\section{Fast Face Sequence Matching in Large-scale Video Databases}

Hung Thanh Vu (University of Science \& University of Science, Ho Chi Minh city, Vietnam); Thanh Duc Ngo (The Graduate University for Advanced Studies, Japan); Thao-Ngoc Nguyen (University of Science, Vietnam); Duy-Dinh Le (National Institute of Informatics, Japan); Shin'ichi Satoh (National Institute of Informatics, Japan); Le Bac (University of Science, Vietnam); Anh Duc Duong (University of Science, VNUHCM, Vietnam)

pp. 2549-2552

\section{WA.L4: Image Denoising (Lecture)}

\section{Patch-based Locally Optimal Denoising}

Priyam Chatterjee (Pelican Imaging Corporation, USA); Peyman Milanfar (University of California, Santa Cruz, USA)

pp. 2553-2556 


\section{Nonlinear Curvelet Diffusion for Noisy Image Enhancement}

Ying Li (Northwestern Polytechnical University, P.R. China); Huijun Ning (Northwestern Polytechnical University, P.R. China); Yanning Zhang (Northwestern Polytechnical University, P.R. China); David Dagan Feng (University of Sydney \& Hong Kong Polytechnic University, Australia) pp. 2557-2560

break

MMSE Nonlocal Means Denoising Algorithm for Poisson Noise Removal Chul Lee (Korea University, Korea); Chulwoo Lee (Korea University, Korea); ChangSu Kim (Korea University, Korea) pp. 2561-2564

\section{Learning a Wavelet Tree for Multichannel Image Denoising}

Zhen James Xiang (Princeton University, USA); Zhuo Zhang (Princeton University, USA); Pingmei Xu (Princeton University, USA); Peter Ramadge (Princeton University, USA)

pp. 2565-2568

\section{Robust Sparse Image Denoising}

Radovan Obradovic (RT-RK Novi Sad, Serbia); Marko Janev (Mathematical Institute of the Serbian Academy of Sciences and Arts, Serbia); Borislav Antic (University of Heidelberg, Germany); Vladimir Crnojević (Novi Sad, Serbia); Nemanja Petrovic (University of Novi Sad, Serbia) pp. 2569-2572

Video Denoising Based on Transform Domain Minimum Mean Square Error Dai (Hong Kong University of Science and Technology, Hong Kong); Oscar C. Au (HKUST, Hong Kong); Chao Pang (Hong Kong University of Science and Technology, Hong Kong); Feng Zou (Hong Kong University of Science and Technology, Hong Kong)

pp. 2573-2576

\section{WA.L5: 3D Video Processing and Rendering (Lecture)}

\section{Scribble Based Interactive 3D Reconstruction Via Scene Co-segmentation} Adarsh Kowdle (Cornell University, USA); Yao-Jen Chang (Cornell University, USA); Dhruv Batra (Carnegie Mellon University, USA); Tsuhan Chen (Cornell University, USA)

pp. $2577-2580$

\section{Adaptive Plenoptic Sampling}

Christopher Gilliam (Imperial College London, United Kingdom); Pier Luigi Dragotti (Imperial College London, United Kingdom); Mike Brookes (Imperial College London, United Kingdom)

pp. 2581-2584

break 


\section{Efficient Depth Blurring with Occlusion Handling}

Timothy Popkin (Vision Semantics Ltd \& Queen Mary University of London, United Kingdom); Andrea Cavallaro (Queen Mary, University of London, United Kingdom); David Hands (British Telecomunnications plc, United Kingdom) pp. $2585-2588$

\section{A New Multidirectional Extrapolation Hole-Filling Method for Depth-Image-Based Rendering}

Lai Man Po (City University of Hong Kong, Hong Kong); Shihang Zhang (Shenzhen Graduate School of Peking University, P.R. China); Xuyuan Xu (City University of Hong Kong, Hong Kong); Yuesheng Zhu (Shenzhen Graduate School, Peking University, P.R. China)

pp. 2589-2592

\section{Parameterization and Appearance Preserving on Cubic Cells for 3D Digital}

Preservation of Cultural Heritage Karl Apaza-Agüero (Universidade Federal do Parana, Brazil); Luciano Silva (Universidade Federal do Paraná, Brazil); Olga R Bellon (Universidade Federal do Parana \& IMAGO Research Group, Brazil) pp. 2593-2596

\section{Can 3D Synthesized Views Be Reliably Assessed Through Usual Subjective and} Objective Evaluation Protocols?

Emilie Bosc (Institut National des Sciences Appliquées de Rennes (INSA de Rennes), France); Martin Köppel (Fraunhofer Institut for Telecomunications, Heinrich-HertzInstitut, Germany); Romuald Pépion (IRCCyN, Université de Nantes, France); Muriel Pressigout (IETR / INSA de Rennes, France); Luce Morin (INSA-Rennes, France); Patrick Ndjiki-Nya (Fraunhofer-Gesellschaft, Germany); Patrick Le Callet (IRCCYN, France) pp. $2597-2600$

\section{WA.L6: Exploiting and Analyzing Text in Electronic Images (Lecture)}

Mobile Visual Search on Printed Documents Using Text and Low Bit-Rate Features Sam S Tsai (Stanford University, USA); Huizhong Chen (Stanford University, USA); David M Chen (Stanford University, USA); Georg Schroth (Technische Universität München, Germany); Radek Grzeszczuk (Nokia Research Center, USA); Bernd Girod (Stanford University, USA) pp. 2601-2604

\section{A New Hybrid Method to Detect Text in Natural Scene} Gang Zhou (Xi'an Jiaotong University, P.R. China); Yuehu Liu (Xi'an Jiaotong University, P.R. China); Zhiqiang Tian (Xi'an Jiaotong University, P.R. China); Yuanqi Su (Xi'an Jiaotong University, P.R. China) pp. 2605-2608

break

Robust Text Detection in Natural Images with Edge-enhanced Maximally Stable Extremal Regions

Huizhong Chen (Stanford University, USA); Sam S Tsai (Stanford University, USA); Georg Schroth (Technische Universität München, Germany); David M Chen (Stanford 
University, USA); Radek Grzeszczuk (Nokia Research Center, USA); Bernd Girod (Stanford University, USA)

pp. 2609-2612

Handwritten Connected Digits Detection: An Approach Using Instance Selection Cristiano Pereira (Federal University of Pernambuco \& Federal Institute of

Pernambuco, Brazil); George D. C. Cavalcanti (Federal University of Pernambuco, Brazil)

pp. 2613-2616

A Robust Skew Detection Method Based on Maximum Gradient Difference and Rsignature

Mehdi Felhi (University Nancy 2 \& Océ - Canon Group, France); Nicolas Bonnier (Océ - Canon Group, France); Salvatore Tabbone (University Nancy 2, France) pp. 2617-2620

\section{Automated Image Quality Assessment for Camera-Captured OCR}

Xujun Peng (BBN Technologies, USA); Huaigu Cao (Raytheon BBN Technologies, USA); Krishna Subramanian (Raytheon BBN Technologies, USA); Rohit Prasad (BBN Technologies, USA); Premkumar Natarajan (BBN Technologies, USA) pp. 2621-2624

\section{WA.L7: Distributed Video Coding (Lecture)}

\section{Progressive Correlation Noise Refinement for Transform Domain Wyner-Ziv Video} Coding

Juan Song (State Key Lab. of Integrated Service Networks, Xidian University, P.R. China); Keyan Wang (State Key Lab. of Integrated Service Networks, Xidian University, P.R. China); Haiying Liu (State Key Lab. of Integrated Service Networks, Xidian University, P.R. China); Yunsong Li (Xidan University, P.R. China); Chengke Wu (State Key Laboratory of ISN, XI'DIAN University, P.R. China) pp. 2625-2628

Global Motion Guided Adaptive Temporal Inter- / Extrapolation for Side Information Generation in Distributed Video Coding

Ralph Hänsel (University of Rostock, Germany); Erika Müller (University of Rostock, Germany)

pp. 2629-2632

break

Parallel Iterative Decoding of Transform Domain Wyner-Ziv Video Using Cross Bitplane Correlation

Huynh Luong (Technical University of Denmark, Denmark); Xin Huang (Technical University of Denmark, Denmark); Soren Forchhammer (Technical University of Denmark, Denmark)

pp. 2633-2636

Improved Wyner-ziv Video Coding Efficiency Using Bit Plane Prediction Jeffrey Micallef (University of Malta, Malta); Reuben A. Farrugia (University of Malta, Malta); Carl J. Debono (University of Malta, Malta)

pp. $2637-2640$ 


\title{
Onboard Low-Complexity Compression of Solar Images
}

Shuang Wang (University of Oklahoma, USA); Lijuan Cui (University of Oklahoma, USA); Samuel Cheng (University of Oklahoma, USA); Lina Stankovic (University of Strathclyde, United Kingdom); Vladimir Stankovic (University of Strathclyde, United Kingdom)

pp. 2641-2644

\section{WA.L8: MRI: Cardiac and Neural Applications (Lecture)}

\author{
A Novel Approach for Accurate Estimation of Left Ventricle Global Indexes From \\ Short-Axis Cine MRI \\ Fahmi Khalifa (Bioimaging Laboratory \& University of Louisville, Louisville, KY, USA); \\ Garth Beache (Diagnostic Radiology Department, USA); Georgy Gimel'farb \\ (University of Auckland, USA); Ayman Sabry El-Baz, PhD (University of Louisville, \\ USA) \\ pp. $2645-2648$
}

Aorta Segmentation Using the Watershed Algorithm for an Augmented Reality System in Laparoscopic Surgery

Fernando López-Mir (Universidad Politécnica de Valencia \& Instituto Interuniversitario de Investigación en Bioingeniería y Tecnología Orientada al ser Humano, Spain); Valery Naranjo (Universidad Politecnica de Valencia, Spain); Jesus Angulo (MINES Paristech, France); Eliseo Villanueva (Universidad Politécnica de Valencia, Spain); Mariano Alcañiz (Universidad Politécnica de Valencia, Spain); Susana López-Celada (Hospital Clínica Benidorm, Unidad Resonancia Magnética, INNSCANER, Spain) pp. 2649-2652

break

Automatic Subcortical Tissue Segmentation of MR Images Using Optimum-Path Forest Clustering

Fábio A. M. Cappabianco (Federal University of São Paulo \& Universidade Federal de Sao Paulo, Brazil); Jaime Ide (Federal University of São Paulo, Brazil); Alexandre Falcão (Institute of Computing, University of Campinas, Brazil); Chiang-shan Li (Yale University, USA)

pp. 2653-2656

3D Shape Analysis of the Brain Cortex with Application to Dyslexia Matthew J Nitzken (University of Louisville \& Biolmaging Laboratory, USA); Manuel Casanova (University of Louisville, USA); Georgy Gimel'farb (University of Auckland, USA); Ahmed Elnakib (Bioimaging Lab, USA); Fahmi Khalifa (Bioimaging Laboratory \& University of Louisville, Louisville, KY, USA); Andy Switala (University of Louisville, USA); Ayman Sabry El-Baz, PhD (University of Louisville, USA) pp. 2657-2660

Detection of Resting-State Brain Activity in Magnetic Resonance Images Through Wavelet Feature Cluster Analysis

Geert Verdoolaege (Ghent University, Belgium); Leslie Vlerick (Ghent University Hospital, Belgium); Eric Achten (Ghent University Hospital, Belgium) pp. 2661-2664 


\section{WA.PA: Sparse Estimation (Poster)}

Total Variation-Wavelet-Curvelet Regularized Optimization for Image Restoration Shunsuke Ono (Tokyo Institute of Technology, Japan); Takamichi Miyata (Tokyo Institute of Technology, Japan); Katsunori Yamaoka (Tokyo Institute of Technology, Japan) pp. 2665-2668

Image Reconstruction From Compressed Linear Measurements with Side Information Vijayaraghavan Thirumalai (EPFL, Switzerland); Pascal Frossard (Swiss Federal Institute of Technology - EPFL, Switzerland) pp. 2669-2672

Multitemporal Image Change Detection with Compressed Sparse Representation Leyuan Fang (Hunan University, P.R. China); Shutao Li (Hunan University, P.R. China); Jianwen Hu (Hunan University, P.R. China) pp. 2673-2676

Single-View Reconstruction From an Unknown Spherical Mirror Zhihu Chen (The University of Hong Kong, Hong Kong); Kwan-Yee Kenneth Wong (The University of Hong Kong, Hong Kong); Miaomiao Liu (The University of Hongkong, Hong Kong); Dirk Schnieders (The University of Hong Kong, Hong Kong) pp. $2677-2680$

\section{Optical Flow Estimation Using Sparse Gradient Representation} Muhammad Nawaz (University of Wollongong, Australia); Abdesselam Bouzerdoum (University of Wollongong, Australia); Son Lam Phung (University of Wollongong, Australia) pp. 2681-2684

Luminance Constrained Total Variation and Its Application for Optimized Decoding of JPEG 2000

Takamichi Miyata (Tokyo Institute of Technology, Japan); Yoshinori Sakai (Tokyo Institute of Technology, Japan) pp. 2685-2688

Modified-CS-residual for Recursive Reconstruction of Highly Undersampled Functional MRI Sequences Wei Lu (lowa State University, USA); Taoran Li (lowa State University, USA); Ian Atkinson (University of Illinois at Chicago, USA); Namrata Vaswani (lowa State University, USA) pp. 2689-2692

Sparse Representation Based Band Selection for Hyperspectral Images Shuangjiang Li (University of Tennessee at Knoxville, USA); Hairong Qi (the University of Tennessee, USA) pp. 2693-2696

Total-Variation Regularized Motion Estimation in a Periodic Image Sequence Wenyuan Qi (Illinoise Institute of Technology, USA); Xiaofeng Niu (Illinois Institute of Technology, USA); Yongyi Yang (Illinois Institute of Technology, USA) pp. 2697-2700 
3D Image Reconstruction From Sparse Measurement of Wideband Millimeter Wave SAR Experiments

Hamed Kajbaf (Missouri University of Science and Technology, USA); Joseph Case (Missouri University of Science and Technology, USA); Yahong Rosa Zheng (Missouri University of Science and Technology, USA)

pp. 2701-2704

\section{Compressive Passive Millimeter-Wave Imaging}

Sevket Derin Babacan (Northwestern University, USA); Martin Luessi (Northwestern University, USA); Leonidas Spinoulas (Northwestern University, USA); Aggelos K. Katsaggelos (Northwestern University, USA); Nachappa Gopalsami (Argonne National Laboratory, USA); Thomas W Elmer, II (Argonne National Laboratory, USA); Ryan Ahern (Argonne National Laboratory, USA); Shaolin Liao (Argonne National Laboratory, USA); Apostolos Raptis (Argonne National Laboratory, USA) pp. 2705-2708

\section{Hybrid Blind Deconvolution of Images Using Variable Splitting and Proximal Point} Methods

Sudipto Dolui (University of Waterloo, Canada); Oleg Michailovich (University of Waterloo, Canada)

pp. 2709-2712

\section{A New Block Compressive Sensing to Control the Number of Measurements} Hyungkeuk Lee (Yonsei University, Korea); Heeseok Oh (Wireless Network Lab., Yonsei University, Korea); Sanghoon Lee (Yonsei University, Korea) pp. 2713-2716

\section{A Memory Gradient Algorithm for L2-LO Regularization with Applications to Image} Restoration

Emilie Chouzenoux (Université Paris-Est Marne-la-Vallée, France); Jean-Christophe Pesquet (University Paris-Est, France); Hugues Talbot (Université Paris Est, France); Anna Jezierska (Université Paris-Est Marne-la-Vallée, France) pp. $2717-2720$

\section{WA.PB: Data Hiding and Media Security (Poster)}

An Effective Image Steganalysis Method Based on Neighborhood

Qingxiao Guan (University of Science and Technology of China, P.R. China); Jing Dong (Institute of Automation, Chinese Academy of Sciences, P.R. China); Tieniu Tan (NLPR, P.R. China) pp. 2721-2724

Frequency Domain Infrared Watermarking for Printed CMYK Image Yonghui Zhao (Xerox Research Center Webster, USA); Zhigang Fan (Xerox Corporation, USA); Martin Hoover (Xerox Research Center Webster, USA) pp. $2725-2728$

Affine Transformation Invariant Image Watermarking Using Moment Normalization and Radial Symmetry Transform

Athanasios Nikolaidis (Technological Educational Institute of Serres, Greece) pp. $2729-2732$ 


\section{Compression and Protection of JPEG Images}

Yi-Chong Zeng (Academia Sinica, Taiwan); Fay Huang (National Ilan University, Taiwan); Mark Liao (Academia Sinica, Taiwan)

pp. 2733-2736

\section{Secure JPEG Steganography by LSB+ Matching and Multi-Band Embedding} Hao-tian Wu (Sun Yat-Sen University, P.R. China); Jiwu Huang (Sun Yat-sen University, P.R. China) pp. $2737-2740$

\section{Reversible Watermarking Based on Generalized Histogram Shifting} Mohammad Arabzadeh, Mohammad Abadi (Shiraz University of Technology, Iran); Mohammad Sadegh Helfroush (Shiraz University of Technology, Iran); Habibollah Danyali (Shiraz University of Technology, Iran); Keyvan Kasiri (Shiraz University of Technology, Iran) pp. 2741-2744

\section{Robust Watermark Extraction Using SVD-based Dynamic Stochastic Resonance} Rajlaxmi Chouhan (PDPM Indian Institute of Information Technology, Design and Manufacturing Jabalpur, India); Rajib Kumar Jha (PDPM Indian Institute of Information Technology, Design \& Manufacturing Jabalpur, India); Apoorv Chaturvedi (PDPM IIITDM Jabalpur, India); Toshihiko Yamasaki (The University of Tokyo, Japan); Kiyoharu Aizawa (University of Tokyo, Japan) pp. 2745-2748

\section{Improved Multiplicative Spread Spectrum Embedding for Image Data Hiding} Amir Valizadeh (University of British Columbia, Canada); Z. Jane Wang (University of British Columbia, Canada) pp. 2749-2752

\section{Countermeasure of Re-recording Prevention Against Attack with Short Wavelength} Pass Filter

Takayuki Yamada (Graduate University for Advanced Studies, Japan); Gohshi Seiichi (Sharp Corporation, Japan); Isao Echizen (National Institute of Informatics (NII), Japan) pp. $2753-2756$

A New Blind Robust Image Watermarking Scheme in SVD-DCT Composite Domain Zhen Li (NTU, Singapore); Kim Hui Yap (Nanyang Technological University, Singapore); Ying Lei (School of EEE, Nanyang Technological University, Singapore) pp. 2757-2760

Virtual View Invariant Domain for 3D Video Blind Watermarking Javier Franco-Contreras (Technicolor, France); Séverine Baudry (Technicolor, France); Gwenael J Doërr (Technicolor, France) pp. 2761-2764

\section{A Novel Approach to Adaptive Image Authentication} Pawel Korus (AGH University of Science and Technology, Poland); Andrzej Dziech (AGH University of Science and Technology, Poland) pp. 2765-2768 
A Collusion Resilient Key Management Scheme for Multi-dimensional Scalable Media Access Control

Xinglei Zhu (State University of New York at Buffalo, USA); Chang Wen Chen (State University of New York at Buffalo, USA)

pp. 2769-2772

Synchronization of Texture and Depth Map by Data Hiding for 3D H.264 Video

Zafar Shahid (LIRMM, France); William Puech (University of Montpellier \& LIRMM,

France)

pp. 2773-2776

\section{WA.PC: Remote Sensing an Geophysical Imaging (Poster)}

A New Approach to the Automated Mapping of Pockmarks in Multi-Beam Bathymetry Richard Harrison (University of East Anglia \& Gardline Geosurvey, United Kingdom); Valerie Bellec (Norges Geologiske Undersekelse (NGU), Trondheim, Norway); Dave Mann (Gardline Geosurvey, United Kingdom); Wenjia Wang (University of East Anglia, United Kingdom) pp. $2777-2780$

Radio Astronomical Image Deconvolution Using Prolate Spheroidal Wave Functions Sarod Yatawatta (ASTRON, The Netherlands) pp. 2781-2784

Globally Optimal Reconstruction of Millimeter-Wave Radiometric Images with Belief Propagation

Michel Sarkis (Sony Deutschland Gmbh, Germany); Murat Shahrashoub (Sony

Deutschland Gmbh, Germany) pp. 2785-2788

Adaptive Patches for Change Detection

Xing Gong (Insitute of Automation, Chinese Academy of Science \& LIAMA, P.R. China); Thomas Corpetti (CNRS - University Rennes 2, France) pp. 2789-2792

Cascaded Active Learning for Object Retrieval Using Multiscale Coarse to Fine Analysis

Pierre Blanchart (Télécom ParisTech, France); Marin Ferecatu (CNAM, France) pp. 2793-2796

\section{Component-based Restoration of Speckled Images}

Vishal Patel (University of Maryland, USA); Glenn Easley (University of Maryland, USA); Rama Chellappa (University of Maryland, USA)

pp. $2797-2800$

\section{Estimation of an Optimal Spectral Band Combination to Evaluate Skin Disease}

Treatment Efficacy Using Multi-Spectral Images

Sylvain Prigent (INRIA Sophia Antipolis, France); Didier Zugaj (Galderma, France); Xavier Descombes (INRIA, France); Philippe Martel (Galderma, France); Josiane Zerubia (INRIA, Sophia Antipolis, France)

pp. 2801-2804 
Segmenting Extended Structures in Radio Astronomical Images by Filtering Bright Compact Sources and Using Wavelets Decomposition

Marta Peracaula (University of Girona, Spain); Arnau Oliver (University of Girona, Spain); Albert Torrent (University of Girona, Spain); Xavier Lladó (University of Girona, Spain); Jordi Freixenet (University of Girona, USA); Joan Martí (University of Girona, Spain) pp. $2805-2808$

\section{Ground Topography Estimation Over Forests Using PollnSAR Image by Means of} Coherence Set

Bin Zou (Harbin Institute of Technology, P.R. China); Da Lu (Harbin Institute of Technology, P.R. China); Hongjun Cai (Harbin Institute of Technology, P.R. China); Ye Zhang (Harbin Institute of Technology, P.R. China) pp. 2809-2812

\section{A Fast Multiple Birth and Cut Algorithm Using Belief Propagation} Ahmed Gamal-Eldin (INRIA Sophia Antipolis, France); Xavier Descombes (INRIA, France); Guillaume Charpiat (INRIA, Sophia Antipolis, France); Josiane Zerubia (INRIA, Sophia Antipolis, France) pp. 2813-2816

\section{Fast Model of Space-Variant Blurring and Its Application to Deconvolution in Astronomy}

Loïc Denis (Centre de Recherche Astrophysique de Lyon, France); Eric Thiébaut (Centre de Recherche Astrophysique de yon, France); Ferreol Soulez (Universite Lyon 1 \& Centre de Recherche Astronomique de Lyon, France) pp. $2817-2820$

\section{Robust Airplane Detection in Satellite Images} Li Wei (NLPR, Institute of Automation, Chinese Academy of Sciences, P.R. China); Shiming Xiang (Institute of Automation, Chinese Academy of Sciences, P.R. China); Haibo Wang (Institute of Automation, Chinese Academy of Sciences, P.R. China); Chunhong Pan (Institute of Automation, Chinese Academy of Sciences, P.R. China) pp. 2821-2824

\section{Extracting Salient Contour Groups From Cluttered Solar Images Via Markov Random Fields}

Nurcan Durak (University of Louisville, USA); Olfa Nasraoui (University of Louisville, USA)

pp. $2825-2828$

\section{Resolution Assessment in Dynamic Image Formation}

Mark D. Butala (Jet Propulsion Laboratory, USA)

pp. 2829-2832

\section{WA.PD: Image Segmentation (Poster)}

\section{Tensor Vector Field Based Active Contours}

Abhishek Kumar (University of Waterloo, Canada); Alexander Wong (University of Waterloo, Canada); Akshaya Mishra (University of Waterloo, Canada); David Clausi (University of Waterloo, Canada); Paul Fieguth (University of Waterloo, Canada) pp. 2833-2836 
EDLines: Real-Time Line Segment Detection by Edge Drawing (ED)

Cuneyt Akinlar (Anadolu University, Turkey); Cihan Topal (Anadolu University, Turkey) pp. 2837-2840

Incremental Local Hough Transform for Line Segment Extraction

Rui Guerreiro (Institute for Systems and Robotics / Instituto Superior Técnico,

Portugal); Pedro Aguiar (Institute for Systems and Robotics / Instituto Superior

Técnico, Portugal)

pp. 2841-2844

General Adaptive Distance Transforms on Gray Tone Images: Application to Image Segmentation

Jean-Charles Pinoli (Ecole Nationale Supérieure des Mines, France); Johan Debayle (Ecole Nationale Supérieure des Mines, France)

pp. 2845-2848

Image Super-segmentation: Segmentation with Multiple Labels From Shuffled

Observations

Jorge S. Marques (Instituto Superior Técnico \& Instituto de Sistemas e Robotica,

Portugal); Mario A. T. Figueiredo (Instituto Superior Técnico, Portugal)

pp. 2849-2852

Improved Force Field for Vector Field Convolution Method

Andrea Kovacs (Pazmany Peter Catholic University \& Computer and Automation

Research Institute, MTA SZTAKI, Hungary); Tamas Szirányi (Computer and

Automation Research Institute of the Hungarian Academy of Sciences \& Pázmány

Péter Catholic University, Hungary)

pp. 2853-2856

Semi-Automatic 3-D Segmentation of Computed Tomographic Imagery by Iterative

Gradient-Driven Volume Growing

Sreenath Rao Vantaram (Rochester Institute of Technology, USA); Eli Saber

(Rochester Institute of Technology, USA); Sohail A Dianat (Rochester Institute of Technology, USA); Yang Hu (Rochester Institute of Technology, USA); Vishwas Abhyankar (DataPhysics Research Incorporation, USA) pp. $2857-2860$

Robust Segmentation of Relevant Regions in Low Depth of Field Images Franz Graf (Ludwig-Maximilians-Universität München, Germany); Hans-Peter Kriegel (Ludwig-Maximilians-Universität München, Germany); Michael Weiler (LudwigMaximilians-Universität München, Germany) pp. 2861-2864

Multicolor Image Segmentation Using Ambrosio-Tortorelli Approximation Takeshi Asahi (University of Chile, Chile); Jaime Ortega (University of Chile, Chile); Rodrigo Lecaros (University of Chile, Chile) pp. 2865-2868

Supervised Texture Segmentation Through a Multi-Level Pixel-Based Classifier Based on Specifically Designed Filters

Jaime Melendez (Universitat Rovira i Virgili, Spain); Xavier Girones (Universitat Rovira i Virgili, Spain); Domenec Puig (University Rovira i Virgili, Spain)

pp. 2869-2872 
A New Information Fusion Approach for Image Segmentation

Wentao Xu (University of Missouri-Columbia \& East China Normal University, USA);

Ratchadaporn Kanawong (University of Missouri-Columbia, USA); Ye Duan

(University of Missouri, USA); Guixu Zhang (East China Normal University, P.R.

China)

pp. 2873-2876

\section{Robust Free Space Segmentation Using Active Contours and Monocular}

Omnidirectional Vision

Pauline Merveilleux (France, France); Ouiddad Labbani-Igbida (MIS, France); EI

Mustapha Mouaddib (MIS, France)

pp. $2877-2880$

Higher Order Potentials with Superpixel Neighbourhood (Hsn) for Semantic Image

\section{Segmentation}

Mostafa S Ibrahim (Microsoft, Egypt); Motaz El-Saban (Microsoft Research - Cairo

Innovation Lab, Egypt)

pp. 2881-2884

An Intensity-Gradient-Texture Guided Methodology for Spatial Segmentation of Remotely Sensed Multi/Hyperspectral Imagery

Sreenath Rao Vantaram (Rochester Institute of Technology, USA); Eli Saber (Rochester Institute of Technology, USA); David Messinger (Rochester Institute of Technology, USA)

pp. 2885-2888

\section{WA.PE: Image Analysis (Poster)}

Nonparametric Polygonal and Multimodel Approximation of Digital Curves with RateDistortion Curve Modeling

Alexander Kolesnikov (University of Eastern Finland, Finland)

pp. 2889-2892

Concentric Ring Signature Descriptor for 3D Objects

Hien Van Nguyen (University of Maryland, USA); Fatih Porikli (Mitsubishi Electric

Research Laboratories, USA)

pp. 2893-2896

Edgelet Tracking Using Gauss-Laguerre Circular Harmonic Filters

Lorenzo Sorgi (Via Maiorise \& CIRA, Italy)

pp. 2897-2900

Graph-based Shape Matching for Deformable Objects

Hanbyul Joo (Electronics and Telecommunications Research Institute, Korea);

Yekeun Jeong (KAIST, Korea); Olivier Duchenne (Ecole Normale Superieure,

France); In-So Kweon (Korea Advanced Institute of Science and Technology (KAIST), Korea)

pp. 2901-2904

Change-detection Based on Support Vector Data Description Handling Dependency Akram Belghith (University of Strasbourg, France); Christophe Collet (Louis Pasteur University, France); Jean Paul Armspach (University of Strasbourg, France) 
pp. $2905-2908$

BOSSA: Extended BoW Formalism for Image Classification

Sandra Avila (Federal University of Minas Gerais \& Universite Pierre et Marie Curie, Brazil); Nicolas Thome (University Pierre et Marie Curie, France); Matthieu Cord (UPMC Paris 6, France); Eduardo Valle (State University of Campinas \& RECOD Lab, Brazil); Arnaldo Araújo (Federal University of Minas Gerais, Brazil) pp. 2909-2912

\section{Object Color Categorization in Surveillance Videos}

Yimeng Zhang (Cornell University, USA); Cheng-Chuan Chou (Industrial Technology Research Institute, Taiwan); Shiaw-Shian Yu (Industrial Technology Research Institute, Taiwan); Tsuhan Chen (Cornell University, USA) pp. 2913-2916

Reconstructing the Drawing Process of Reproductions From Medieval Images Antonio Monroy (IWR - University of Heidelberg, Germany); Bernd Carqué (University of Heidelberg, Germany); Björn Ommer (IWR - University of Heidelberg, Germany) pp. 2917-2920

Preliminary Study on Statistical Shape Model Applied to Diagnosis of Liver Cirrhosis Shinya Kohara (Ritsumeikan University, Japan) pp. 2921-2924

Fast Approximation for Geometric Classification of LiDAR Returns Xiaozhe Shi (University of California, Berkeley, USA); Avideh Zakhor (University of California at Berkeley, USA) pp. 2925-2928

Extraction of Road Network Using a Modified Active Contour Approach Said Mssedi (EPT, Tunisia); Mohamed Ben Salah (INRS, Canada); Riadh Abdelfattah (Ecole Supérieure des Communications, Tunisia); Amar Mitiche (Institut National de la Recherche Scientifique (INRS), Canada) pp. 2929-2932

Uniqueness for Shape From Shading Via Photometric Stereo Technique Roberto Mecca (Sapienza - University of Rome, Italy) pp. 2933-2936

\section{WA.PF: Video Surveillance and Video Conferencing (Poster)}

\section{Real-time Clothing Recognition in Surveillance Videos}

Ming Yang (NEC Laboratories America, USA); Kai Yu (NEC Laboratories America, USA)

pp. $2937-2940$

\section{Real-Time Traffic Analysis At Night-Time}

Jose M. Mossi (Polytechnic University of Valencia, Spain); Alberto Albiol (Universidad Politecnica de Valencia, Spain); Antonio Albiol (Universidad Politecnica Valencia, Spain); Valery Naranjo Ornedo (Polytechnic University of Valencia, Spain) pp. 2941-2944 
A Video Analytics Framework for Amorphous and Unstructured Anomaly Detection Martin Mueller (Georgia Institute of Technology, USA); Peter Karasev (Georgia Institute of Technology, USA); Ivan Kolesov (Georgia Institute of Technology, USA); Allen Tannenbaum (Georgia Institute of Technology, USA) pp. $2945-2948$

PTZ Camera-Based Adaptive Panoramic and Multi-layered Background Model Kang Xue (Beijing Institute of Technology \& Georgia Institute of Technology, P.R. China) pp. 2949-2952

\section{Background Subtraction Through Multiple Life Span Modeling} Junliang Xing (Tsinghua University, P.R. China); Liwei Liu (Tsinghua University, P.R. China); Haizhou Ai (Tsinghua University, P.R. China) pp. 2953-2956

\section{Common Visual Pattern Discovery Via Directed Graph Model}

Chen Wang (Nanyang Technological University \& Temasek Lab @ NTU, Singapore); Kai-Kuang Ma (Nanyang Technological University, Singapore) pp. 2957-2960

\section{Visual Framing Feedback for Desktop Video Conferencing} Chen Wu (Google Inc., USA); Ramin Samadani (HP Labs, USA); April Slayden Mitchell (Hewlett-Packard, USA); Mary G. Baker (HP Labs, USA); Dan Gelb (HewlettPackard Labs, USA) pp. 2961-2964

An Unorthodox Approach Towards Shape From Focus Mannan Muhammad (Gwangju Institute of Science and Technology, Korea); Tae-Sun Choi (Gwangju Institute of Science and Technology, Korea) pp. 2965-2968

\section{A Novel Framework for Automatic Passenger Counting}

Satarupa Mukherjee (University of Alberta, Canada); Baidya Nath Saha (University of Alberta, Canada); Iqbal Jamal (AQL Management Consulting Inc., Canada); Richard Leclerc (City of Edmonton, Canada); Nilanjan Ray (University of Alberta, Canada) pp. 2969-2972

Fire Scene Segmentations for Forest Fire Characterization: a Comparative Study Jean-François Collumeau (University of Orléans, France); Helene Laurent (ENSI de Bourges \& Institut PRISME, France); Adel Hafiane (ENSI de Bourges - Institut PRISME, France); Khaled Chetehouna (ENSI de Bourges, France) pp. 2973-2976

Semi-supervised Learning with Kernel Locality-constrained Linear Coding Yao-Jen Chang (Cornell University, USA); Tsuhan Chen (Cornell University, USA) pp. 2977-2980

\section{Softferns for Homography Estimation}

ShaoGuo Liu (Institute of Automation, Chinese Academy of Sciences, P.R. China); Haibo Wang (Institute of Automation, Chinese Academy of Sciences, P.R. China); Jixia Zhang (Institute of Automation, Chinese Academy of Sciences, P.R. China); Franck Davoine (CNRS, P.R. China); Chunhong Pan (Institute of Automation, Chinese Academy of Sciences, P.R. China) pp. 2981-2984 


\section{Automatic Image Orientation Detection with Prior Hierarchical Content-Based Classification \\ Ivana Cingovska (Skopje, Macedonia); Zoran Ivanovski (Ss. Cyril and Methodius University, Macedonia); François Martin (NXP Software B. V. Eindhoven, France) pp. 2985-2988}

\section{Blackboard Content Classification for Lecture Videos}

Ali Shariq Imran (Gjøvik University College, Norway); Faouzi Alaya Cheikh (Gjovik University College, Norway)

pp. 2989-2992

\section{Real-time Affine Invariant Patch Matching Using DCT Descriptor and Affine Space Quantization}

Xiaobo Chen (Beijing University of Posts and Telcommunications, P.R. China); Ye Feng (Beijing university of Posts and telecommunications, P.R. China); Men Aidong (Beijing University of Posts and Telecommunication, P.R. China) pp. 2993-2996

\section{WA.PG: Image Processing Methods for Face Recognition (Poster)}

\section{Local Color Vector Binary Pattern for Face Recognition}

Seung-Ho Lee (Korea Advanced Institute of Science and Technology (KAIST), Korea); JaeYoung Choi (Korea Advanced Institute of Science and Technology (KAIST), Korea); Konstantinos N Plataniotis (University of Toronto, Canada); Yong Man Ro (KAIST, Korea)

pp. 2997-3000

\section{Facial Expression Recognition Using Clustering Discriminant Non-negative Matrix Factorization}

Symeon Nikitidis (Aristotle University of Thessaloniki \& CERTH ITI, Greece); Anastasios Tefas (Aristotle University of Thessaloniki, Greece); Nikos Nikolaidis (Aristotle University of Thessaloniki \& Informatics and Telematics Institute, CERTH, Greece); Ioannis Pitas (Aristotle University of Thessaloniki, Greece) pp. 3001-3004

A Novel Kernel Discriminant Feature Extraction Framework Based on Mapped Virtual Samples for Face Recognition

Sheng Li (Nanjing University of Posts and Telecommunications, P.R. China); Xiaoyuan Jing (College of Automation, Nanjing University of Posts and Telecommunications \& State Key Laboratory for Software Engineering, Wuhan University, P.R. China); David Zhang (The Hong Kong Polytechnic University, Hong Kong); Yongfang Yao (Nanjing University of Posts and Telecommunications, P.R. China); Lusha Bian (Nanjing University of Posts \& Telecommunications, P.R. China) pp. 3005-3008

\section{Kernel Sparse Representation with Local Patterns for Face Recognition} Cuicui Kang (National Laboratory of Pattern Recognition, P.R. China); Shengcai Liao (Institute of Automation, Chinese Academy of Sciences, P.R. China); Shiming Xiang (Institute of Automation, Chinese Academy of Sciences, P.R. China); Chunhong Pan (Institute of Automation, Chinese Academy of Sciences, P.R. China) pp. 3009-3012 
Discriminant Subclass-center Manifold Preserving Projection for Face Feature Extraction

Chao Lan (Nanjing University of Posts and Telecommunications, P.R. China);

Xiaoyuan Jing (College of Automation, Nanjing University of Posts and Telecommunications \& State Key Laboratory for Software Engineering, Wuhan University, P.R. China); David Zhang (The Hong Kong Polytechnic University, Hong Kong); Shiqiang Gao (Nanjing University of Posts and Telecommunications, P.R. China); Jingyu Yang (Nanjing University of Science and Technology, P.R. China) pp. 3013-3016

Texture Classification Based Low Order Local Binary Pattern for Face Recognition Ching-Te Chiu (National Tsing Hua University, Taiwan); Cyuan Jhe Wu (National Tsing Hua University, Taiwan) pp. 3017-3020

Manifold Learning for Simultaneous Pose and Facial Expression Recognition Raymond Ptucha (Rochester Institute of Technology, USA); Grigorios Tsagkatakis (Rochester Institute of Technology, USA); Andreas Savakis (Rochester Institute of Technology, USA) pp. 3021-3024

Multi-view Face Recognition Via Joint Dynamic Sparse Representation Haichao Zhang (Northwestern Polytechnical University \& University of Illinois at Urbana-Champaign, USA); Nasser Nasrabadi (US Army Research Laboratory, USA); Thomas S Huang (University of Illinois at Urbana-Champaign, USA); Yanning Zhang (Northwestern Polytechnical University, P.R. China) pp. 3025-3028

Local Primitive Code Mining for Fast and Accurate Face Recognition Li Jiangwei (Nokia Research Center, P.R. China); Lei Xu (Nokia Research Center, P.R. China); Kongqiao Wang (Nokia Research Center, P.R. China); Ma Yong (Nokia Research Center, P.R. China); Xiong Tao (Nokia Research Center, P.R. China) pp. 3029-3032

Robust Low-Rank Subspace Recovery and Face Image Denoising for Face Recognition

Mingyang Jiang (Peking University, P.R. China); Jufu Feng (Peking University, P.R. China)

pp. 3033-3036

\section{A Mixture of Gated Experts Optimized Using Simulated Annealing for 3D Face Recognition}

Wael Ben Soltana (LIRIS ECL, France); Di Huang (Ecole Centrale Lyon, France); Mohsen Ardabilian (Ecole Centrale Lyon \& LIRIS LAB-UMR 5205 CNRS, France); Liming Chen (EC Lyon, France); Chokri Ben Amar (University of Sfax, National School of Engineers, Tunisia) pp. 3037-3040

Semi-supervised Face Recognition with LDA Self-training Xuran Zhao (EURECOM, France); Nicholas Evans (EURECOM, France); Jean-Luc Dugelay (Institut EURECOM, France) pp. 3041-3044 
Robust Facial Expression Tracking Based on Composite Constraints AAM Xuetao Feng (Samsung Advanced Institute of Technology, P.R. China); Xiaolu Shen (Samsung Advanced Institute of Technology, P.R. China); Mingcai Zhou (Samsung Advanced Institute of Technology, P.R. China); Hui Zhang (Samsung Advanced Institute of Technology, P.R. China); Jungbae Kim (Samsung Advanced Institute of Technology, Korea)

pp. 3045-3048

Face Recognition Based on Local Uncorrelated and Weighted Global Uncorrelated Discriminant Transforms

Xiaoyuan Jing (College of Automation, Nanjing University of Posts and Telecommunications \& State Key Laboratory for Software Engineering, Wuhan University, P.R. China); Sheng Li (Nanjing University of Posts and Telecommunications, P.R. China); David Zhang (The Hong Kong Polytechnic University, Hong Kong); Jingyu Yang (Nanjing University of Science and Technology, P.R. China) pp. 3049-3052

\section{Expression Robust 3D Face Recognition Via Mesh-based Histograms of Multiple} Order Surface Differential Quantities

Huibin Li (Ecole Centrale de Lyon, France); Di Huang (Ecole Centrale Lyon, France); Pierre Lemaire (Ecole Centrale de Lyon, France); Jean-Marie Morvan (Universite Claude Bernard, France); Liming Chen (EC Lyon, France) pp. 3053-3056

\section{WP.I: Round Table on Reproducible Research}

\section{WP.L1: Analysis of Microscopy and Reconstructive Images for Applications in Medicine and Biology (Special Session)}

3D Microscopic Imaging by Synchrotron Radiation micro/nano-CT

Francoise Peyrin (Universite de Lyon INSA Lyon \& Inserm U1044 UMR CNRS 5220, France); Alexandra Pacureanu (Université de Lyon, France); Max Langer (Université de Lyon, France)

pp. $3057-3060$

The Formulation of a Non-Linear Hertzian Model in Order to Assess the Mechanical Strength of Human Cells Based on Data From an Atomic Force Microscope

David Burton (Liverpool John Moores University, United Kingdom); Mark Murphy (Liverpool John Moore University, United Kingdom); Francis Lilley (Liverpool John Moores University, United Kingdom); Munther A Gdeisat (Liverpool John Moores University, United Kingdom) pp. 3061-3064

A Novel Technique for the Restoration of Atomic Force Microscope Images Enabling an Approximation of AFM Impulse Response

Ahmed Ahtaiba (Liverpool John Moores University, United Kingdom); Munther A Gdeisat (Liverpool John Moores University, United Kingdom); David Burton (Liverpool John Moores University, United Kingdom); Francis Lilley (Liverpool John Moores 
University, United Kingdom); Mark Murphy (Liverpool John Moore University, United Kingdom); Gary Johnston (Liiverpool John Moores University, United Kingdom) pp. 3065-3068

Analysis of Microscopy and Reconstructive Images for Applications in Medicine and Biology

Gary Johnston (Liiverpool John Moores University, United Kingdom); David Burton (Liverpool John Moores University, United Kingdom); Francis Lilley (Liverpool John Moores University, United Kingdom); Annette Doyle (Liverpool John Moores University, United Kingdom); Mark Murphy (Liverpool John Moore University, United Kingdom); Greg Madden (Liverpool John Moores University, United Kingdom); Munther A Gdeisat (Liverpool John Moores University, United Kingdom); Christopher Moore (The Christie NHS Foundation Trust, United Kingdom); Tom Marchant (The Christie NHS Foundation Trust, United Kingdom); Bogdan J Matuszewski (University of Central Lancashire, United Kingdom)

pp. 3069-3072

break

\author{
Numerical Evaluation of Sampling Bounds for Near-Optimal Reconstruction in \\ Compressed Sensing \\ Yoann Le Montagner (Institut Pasteur, France); Marcio Marim (Institut Pasteur, \\ France); Elsa Angelini (Télécom ParisTech, France); Jean-Christophe Olivo-Marin \\ (Institut Pasteur, France) \\ pp. 3073-3076
}

\title{
Confocal Microscopy Segmentation Using Active Contour Based on Alpha- Divergence
}

Leila Meziou (ETIS UMR CNRS 8051, France); Aymeric Histace (ETIS UMR CNRS 8051 \& University of Cergy-Pontoise, ENSEA, France); Frederic Precioso (LIP6 CNRS UMR 7606 \& UPMC Paris 6, France); Bogdan J Matuszewski (University of Central Lancashire, United Kingdom); Mark Murphy (Liverpool John Moore University, United Kingdom) pp. 3077-3080

\section{Segmentation of Cellular Structures in Actin Tagged Fluorescence Confocal Microscopy Images}

Bogdan J Matuszewski (University of Central Lancashire, United Kingdom); Mark Murphy (Liverpool John Moore University, United Kingdom); David Burton (Liverpool John Moores University, United Kingdom); Tom Marchant (The Christie NHS Foundation Trust, United Kingdom); Christopher Moore (The Christie NHS Foundation Trust, United Kingdom); Aymeric Histace (ETIS UMR CNRS 8051 \& University of Cergy-Pontoise, ENSEA, France); Frederic Precioso (LIP6 CNRS UMR 7606 \& UPMC Paris 6, France) pp. 3081-3084

Quantifying Structure Regularity in Fluorescence Microscopy Cell Images Using a Novel Multi-Dimensional Approximate Entropy Metric

Tom Marchant (The Christie NHS Foundation Trust, United Kingdom); Mark Murphy (Liverpool John Moore University, United Kingdom); Greg Madden (Liverpool John Moores University, United Kingdom); Christopher Moore (The Christie NHS Foundation Trust, United Kingdom) pp. 3085-3088 


\section{WP.L2: Image Quality Assessment (Lecture)}

\section{No-reference Image Quality Assessment Based on Visual Codebook}

Peng Ye (University of Maryland, College Park, USA); David Doermann (University of Maryland Institute for Advanced Computer Studies, USA)

pp. 3089-3092

\section{DCT Statistics Model-Based Blind Image Quality Assessment}

Michele Saad (The University of Texas at Austin, USA); Alan C Bovik (University of Texas at Austin, USA); Christophe Charrier (Universite de Caen Basse-Normandie, France)

pp. 3093-3096

\section{Crowdsourcing Subjective Image Quality Evaluation}

Flavio Ribeiro (University of São Paulo, Brazil); Dinei Florencio (Microsoft Research, USA); Vitor H Nascimento (USP, Brazil) pp. 3097-3100

\section{Systematic Stress Testing of Image Quality Estimators}

Frank Ciaramello (Cornell University, USA); Amy Reibman (AT\&T Labs - Research, USA)

pp. 3101-3104

break

\section{Objective Metrics for Quality of Experience in Stereoscopic Images}

Liyuan Xing (Q2S-NTNU, Norway); Junyong You (Norwegian University of Science and Technology, Norway); Touradj Ebrahimi (EPFL, Switzerland); Andrew Perkis (NTNU, Norway)

pp. 3105-3108

Assessing the Quality of Compressed Images Using EEG

Lea Lindemann (TU Braunschweig, Germany); Marcus Magnor (TU Braunschweig, Germany)

pp. 3109-3112

Image Quality Assessment of Endoscopic Panorama Images

Alexander Behrens (RWTH Aachen University, Germany); Michael Bommes (RWTH Aachen University, Germany); Sebastian Gross (RWTH Aachen University,

Germany); Til Aach (RWTH Aachen University, Germany) pp. 3113-3116

\section{Comprehensive Assessment of Iris Image Quality}

Xingguang Li (University of Science and Technology of China, P.R. China); Zhenan Sun (Achinese Academy of Sciences, P.R. China); Tieniu Tan (NLPR, P.R. China) pp. 3117-3120 


\section{WP.L3: Video and Multichannel Segmentation (Lecture)}

Towards Real-Time 3D Region-Based Segmentation: B-Spline Explicit Active Surfaces Daniel Barbosa (Katholieke Universiteit Leuven, Portugal); Jan D'hooge (Cardiac Imageing Research, Belgium); Thomas Dietenbeck (CREATIS, France); Denis Friboulet (CREATIS, France); Olivier Bernard (Creatis, France) pp. 3121-3124

\section{Segmentation by Temporal Detection Integration}

Yi-Ying Wang (Hermes Microvision Inc, Taiwan); Chia-han Lee (Academia Sinica, Taiwan)

pp. $3125-3128$

\section{Image Labeling by Multiple Segmentation}

Quan Zhou (Department of Electronics and Information Engineering, Huazhong University of Science and Technology, P.R. China); Canxiang Yan (Huazhong University of Science and Technology, P.R. China); Yingying Zhu (Huazhong University of Science and Technology, P.R. China); Xiang Bai (Huazhong University of Science and Technology, P.R. China); Wenyu Liu (Huazhong University of Science and Technology, P.R. China)

pp. 3129-3132

The Riverbed Approach for User-steered Image Segmentation

Paulo Miranda (University of Campinas \& Institute of Computing, Brazil); Alexandre Falcão (Institute of Computing, University of Campinas, Brazil); Thiago Spina (University of Campinas, Brazil)

pp. 3133-3136

break

Clothing Segmentation and Recoloring Using Background Subtraction and Back Projection Method

Susu Yao (Institute for Infocomm Research, Singapore); Ishtiaq Rasool Khan (A*STAR Institute for Infocomm Research, Singapore); Farzam Farbiz (A-Star Institute for Infocomm Research, Singapore)

pp. 3137-3140

\section{Harmonic Active Contours for Multichannel Image Segmentation}

Virginia Estellers (Ecole Polytechnique Federale de Lausanne (EPFL), Switzerland); Dominique Zosso (Ecole Polytechnique Fédérale de Lausanne (EPFL), Switzerland); Xavier Bresson (City University of Hong Kong, Hong Kong); Jean-Philippe Thiran (École Polytechnique Fédérale de Lausanne \& Signal Processing Laboratory, Switzerland) pp. 3141-3144

\section{Automatic Fish Segmentation Via Double Local Thresholding for Trawl-Based} Underwater Camera Systems

Meng-Che Chuang (University of Washington, USA); Jenq-Neng Hwang (University of Washington, USA); Kresimir Williams (National Oceanic and Atmospheric Administration, USA); Richard Towler (National Oceanic and Atmospheric Administration, USA) pp. 3145-3148 
Automatic People Segmentation with a Template-Driven Graph Cut

Cyrille Migniot (Grenoble Institute of Technology \& GIPSA-LAB, France); Pascal Bertolino (Grenoble Institute of Technology, France); Jean-Marc Chassery (Grenoble Institute of Technology \& GIPSA-LAB, France)

pp. 3149-3152

\section{WP.L4: Color/Multispectral Imaging and Demosaicking (Lecture)}

New Color Filter Arrays of High Light Sensitivity and High Demosaicking Performance Jue Wang (Peking Univiersity, P.R. China); Chao Zhang (Peking University, P.R. China); Pengwei Hao (Queen Mary, University of London, United Kingdom) pp. 3153-3156

Multispectral Demosaicking Using Adaptive Kernel Upsampling

Yusuke Monno (Tokyo Institute of Technology, Japan); Masayuki Tanaka (Tokyo Institute of Technology, Japan); Masatoshi Okutomi (Tokyo Institute of Technology, Japan)

pp. 3157-3160

Disregarding Spectral Overlap - a Unified Approach for Demosaicking, Compressive Sensing and Color Filter Array Design

Tripurari Singh (Image Algorithmics, USA); Mritunjay Singh (Image Algorithmics, USA)

pp. 3161-3164

Correlation-based Joint Acquisition and Demosaicing of Visible and Near-Infrared Images

Zahra Sadeghipoor (EPFL, Switzerland); Yue M. Lu (Harvard University, USA);

Sabine Süsstrunk (EPFL, Switzerland)

pp. 3165-3168

break

Increasing Camera Dynamic Range Through In-Sensor Multi-Exposure White Balancing

Michael Schöberl (University of Erlangen-Nuremberg, Germany); Wolfgang Schnurrer (University of Erlangen Nuremberg, Germany); Siegfried Foessel (Fraunhofer IIS, Germany); Andre Kaup (University of Erlangen-Nuremberg, Germany) pp. 3169-3172

\section{Multi-dimensional Earth Mover's Distance Active Contours}

Carlos S. Mendoza (University of Sevilla, Spain); Germán Bohórquez-Ruiz (University of Sevilla, Spain); Begoña Acha (University of Sevilla, Spain); Carmen Serrano (University of Sevilla, Spain)

pp. 3173-3176

\section{Good Looking Green Images}

Hadi Hadizadeh (Simon Fraser University, Canada); Ivan V. Bajic (Simon Fraser University, Canada); Parvaneh Saeedi (Simon Fraser University, Canada); Scott Daly (Dolby Laboratories, USA)

pp. 3177-3180 
Edge Detection in Multispectral Images Using the N-Dimensional Self-Organizing Map Johannes Jordan (University of Erlangen-Nuremberg, Germany); Elli Angelopoulou (Friedrich-Alexander University Erlangen-Nuremberg, Germany) pp. 3181-3184

\section{WP.L6: Hand and Iris-based Biometrics (Lecture)}

\section{Contact-Free Hand Geometry Identification System}

Jing-Ming Guo (National Taiwan University of Science and Technology, Taiwan); YunFu Liu (National Taiwan University of Science and Technology, Taiwan)

pp. 3185-3188

Deformable DAISY Matcher for Robust Iris Recognition Man Zhang (Chinese Academy of Sciences, Institute of Automation, P.R. China); Zhenan Sun (Achinese Academy of Sciences, P.R. China); Tieniu Tan (NLPR, P.R. China) pp. 3189-3192

Incorporating Color Information for Reliable Palmprint Authentication

Aythami Morales (Universidad de Las Palmas de Gran Canaria \& University Las Palmas de Gran Canaria, Spain); Ajay Kumar (The Hong Kong Polytechnic University, Hong Kong); Miguel A. Ferrer (Las Palmas de Gran Canaria University, Spain) pp. 3193-3196

\section{Feature-domain Super-Resolution for Iris Recognition}

Kien Nguyen (Queensland University of Technology, Australia); Clinton Fookes (Queensland University of Technology, Australia); Sridha Sridharan (Queensland University of Technology, Australia); Simon Denman (Queensland University of Technology, Australia)

pp. 3197-3200

break

\section{A Novel Fingerprint Matching Algorithm Using Minutiae Phase Difference Feature} Chongjin Liu (Peking University, Beijing, P.R. China); Jia Cao (Peking University, Beijing, P.R. China); Xin Gao (Peking University, Beijing, P.R. China); Xiang Fu (Peking University, Beijing, P.R. China); Jufu Feng (Peking University, P.R. China) pp. 3201-3204

Fast and Accurate Iris Segmentation Based on Linear Basis Function and RANSAC Kai Wang (Zhejiang University, P.R. China); Yuntao Qian (Zhejiang University, P.R. China) pp. 3205-3208

Front View Gait Recognition Using Spherical Space Model with Human Point Clouds Jegoon Ryu (Waseda University, Japan); Sei-ichiro Kamata (Waseda University, Japan) pp. 3209-3212 
Security Analysis of a Cancelable Iris Recognition System Based on Block

Remapping

Stefan Jenisch (University of Salzburg, Austria); Andreas Uhl (Salzburg University, Austria)

pp. 3213-3216

\section{WP.L7: Video Streaming and Error-Resilient Coding (Lecture)}

Fast Mode Decision for H.264 Video Coding in Packet Loss Environment

Yuan Zhang (Communication University of China \& University of California, San

Diego, USA); Pamela Cosman (University of California, San Diego, USA) pp. $3217-3220$

\section{A Unified Framework for Spectral Domain Prediction and End-To-End Distortion Estimation in Scalable Video Coding}

Jingning Han (University of California Santa Barbara, USA); Vinay Melkote (Dolby Laboratories Inc., USA); Kenneth Rose (University of California, Santa Barbara, USA) pp. 3221-3224

Face Recovery in Conference Video Streaming Using Robust Principal Component Analysis

Wai-tian Tan (Hewlett-Packard, USA); Gene Cheung (National Institute of Informatics, Japan); Yi Ma (University of Illinois at Urbana-Champaign, USA) pp. 3225-3228

Enhanced Error Resiliency for Video with Cyclic Intra-Refresh Lines

Sandro Moiron (Instituto de Telecomunicações \& University of Essex, Portugal); Mohammad Ghanbari (University of Essex, United Kingdom) pp. 3229-3232

break

Prioritized Packet Fragmentation for H.264 Video

Kashyap Kambhatla (University of California San Diego and San Diego State University, USA); Sunil Kumar (San Diego State University, USA); Pamela Cosman (University of California, San Diego, USA) pp. 3233-3236

Rate-distortion-optimized Content-adaptive Coding for Immersive Networked Experience of Sports Events Haopeng Li (Royal Institute of Technology, Sweden); Markus Flierl (KTH Royal Institute of Technology, Sweden) pp. $3237-3240$

A Robust Content-Based JPWL Transmission Over a Realistic MIMO Channel Under Perceptual Constraints

Julien Abot (University of Poitiers, France); Michael Nauge (XLIM-SIC CNRS, France); Clency Perrine (Université de Poitiers, France); Chaker Larabi (Université de Poitiers \& XLIM-SIC, France); Cyril Bergeron (Thalès Communictaions, France); Yannis Pousset (XLIM-SIC CNRS, France); Christian Olivier (XLIM-SIC CNRS, France) pp. 3241-3244 
Cross-Layer Design for Video Streaming with Dynamic Antenna Selection Ching-Hui Chen (Academia Sinica, Taiwan); Wei-Ho Chung (Academia Sinica, Taiwan); Yu-Chiang Frank Wang (Academia Sinica, Taiwan) pp. $3245-3248$

\section{WP.L8: Human Behavior Analysis and Foreground/Background Separation (Lecture)}

\section{A Dataset for Workflow Recognition in Industrial Scenes}

Athanasios Voulodimos (National Technical University of Athens, Greece); Dimitris Kosmopoulos (NCSR Demokritos, Greece); Georgios Vasileiou (NCSR Demokritos, Greece); Emmanuel S. Sardis (National Technical University of Athens - NTUA, Greece); Anastasios D. Doulamis (National Technical University of Athens, Greece); Vassilios Anagnostopoulos (National Technical University of Athens, Greece); Constantinos G Lalos (National technical University of Athens, Greece); Theodora Varvarigou (National Technical University of Athens, Greece) pp. 3249-3252

Active Learning for Human Action Recognition with Gaussian Processes Xianghang Liu (University of New South Wales \& National ICT Australia, Australia); Jian Zhang (The University of New South Wales \& NICTA, Australia) pp. 3253-3256

System for the Automated Segmentation of Heads From Arbitrary Background Benjamin Prestele (Fraunhofer HHI, Germany); David Schneider (Fraunhofer HHI, Germany); Peter Eisert (Fraunhofer HHI \& Humboldt University, Germany) pp. $3257-3260$

Robust Density Modelling Using the Student's T-Distribution for Human Action Recognition

Zia Moghaddam (University of Technology, Sydney, Australia); Massimo Piccardi (University of Technology, Sydney, Australia) pp. 3261-3264

break

Incorporating Estimated Motion in Real-time Background Subtraction Minglun Gong (Memorial University of Newfoundland, Canada); Li Cheng (NICTA and Australian National University, Australia) pp. 3265-3268

Foreground Estimation Based on Robust Linear Regression Model Gengjian Xue (Shanghai Jiao Tong University, P.R. China); Li Song (Shanghai Jiao Tong University, P.R. China); Jun Sun (Shanghai Jiao Tong University, P.R. China); Meng Wu (Shanghai Jiao Tong University, P.R. China) pp. 3269-3272

\section{Selective Subtraction When the Scene Cannot Be Learned} Adeel Bhutta (University of Central Florida, USA); Imran Junejo (University of Sharjah, UAE); Hassan Foroosh (University of Central Florida, USA) pp. 3273-3276 
Selective Eigenbackgrounds Method for Background Subtraction in Crowed Scenes Zhipeng Hu (Institute of Computing Technology, Chinese Academy of Sciences \& National Engineering Laboratory for Video Technology, Peking University, P.R. China); Yaowei Wang (Beijing Institute of Technology, P.R. China); Yonghong Tian (National Engineering Lab for Video Technology, Peking University, P.R. China); Tiejun Huang (Peking University, P.R. China) pp. 3277-3280

\section{WP.PA: Multimedia Quality Assessment and Modeling of Visual Perception (Poster)}

Image Complexity Measure Based on Visual Attention

Matthieu Perreira Da Silva (University of La Rochelle, France); Vincent Courboulay

(University of La Rochelle, France); Pascal Estraillier (University of La Rochelle,

France)

pp. 3281-3284

Robustness and Repeatability of Saliency Models Subjected to Visual Degradations Olivier Le Meur (University of Rennes 1, France)

pp. 3285-3288

\section{Visual Attention: Effects of Blur}

Rizwan Ahmed Khan (LIRIS, Université Claude Bernard Lyon 1 \& Laboratoire Hubert Curien, Saint-Etienne, France); Eric Dinet (Laboratoire Hubert Curien, France);

Hubert Konik (Laboratoire Hubert Curien, France)

pp. 3289-3292

\section{Tracking Failure Detection by Imitating Human Visual Perception}

Hyung Jin Chang (Seoul National University \& Perception and Intelligence Laboratory, Korea); Myoung Soo Park (Seoul National University, Korea); Hawook Jeong (Seoul National University \& Perception and Intelligence Laboratory, Korea); Jin Young Choi (Seoul National University, Korea) pp. 3293-3296

\section{Visual Attention Based Image Quality Assessment}

Anan Guo (Harbin Institute of Technology, P.R. China); Debin Zhao (Harbin Institute of Technology, P.R. China); Shaohui Liu (Harbin Institute of Technology, P.R. China); Xiaopeng Fan (Hong Kong University of Science and Technology, P.R. China); Wen Gao (ICT-ISVISION Joint R\&D Laboratory for Face Recognition, CAS, P.R. China) pp. $3297-3300$

\section{Fusion of Visual Attention Cues by Machine Learning}

Wen-Fu Lee (National Taiwan University, Taiwan); Tai-Hsiang Huang (National Taiwan University, Taiwan); Su-Ling Yeh (National Taiwan University, Taiwan); Homer Chen (National Taiwan University, Taiwan) pp. 3301-3304

\section{Edge-Based Objective Evaluation of Image Quality}

Boban Bondzulic (Military Academy, Serbia); Vladimir Petrovic (University of Manchester, United Kingdom)

pp. 3305-3308 
An Image Quality Assessment Metric Based on Non-Shift Edge

Wufeng Xue (Xi'an Jiaotong University, P.R. China); Xuanqin Mou (Xi'an Jiaotong University, P.R. China)

pp. 3309-3312

RRAR: A Novel Reduced-Reference IQA Algorithm for Facial Images

Jiazhen Zhu (Shanghai University, P.R. China); Yuchun Fang (Shanghai University, P.R. China); Pengjun Ji (Shanghai University, P.R. China); Moad Abdl (Shanghai University, P.R. China); Wang Dai (Shanghai University, P.R. China) pp. 3313-3316

\section{Calibrating MS-SSIM for Compression Distortions Using MLDS}

Christophe Charrier (Universite de Caen Basse-Normandie, France); Kenneth Knoblauch (INSERM U846, France); Laurence Maloney (Univerity of New-York, USA); Alan C Bovik (University of Texas at Austin, USA) pp. $3317-3320$

Modeling of Rate and Perceptual Quality of Video and Its Application to Frame Rate Adaptive Rate Control

Zhan Ma (Samsung Telecommunications America, USA); Meng Xu (Polytechnic Institute of New York University, USA); Kyeong Yang (Dialogic Inc, USA); Yao Wang (Polytechnic Institute of NYU, USA) pp. 3321-3324

No-Reference Video Quality Metric for HDTV Based on H.264/AVC Bitstream Features Christian Keimel (Technische Universität München, Germany); Manuel Klimpke (Technische Universität München, Germany); Julian Habigt (Technische Universität München, Germany); Klaus Diepold (Technische Universität München, Germany) pp. 3325-3328

No-Reference Cross-Layer Video Quality Estimation Model Over Wireless Networks Yang Yan (BUPT, P.R. China)

pp. 3329-3332

No Reference Metric of Video Coding Quality Based on Parametric Analysis of Video Bitstream Osamu Sugimoto (KDDI R\&D Laboratories Inc., Japan) pp. 3333-3336

Audiovisual Quality Fusion Based on Relative Multimodal Complexity Junyong You (Norwegian University of Science and Technology, Norway); Jari Korhonen (Technical University of Denmark, Denmark); Ulrich Reiter (Norwegian University of Science and Technology (NTNU), Norway) pp. $3337-3340$

\section{WP.PB: Partial Differential Equations (Poster)}

An Improved Region-Based Model with Local Statistical Feature

Qi Ge (Nanjing Unverisity of Science \& Technology, P.R. China); Zhihui Wei (Nanjing University of Science and Technology, P.R. China)

pp. 3341-3344 
Active Contours with a Novel Distribution Metric for Complex Object Segmentation Shu-juan Peng (Huaqiao University, P.R. China); Xin Liu (Hong Kong Baptist University, P.R. China); Yiu-ming Cheung (Hong Kong Baptist University, Hong Kong) pp. $3345-3348$

\section{Texture Segmentation Based on Local Feature Histograms} Liyan Ma (Beijing Jiaotong University, P.R. China) pp. 3349-3352

Snake Based Unsupervised Texture Segmentation Using Gaussian Markov Random Field Models

Sasan Mahmoodi (University of Southampton, United Kingdom); Steve Gunn (, United Kingdom)

pp. 3353-3356

\section{Effective Image Noise Removal Based on Difference Eigenvalue}

Haiying Tian (The Sun Yat-Sen University, P.R. China); Hongmin Cai (The Sun YatSen University \& School of Information Science and Technology, P.R. China); Jianhuang Lai (Sun Yat-Sen University, P.R. China); X. Xu (Harvard Medical School, USA)

pp. 3357-3360

\section{Level Set Evolution with Locally Linear Classification for Image Segmentation} Wang Ying (NLPR, Institute of Automation, Chinese Academy of Sciences \& Institute of Automation, Chinese Academy of Sciences, P.R. China); Lingfeng Wang (National Laboratory of Pattern Recognition, P.R. China); Shiming Xiang (Institute of Automation, Chinese Academy of Sciences, P.R. China); Chunhong Pan (Institute of Automation, Chinese Academy of Sciences, P.R. China) pp. 3361-3364

\section{Cost Aggregation with Anisotropic Diffusion in Feature Space for Hybrid Stereo Matching}

Bumsub Ham (Yonsei University, Korea); Dongbo Min (Advanced Digital Sciences Center, Singapore); Kwang Hoon Sohn (Yonsei University, Korea) pp. 3365-3368

New Optimization Scheme for L2-Norm Total Variation Semi-Supervised Image Soft Labeling

Chia-Liang Tsai (National Taiwan University, Taiwan); Shao-Yi Chien (National Taiwan University, Taiwan) pp. 3369-3372

\section{Non-local Segmentation and Inpainting} Miyoun Jung (Ceremade, Universite Paris-Dauphine, France); Gabriel Peyré (CNRS and Université Paris-Dauphine, France); Laurent Cohen (CNRS and Université ParisDauphine, France) pp. 3373-3376

\section{PDEs Level Sets on Weighted Graphs}

Xavier Desquesnes (Université de Caen Basse-Normandie, France); Abderrahim Elmoataz (Université de Caen Basse-Normandi, France); Olivier Lezoray (Université de Caen Basse-Normandie \& Greyc UMR CNRS 6072, France) pp. $3377-3380$ 
Affine Morphological Shape Stable Boundary Regions (SSBR) for Image Representation

Petros Kapsalas (National Technical University of Athens, Greece); Stefanos Kollias (NTUA, Greece)

pp. 3381-3384

Discontinuous Seismic Horizon Tracking Based on a Poisson Equation with Incremental Dirichlet Boundary Conditions

Guillaume Zinck (University of Bordeaux, France); Marc Donias (IMS Laboratory University Bordeaux, France); Sébastien Guillon (TOTAL, France); Olivier Lavialle (Bordeaux 1 University - UMR IMS, France) pp. $3385-3388$

Reducing Aliasing in Images: a Simple Diffusion Equation Based on the Inverse Diffusivity

Djemel Ziou (Université de Sherbrooke, Canada); Alain Horé (Université de Sherbrooke, Canada) pp. 3389-3392

A New Deformable Model-Based Segmentation Approach for Accurate Extraction of the Kidney From Abdominal CT Images

Fahmi Khalifa (Bioimaging Laboratory \& University of Louisville, Louisville, KY, USA); Georgy Gimel'farb (University of Auckland, USA); Mohamed Abo El-Ghar (University of Mansoura, Egypt); Gulea Sokhadze (University of Louisville, USA); Rosemary Ouseph (University of Louisville, USA); Ayman Sabry El-Baz, PhD (University of Louisville, USA)

pp. 3393-3396

Level Set Segmentation with Robust Image Gradient Energy and Statistical Shape

Prior

Si Yong Yeo (School of Engineering, Swansea University, United Kingdom); Xianghua Xie (Swansea University, United Kingdom); Igor Sazonov (Swansea, United Kingdom); Perumal Nithiarasu (Swansea University, United Kingdom) pp. $3397-3400$

\section{WP.PC: Image Restoration and Enhancement (Poster)}

\section{Examplar-based Inpainting Based on Local Geometry}

Olivier Le Meur (University of Rennes 1, France); Josselin Gautier (University of Rennes 1, France); Christine Guillemot (INRIA, France) pp. 3401-3404

Wide-band Image Guided Visible-band Image Enhancement Youngjin Yoo (SAMSUNG Advanced Institute of Technology, Korea); Wonhee Choe (Samsung Electronics, Samsung Advanced Institute of Technology, Korea); SeongDeok Lee (Samsung Electronics, Samsung Advanced Institute of Technology, Korea) pp. $3405-3408$

Image Inpainting Via Weighted Sparse Non-Negative Matrix Factorization Yu-Xiong Wang (Tsinghua University, P.R. China); Yu-Jin Zhang (Tsinghua University, P.R. China) pp. 3409-3412 
Fragmented Aperture Imaging for Motion and Defocus Deblurring

Manuel Martinello (Heriot-Watt University, United Kingdom); Paulo Favaro (, United Kingdom)

pp. $3413-3416$

Image Enhancement Based on Retinex and Lightness Decomposition Wang Shuhang (Beihang University, P.R. China) pp. 3417-3420

Image Contrast Enhancement in Compressed Wavelet Domain Dongwook Cho (Concordia University, Canada); Tien D. Bui (Concordia University, Canada) pp. 3421-3424

\section{Color Histogram Diffusion for Image Enhancement} Taemin Kim (NASA Ames Research Center, USA) pp. $3425-3428$

Reconstruction of High Dynamic Range Images with Poisson Noise Modeling and Integrated Denoising

Bart Goossens (Ghent University, Belgium); Hiep Q Luong (Ghent University,

Belgium); Jan Aelterman (Ghent University, Belgium); Aleksandra Pižurica (Ghent University, Belgium); Wilfried Philips (Ghent University, Belgium) pp. 3429-3432

Gradient Domain Contrast Enhancement with Histogram-Guided Boundary Conditions Chulwoo Lee (Korea University, Korea); Chul Lee (Korea University, Korea); ChangSu Kim (Korea University, Korea) pp. 3433-3436

Noiseless No-Flash Photo Creation by Color Transform of Flash Image Keiichiro Shirai (Shinshu University, Japan) pp. 3437-3440

\section{Fast Image Inpainting Using Similarity of Subspace Method} Tomoki Hosoi (Yamatake Corporation, Japan); Koji Kobayashi (Yamatake Corpolation, Japan); Koichi Ito (Tohoku University, Japan); Takafumi Aoki (Tohoku University, Japan) pp. 3441-3444

\section{Automatic Foreground-Background Refocusing}

Alexander Loktyushin (Max Planck Institute for Biological Cybernetics, Germany); Stefan Harmeling (MPI for Biological Cybernetics, Germany) pp. 3445-3448

\section{Depth Map Super Resolution} Murat Gevrekci (ASELSAN, Turkey); Kubilay Pakin (Lead Design Engineer, Turkey) pp. 3449-3452

\section{Decoupled Inverse and Denoising for Image Deblurring: Variational BM3D-frame}

\section{Technique}

Vladimir Katkovnik, male (Tampre University of Technology, Finland); Aram Danielyan (Tampere University of Technology, Finland); Karen Egiazarian (Tampere University of Technology, Finland) pp. 3453-3456 
A Novel Iterative Image Restoration Algorithm Using Nonstationary Image Priors Esteban Vera (University of Arizona, USA); Mlguel Vega (University of Granada, Spain); Rafael Molina (Universidad de Granada, Spain); Aggelos K Katsaggelos (Northwestern University, USA) pp. 3457-3460

\section{Reconstructing Static Scene Viewed Through Smoke Using Video}

Ákos Kiss (Computer and Automation Research Institut, Hungary); Tamas Szirányi (Computer and Automation Research Institute of the Hungarian Academy of Sciences \& Pázmány Péter Catholic University, Hungary) pp. 3461-3464

\section{WP.PD: Scalable and Adaptive Methods for Video Coding (Poster)}

\section{A Novel Rate-Distortion Optimization Method of H.264/AVC Intra Coder}

Mohammed Golam Sarwer (Ryerson University, Canada); Jonathan Wu (University of Windsor, Canada); Xiao-Ping Zhang (Ryerson University, Canada) pp. 3465-3468

Intra-prediction with Adaptive Sub-sampling Yih Han Tan (Institute for Infocomm Research, Singapore); Chuohao Yeo (Institute for Infocomm Research, Singapore); Zhengguo Li (Institute for Inforcomm Research, Singapore); Susanto Rahardja (Institute for Infocomm Research, Singapore) pp. 3469-3472

\section{Theoretical Consideration of Global Motion Temporal Filtering} Andreas Krutz (Technische Universität Berlin, Germany); Alexander Glantz (Technische Universität Berlin, Germany); Thomas Sikora (Technische Universität Berlin, Germany) pp. 3473-3476

\section{Adaptive Loop Filter Technology Based on Analytical Design Considering Local} Image Characteristics

Tomonobu Yoshino (KDDI R\&D Laboratories Inc., Japan)

pp. 3477-3480

\section{An Efficient Key-Frame-Free Prediction Method for MGS of H.264/SVC}

Lili Zhao (Beihang University, P.R. China); You Zhou (Microsoft Research Asia, P.R. China); QinPing Zhao (Beihang University, P.R. China); Feng Wu (Microsoft Research Asia, P.R. China)

pp. 3481-3484

One Dimensional Prediction and Transform for Intra Coding

Changcai Lai (Research Dept., Huawei HiSilicon TechnologiesHuawei HiSilicon Technologies, P.R. China) pp. 3485-3488

Parallel Processing for Combined Intra Prediction in High Efficiency Video Coding Marta Mrak (BBC, United Kingdom); Andrea Gabriellini (BBC, United Kingdom); David Flynn (BBC, United Kingdom); Thomas Davies (BBC, United Kingdom) pp. 3489-3492 


\section{Extending SVC by Content-adaptive Spatial Scalability}

Yongzhe Wang (Shanghai Jiao Tong University, P.R. China); Nikolce Stefanoski (Disney Research Zurich, Switzerland); Manuel Lang (Disney Research, Zurich, Switzerland); Alexander Hornung (Disney Research Zurich, Switzerland); Aljoscha Smolic (Disney Research Zurich, Switzerland); Markus Gross (ETH Zurich, Switzerland) pp. 3493-3496

Rate Control Initialization Algorithm for Scalable Video Coding Sergio Sanz-Rodríguez (Universidad Carlos III de Madrid, Spain); Fernando Díaz-deMaría (Unversidad Carlos III de Madrid, Spain) pp. $3497-3500$

\section{Directional Adaptive Loop Filter for Video Coding} Yunfei Zheng (Qualcomm, USA); Peng Yin (Dolby Laboratories, Inc., USA); Qian Xu (Intel Inc, USA); Joel Sole (Qualcomm, USA); Xiaoan Lu (Thomson, Inc., USA) pp. 3501-3504

\section{Improved for/sor-Based Video Coding and Its Performance Analysis} Jewon Kang (University of Southern California, USA); Chung-Cheng Lou (University of Southern California, USA); Seung-hwan Kim (University of Southern California, USA); C.-C. Jay Kuo (University of Southern California, USA) pp. 3505-3508

Video Encoder Based on Lifting Transforms on Graphs Eduardo Martínez-Enríquez (Universidad Carlos III de Madrid, Spain); Fernando Díaz-de-María (Unversidad Carlos III de Madrid, Spain); Antonio Ortega (USC, USA) pp. 3509-3512

\section{A Denoising Approach for Iterative Side Information Creation in Distributed Video Coding} Joao Ascenso (ISEL \& IT, Portugal); Catarina Brites (IST - IT, Portugal); Fernando Pereira (IST-IT, Portugal) pp. 3513-3516

\section{Periodic Entropy Coder Initialization for Wavefront Decoding of Video Bitstream} Kiran M. Misra (Sharp Laboratories of America Inc., USA); Jie Zhao (Sharp Labs of America, USA); Andrew Segall (Sharp Labs, USA) pp. $3517-3520$

McFis in Hierarchical Bipredictve Pictures-Based Video Coding for Referencing the Stable Area in a Scene Manoranjan Paul (Charles Sturt University, Australia); Weisi Lin (Nanyang Technological University, Singapore); C. T. Lau (Nanyang Technological University, Singapore); Bu Sung Lee (Nanyang Technological University, Singapore) pp. 3521-3524

\section{WP.PE: Electronic Imagery: Detection, Classification and Restoration (Poster)}

\section{Image Categorization Through Optimum Path Forest and Visual Words} João Paulo Papa (UNESP - Univ Estadual Paulista, Brazil); Anderson Rocha (State University of Campinas, Brazil) 
pp. $3525-3528$

Automatic Segmentation for Arabic Characters in Handwriting Documents Ahmed Lawgali (Northumbria University, United Kingdom); Ahmed Bouridane (Northumbria University, United Kingdom); Maia Angelova (Northumbria University, United Kingdom) pp. 3529-3532

Point Object Detection Using a NL-means Type Filter Laure Genin (ONERA \& EADS Astrium, France); Frédéric Champagnat (ONERA, France); Guy Le Besnerais (ONERA, France); Laurent Coret (EADS-Astrium, France) pp. 3533-3536

\section{Removing the Artifacts From Artwork Cross-Section Images}

Miroslav Beneš (Institute of Information Theory and Automation, Czech Republic); Barbara Zitová (Institute of Information Theory and Automation, Czech Republic); Jan Blažek (Institute of Information Theory and Automation \& Charles University, Czech Republic); Janka Hradilová (Academic Laboratory of Materials Research of Paintings, Czech Republic); David Hradil (Institute of Inorganic Chemistry, Czech Republic) pp. 3537-3540

Hierarchical Hybrid MLP/HMM or Rather MLP Features for a Discriminatively Trained Gaussian HMM: A Comparison for Offline Handwriting Recognition

Philippe Dreuw (RWTH Aachen University, Germany); Patrick Doetsch (RWTH Aachen University, Germany); Christian Plahl (RWTH Aachen University, Germany); Hermann Ney (RWTH Aachen, Germany) pp. 3541-3544

\section{Computerized Paleography: Tools for Historical Manuscripts}

Lior Wolf (Tel-Aviv University, Israel); Liza Potikha (Tel-Aviv University, Israel); Nahcum Dershowitz (Tel-Aviv University, Israel); Roni Shweka (Genazim, Israel); Yaacov Choueka (Gwenzaim, Israel) pp. $3545-3548$

\section{Edge Noise Removal in Bilevel Graphical Document Images Using Sparse}

\section{Representation}

Thai V. Hoang (LORIA, Université Nancy 2, France); Elisa Barney Smith (Boise State University, USA); Salvatore Tabbone (University Nancy 2, France) pp. 3549-3552

\section{Fast Detection of Small Infrared Objects in Maritime Scenes Using Local Minimum} Patterns

Baojun Qi (National University of Defense Technology, P.R. China); Tao Wu (National University of Defense Technology, P.R. China); Bin Dai (National University of Defense Technology, P.R. China); Hangen He (National University of Defense Technology, P.R. China) pp. 3553-3556

\section{Face Liveness Detection Under Bad Illumination Conditions}

Bruno Peixoto (University of Campinas (Unicamp), Brazil); Carolina Michelassi (University of Campinas (Unicamp), Brazil); Anderson Rocha (State University of Campinas, Brazil) pp. $3557-3560$ 


\section{WP.PF: Object Detection and Recognition (Poster)}

\section{Beyond Straight Lines - Object Detection Using Curvature}

Antonio Monroy (IWR - University of Heidelberg, Germany); Angela Eigenstetter (IWR - University of Heidelberg, Germany); Björn Ommer (IWR - University of Heidelberg, Germany) pp. 3561-3564

Partial Least Squares Based Subwindow Search for Pedestrian Detection Jinchen Wu (National Laboratory of Pattern Recognition, Institute of Automation, Chinese Academy of Sciences, P.R. China); Wei Chen (Institute of Automation, Chinese Academy of Sciences, P.R. China); Kaiqi Huang (Chinese Academy of Sciences, P.R. China); Tieniu Tan (NLPR, P.R. China) pp. 3565-3568

\section{Boosting Based Object Detection Using a Geometric Model} Katharina Quast (Friedrich-Alexander University Erlangen-Nuremberg, Germany); Christoph Seeger (University of Erlangen-Nuremberg, Germany); Mohan Trivedi (UCSD, USA); Andre Kaup (University of Erlangen-Nuremberg, Germany) pp. 3569-3572

\section{Nonlinear L1-norm Minimization Learning for Human Detection}

Ran Xu (Graduate University of Chinese Academy of Sciences, P.R. China); Jianbin Jiao (Graduate University of Chinese Academy of Sciences, P.R. China); Qixiang Ye (Graduate University of Chinese Academy of Sciences, P.R. China)

pp. 3573-3576

\section{Automatic Nesting Seabird Detection Based on Boosted HOG-LBP Descriptors}

Chunmei Qing (University of Lincoln, United Kingdom); Patrick Dickinson (University of Lincoln, United Kingdom); Shaun Lawson (University of Lincoln, United Kingdom); Robin Freeman (Microsoft Research, United Kingdom) pp. 3577-3580

\section{Hollow TV Logo Detection}

Liang Zhang (Institute of Computing Technology, Chinese Academy of Sciences, P.R. China); Tian Xia (Institute of Computing Technology, CAS, P.R. China); Yongdong Zhang (Institute of Computing Technology, Chinese Academy of Sciences, P.R. China); Jintao Li (Institute of Computing Technology, Chinese Academy of Sciences, P.R. China) pp. 3581-3584

\section{Pose Estimation by Local Procrustes Regression}

Bisser Raytchev (Hiroshima University, Japan); Kazuya Terakado (Hiroshima University, Japan); Toru Tamaki (Hiroshima University, Japan); Kazufumi Kaneda (Hiroshima University, Japan) pp. 3585-3588

\section{Fast Human Detection Using Node-Combined Part Detector}

Song Cao (Tsinghua University, P.R. China); Genquan Duan (Tsinghua University, P.R. China); Haizhou Ai (Tsinghua University, P.R. China) pp. 3589-3592 
Real-time Detection Via Homography Mapping of Foreground Polygons From Multiple Cameras

Ming Xu (Xi'an Jiaotong-Liverpool University, P.R. China)

pp. 3593-3596

Robust Abandoned Object Detection Using Region-Level Analysis Jiyan Pan (Carnegie Mellon University, USA); Quanfu Fan (IBM T. J. Watson Research, USA); Sharath Pankanti (IBM Research, USA) pp. $3597-3600$

\section{Canonical Correlation Analysis of Local Feature Set for View-based Object}

\section{Recognition}

Xian-Hua Han (Ritsumeikan University, Japan); Yen-Wei Chen (Ritsumeikan University, Japan); Ruan (OMRON Corp., Japan) pp. 3601-3604

Hand Shape Recognition Using Distance Transform and Shape Decomposition Junyeong Choi (Hanyang University, Korea); Hanhoon Park (NHK Science \& Technology Research Laboratories, Japan); Jong-II Park (Hanyang University, Korea) pp. $3605-3608$

\section{Human Detection with Contour-based Local Motion Binary Patterns} Thanh Duc Nguyen (University of Wollongong, Australia); Philip Ogunbona (University of Wollongong, Australia); Wanqing Li (University of Wollongong, Australia) pp. 3609-3612

Pictorial Structures for Object Recognition and Part Labeling in Drawings Amir Sadovnik (Cornell University, USA); Tsuhan Chen (Cornell University, USA) pp. 3613-3616

Robust Head Pose Estimation Via Convex Regularized Sparse Regression Ji Hao (Carnegie Mellon University, USA); Risheng Liu (Dalian University of Technology, P.R. China); Fei Su (Beijing university of posts and telecommunications, P.R. China); Zhixun Su (Dalian University of Technology, P.R. China); Yan Tian (Hikvision Digital Technology Co. Ltd, P.R. China) pp. $3617-3620$

\section{WP.PG: Video Retrieval (Poster)}

\section{Towards a Better Understanding of Model-Free Semantic Concept Detection for} Annotation and Near-Duplicate Video Clip Detection

Hyun-seok Min (KAIST, Korea); JaeYoung Choi (Korea Advanced Institute of Science and Technology (KAIST), Korea); Wesley Marcel De Neve (Korea Advanced Institute of Science and Technology (KAIST), Korea); Yong Man Ro (KAIST, Korea) pp. 3621-3624

\section{Quadtree Classified Vector Quantization Based Image Retrieval Scheme} Hsin-Hui Chen (National Taiwan University, Taiwan); Hsin-Teng Sheu (Tung-Nan University, Taiwan); Jian-Jiun Ding (National Taiwan University, Taiwan) pp. 3625-3628 
A Multimodal Video Copy Detection Approach with Sequential Pyramid Matching Yonghong Tian (National Engineering Lab for Video Technology, Peking University, P.R. China); Menglin Jiang (Peking University, P.R. China); Luntian Mou (Chinese Academy of Sciences, P.R. China); Xiaoyu Fang (Peking University, P.R. China); Tiejun Huang (Peking University, P.R. China) pp. 3629-3632

Retrieving Video Shots in Semantic Brain Imaging Space Using Manifold-Ranking Xiang Ji (Northwestern Polytechnical University, P.R. China); Junwei Han (Northwestern Polytechnical University, P.R. China); Xintao Hu (Northwestern Polytechnical University, P.R. China); Kaiming Li (Northwestern Polytechnical University, P.R. China); Fan Deng (University of Georgia, USA); Jun Fang (Northwestern Polytechnical University, P.R. China); Guo Lei (Northwestern Polytechnical University, P.R. China); Tianming Liu (University of Georgia, USA) pp. 3633-3636

\section{Multivariate Texture Retrieval Using the Geodesic Distance Between Elliptically Distributed Random Variable}

Lionel Bombrun (IMS Laboratory - University Bordeaux \& Groupe Signal, France); Yannick Berthoumieu (IMS Laboratory - University Bordeaux, France); Nour-Eddine Lasmar (IMS Laboratory - University Bordeaux, France); Geert Verdoolaege (Ghent University, Belgium)

pp. 3637-3640

\section{Action Scene Detection From Motion and Events}

Robert Sorschag (Vienna University of Technology, Austria); Markus Hörhan (Vienna University of Technology, Austria)

pp. 3641-3644

Multi-dimensional Evolutionary Feature Synthesis for Content-based Image Retrieval Serkan Kiranyaz (Tampere University of Technology, Finland); Jenni Pulkkinen (Tampere University of Technology, Finland); Turker Ince (, Turkey); Moncef Gabbouj (Tampere University of Technology \& Tampere, Finland, Finland) pp. 3645-3648

Integrating Distance Metric Learning Into Label Propagation Model for Multi-label Image Annotation

Bin Wang (Shanghai Jiao Tong University, P.R. China); Yi Shen (Shanghai Jiao Tong University, P.R. China); Yuncai Liu (Shanghai Jiaotong University, P.R. China) pp. 3649-3652

\section{Using Context Saliency for Movie Shot Classification}

Min Xu (University of Technology Sydney, Australia); Jinqiao Wang (Institute of Automation (IA) Chinese Academy of Sciences (CAS), P.R. China); Muhammad Abul Hasan (University of Technology Sydney, Australia); Xiangjian He (University of Technology, Sydney, Australia); Changsheng Xu (Institute of Automation, Chinese Academy of Sciences \& China-Singapore Institute of Digial Media, P.R. China); Hanqing Lu (the Institute of Automation, Chinese Academy of Sciences, P.R. China); Jesse $S$ Jin (University of Newcastle, Australia)

pp. 3653-3656 
View-based 3D Model Retrieval Using Two-Level Spatial Structure

Pengjie Li (Beijing University of Posts and Telecommunications, P.R. China); Huadong Ma (Beijing University of Posts and Telecommunications, P.R. China); AnLong Ming (Beijing University of Posts and Telecommunications, P.R. China) pp. $3657-3660$

\section{A Bag-of-Regions Approach to Sketch-Based Image Retrieval}

Rui Hu (University of Surrey, United Kingdom); Tinghuai Wang (University of Surrey, United Kingdom); John Philip Collomosse (University of Surrey, United Kingdom) pp. 3661-3664

Image Coding with Face Descriptors Embedding

Alberto Boschetti (University of Brescia, Italy); Nicola Adami (University of Brescia, Italy); Riccardo Leonardi (University of Brescia, Italy); Masahiro Okuda (The University of Kitakyushu, Japan)

pp. 3665-3668

On the Surprisingly Accurate Transfer of Image Parameters Between Medical and Solar Images Juan Banda (Montana State University, USA); Rafal Angryk (Montana State University, USA); Petrus C Martens (Montana State University \& Harvard Smithsonian Center for Astrophysics, USA)

pp. 3669-3672

\section{Comparison of Video Sequences with Histograms of Motion Patterns} Jurandy Almeida (University of Campinas, Brazil); Neucimar Leite (State University of Campinas, Brazil); Ricardo da Silva Torres (Institute of Computing, State University of Campinas, Brazil)

pp. 3673-3676

\section{Location-Based Image Retrieval for Urban Environments}

Jerry Zhang (University of California, Berkeley, USA); Aaron Hallquist (University of California, Berkeley, USA); Avideh Zakhor (University of California at Berkeley, USA) pp. 3677-3680

\section{WP.L5: Advances in Transforms for Video Coding (Special Session)}

\section{One-Dimensional Directional Unified Transform for Intra Coding}

Jun Yamaguchi (Toshiba, Japan); Taichiro Shiodera (Toshiba Corporation, Japan); Saori Asaka (Toshiba Corporation, Japan); Akiyuki Tanizawa (Toshiba Corporation, Japan); Tomoo Yamakage (Toshiba Corporation, Japan) pp. $3681-3684$

\section{Low-complexity Mode-Dependent KLT for Block-Based Intra Coding}

Chuohao Yeo (Institute for Infocomm Research, Singapore); Yih Han Tan (Institute for Infocomm Research, Singapore); Zhengguo Li (Institute for Inforcomm Research, Singapore)

pp. 3685-3688

break 


\section{Rotational Transform for Image and Video Compression}

Elena Alshina (Samsung Electronics \& DMC R\&D Center, Korea); Alexander Alshin (Samsung Electronics Co., Ltd, Korea); Felix Fernandes (Samsung Electronics Co., Ltd, USA)

pp. 3689-3692

\section{Transform Coding in the HEVC Test Model}

Martin Winken (Fraunhofer HHI, Germany); Philipp Helle (Fraunhofer HHI, Germany);

Detlev Marpe (Fraunhofer Institute for Telecommunications - Heinrich Hertz Institute,

Germany); Heiko Schwarz (Fraunhofer HHI, Germany); Thomas Wiegand

(Fraunhofer Institute for Telecommunications - Heinrich-Hertz-Institute, Germany) pp. 3693-3696

\section{Design of Non-Separable Transforms for Directional 2-D Sources}

Bing Zeng (Hong Kong University of Science and Technology, Hong Kong) pp. $3697-3700$

\section{Distortion Estimates for Adaptive Temporal Decompositions of Video Under}

Displacement Errors and Quantization Noise

Fabio Verdicchio (University of Aberdeen, United Kingdom); Yiannis Andreopoulos

(University College London, United Kingdom)

pp. 3701-3704

Inter Prediction Using Lapped Transforms for Advanced Video Coding

Rafael Galvão de Oliveira (Télécom PArisTech, France); Beatrice Pesquet (Telecom

Paristech, France); Maria Trocan (I. S. E. P., France)

pp. 3705-3708 
On behalf of the ICIP 2011 Organizing Committee, we are warmly welcoming you to Brussels, the capital of the Kingdom of Belgium and the home to the European headquarters, a buzzing, influential city where history is made. The city is a key European commercial hub, a base for many major international companies. This cosmopolitan city that loves good food lives life its way and expresses itself in a style very much its own: sometimes rebellious and mischievous, sometimes thoughtful and composed, but always very likeable. Despite its European dimension and despite all the different languages spoken on the corner of every street, Brussels is still inspired by a very "village-like" spirit. Of course, it's well known for its GrandPlace, its Atomium, its Manneken-Pis, its Gueuze and its Kriek, its waffles and its chocolates... (don't miss them!). The Brussels-Capital region consists of 19 communes and covers a total surface area of 16,179 ha. The population has around 990,000 inhabitants. Roughly a quarter of the city's total population is foreign. Brussels has a temperate, maritime climate. The average temperature in September is around $16^{\circ}$ Celsius $\left( \pm 60^{\circ}\right.$ Fahrenheit).

Brussels and Belgium, being at the crossroad of France, Germany and United Kingdom has been the place where Europe has been shaped. It is crossed by the border which separates Latin Europe from German Europe, which is the place where in 57 BC Julius Caesar stopped its first conquest of the Gaul. This line still separates today the two main communities of Belgium, the Flemish and the Walloon. Belgium nourishes diversity, creativity, art and technology. The Flemish textile industry came from the middle age, while the steel and glass industry made Belgium one of the main industrial countries at the beginning of the 20th century. These industrial golden ages of Belgium gave rise to art and science creativity, among which one can cite the Flemish school of Painting in the 15th century or the Solvay congresses in the beginning of the 20th century where all the key Physicists of the World, including Albert Einstein and Marie Curie, were shaping the modern Physics in Brussels under the sponsorship of Ernest Solvay. We are very proud to welcome ICIP in the continuity of this long tradition and spirit of modernity.

ICIP is the premier forum for the presentation of technological advances and research results in the fields of theoretical, experimental, and applied image and video processing. ICIP 2011, the 18th in the series that has been held annually since 1994, brings together leading engineers and scientists in image processing from around the world. As its previous editions ICIP 2011 targets an excellence level by selecting outstanding papers with the aid of an elaborate panel of experts that evaluated the papers. The Technical Program Chairs, Special Session Chairs and Tutorial Chairs supported by many collaborators managed this process while aiming at an acceptance rate of $40 \%$. The three main 2011 themes, human computer interfacing, astronomy and cultural heritage, are reflected in the plenary talks and multiple lecture and poster sessions. This conference also awards best student papers and best papers via a meticulous process involving expert reviewers.

The conference center, the Square, is housed in the centre of Brussels in the extensive former Palais des Congrés, an elegant, architecturally significant building originally constructed for the 1958 World Expo. Many of the original features, including expansive murals by Paul Delvaux, René Magritte and Louis van Lint, have been carefully restored and are now juxtaposed with contemporary design conceived by a team of leading European designers. With its spectacular views over the Brussels skyline, Square is somewhere to inspire the mind to greater heights and ambition. Brussels is the thriving centre of Europe, and Square is right in the heart of it, just a short stroll from all of the historic city's major attractions.

The welcome reception takes place in the proximity of the square in the King's Gallery (Galerie du Roi), which is one of three galleries that are better known as the Saint-Hubertus Royal Gallery. The galleries housed some 70 luxury boutiques (54 today) and about 100 private flats, all of which have been rented out since 1850. The banquet is hosted by AutoWorld, one of the most important and complete exhibitions of automobiles in Europe with more than 350 vehicles offering an overview of the general history of the car industry since its very begins.

Organizing a conference like ICIP always involves many persons and addressing them individually in this word of thank would not be feasible, but we do want to mention a few people that played a key role in organizing this event. We would like to thank especially the Technical Program Chairs, Prof. Inald Lagendijk 
and Prof. Pierre Moulin for their guidance and hard work putting together this year's technical program, and the Local Arrangement Chairs, Alasdair Grant and Dr.

Véronique Delouille, for managing the event's overall complexity and its smooth organization. They were assisted by Destrée Organisation as the Professional Congress Organizer (PCO) and by Carlson Wagonlit Travel to accommodate the social events and hotel reservations. Finally, we would like to express our gratitude to the IEEE staff and in particular to Lisa Schwarzbek for guiding us through this adventurous experience.

Evidently, we need also to thank our sponsors: IEEE and the IEEE Signal Processing Society, and our supporters: the Funds for Scientific Research in Flanders and Wallonie (FWO and FNRS), the Belgian Science Policy (BELSPO) project BCRYPT, INRIA, Hewlett-Packard, Huawei, Barco, Wiley-Blackwell, VisitBrussels and Duvel Moortgat.

It is an honor to host ICIP 2011 and we hope that attendees will have exciting and fruitful experience paving the way for future research and developments in the domain. We welcome you to Brussels and hope you will have a joyful stay!

Bienvenue à Bruxelles, Welkom in Brussel, Willkommen in Brüssel, Welcome to Brussels!

Prof. Benoit Macq, General Chair, ICIP 2011

Prof. Peter Schelkens, General Co-Chair, ICIP 2011 
It is our pleasure to welcome you to the 2011 18th IEEE International Conference on Image Processing in Brussels, the world capital of beer and chocolate! Hopefully attending ICIP 2011 will stimulate both your palate as your intellect and will be an exciting, fruitful, and inspiring experience.

This year we have received 2245 paper submissions from 67 countries spread all over the globe. The Asia/ Pacific region provided $44.4 \%$ of the authors, Europe, the Middle East and Africa $37.0 \%$, North America $15.6 \%$, and Latin America 3.0\%.

The organizing committee had all submitted papers subjected to peer evaluation by 1113 volunteers who were selected based on their expertise area. They provided over 7,000 reviews, i.e., an average of about 3.2 per paper. The review process was managed per EDICS domain by 61 Area Chairs who are members of the Image, Video, and Multidimensional Signal Processing (IVMSP) Technical Committee of the IEEE Signal Processing Society, members of the Multimedia Signal Processing (MMSP) Technical Committee, or otherwise leading researchers within the IEEE Signal Processing Society Image Processing community. The Area Chairs monitored the review process and made recommendations for each paper. A total of 889 regular papers (40.6\%) have been accepted and are presented in 48 lectures and 42 poster sessions. We would like to sincerely thank everybody involved in the review process: your individual contributions have shaped the ICIP 2011 program.

The ICIP 2011 technical program includes three plenary lectures addressing the main themes of the conference: human computer interfacing, cultural heritage and astronomy. We are therefore delighted to announce the following plenary lectures at this year's ICIP conference:

- "Seeing and the Brain", by Prof. Brian Wandell from Stanford University, USA.

- "Sparsity and Astronomical Data Analysis", by Dr. Jean-Luc Starck from the Commission for Atomic Energy (CEA), France.

- "Distinguishing the 'Hand' of the Master?," by Prof. Ingrid Daubechies from Duke University, USA.

The conference kicks off on Sunday, 11th September, with 5 tutorials (3 in the morning and 2 in the afternoon). These tutorials were selected from 17 proposals submitted in response to the Call for Tutorials by a team of experts under the guidance of Prof. Jean-Philippe Thiran and Dr. Ton Kalker. The evaluation process resulted in the selection of the following state-of-the-art tutorials on vibrant research topics in image processing:

- "Convex Optimization Methods for Image Processing" by Xavier Bresson and Thomas Pock.

- "Image Analysis in Very High Resolution Optical Remote Sensing" by Jocelyn Chanussot.

- "Image and Video Analysis using Local Binary Pattern Variants" by Matti Pietikäinen and Janne Heikkilä.

- "Spatio-temporal Filtering for Multi-object Tracking in Image Sequences" by Andrea Cavallaro and Emilio Maggio.

- "ToF (Time of Flight) Depth Sensor Image Processing: from Principle to Applications" by Seungkyu Lee, Albert J.P. Theuwissen, Radu Horaud, Miles Hansard, Hwasup Lim and SeongJin Kim.

The ICIP 2011 program also offers 8 special sessions covering key research topics. The special sessions were selected after a meticulous reviews process of 12 submitted special session proposals in response to the Call for Special Sessions under the guidance of the Special Session Chairs, Prof. Edward Delp and Prof. Ferran Marques. Each paper in the special sessions was reviewed by, at least, four reviewers. The ICIP 2011 special sessions and corresponding organizers are:

- "Advances in Motion Representation for Video Coding", by Thomas Sikora, and Andreas Krutz.

- "Advances in Transforms for Video Coding", by Marta Mrak, and Robert Cohen. 
- "Analysis of Microscopy and Reconstructive Images for Applications in Medicine and Biology", by Laure Blanc-Feraud, Dave Burton, Aymeric Histace, Bogdan Matuszewski, Chris Moore, and Frederic Precioso.

- "Astronomy and Cosmology", by Yves Wiaux.

- "Compression of High-Dimensional Media Data for Interactive Navigation", by Gene Cheung, Pier Luigi Dragotti, and Vladan Velisavljevic.

- "Distributed Compression: Multimedia Applications", by Vladimir Stankovic, Lina Stankovic, and Samuel Cheng.

- "Modern Shape from Shading and Beyond", by Michael Breuß, Ariel Tankus, and Oliver Vogel.

- "Recent Advances in Web-scale Image Annotation", by Clement Chia Nanyang, Yiqun Hu, and Jinjun Wang.

To recognize top research results, ICIP 2011 includes two types of awards:

- Best Paper Award, supported by Hewlett-Packard. After nomination by the Area Chairs and guided by the reviewers' scores and comments, the nominated papers have been reevaluated by an independent panel of experts.

- Best Student Paper Awards, supported by Huawei, Barco and Wiley-Blackwell. For these awards the first author needs to be a student upon submission of the paper. ICIP 2011 has followed the process initiated at last year's ICIP in Hong Kong, highlighting the importance of the paper presentation. Eight Best Student Paper Award candidates have been selected through a review process from a list of 50 top scoring papers nominated by senior researchers.

The eight nominated papers are invited to present their work in a Best Student Paper Award Special Session. A jury composed by the Awards Chairs will score the candidates by considering all relevant reviewing criteria, including the quality of the oral presentation.

The award process was managed by the Awards Chairs, Prof. Pascal Frossard and Prof. Béatrice Pesquet-Popescu. They will present the awards during the conference banquet.

As Technical Program Chairs, we would like to thank the plenary speakers, tutorials presenters, reviewers, session chairs, and all authors and presenters for their contributions to ICIP 2011. In particular we would like to thank the Area Chairs for their instrumental support for building a high quality ICIP 2011 program. Finally, we would like to express our appreciation for the Destrée Organisation, in particular Nicolas Le Brun and his team, for their support in organizing ICIP 2011.

We look forward to welcoming you in Brussels,

Prof. Inald Lagendijk and Prof. Pierre Moulin ICIP 2011 Technical Program Chairs 


\section{IEEE ICIP2011: 2011 18th IEEE International Conference on Image Processing}

$\mathrm{IC}$ $\mathrm{P}$

20112011 18th IEEE International Conference on Image Processing took BRUSSELS place 11-14 September 2011 in Brussels, Belgium.

\section{IEEE catalog number: CFP11CIP-ART \\ ISBN: $\quad 978-1-4577-1303-3$}

Copyright and Reprint Permission: Abstracting is permitted with credit to the source. Libraries are permitted to photocopy beyond the limit of U.S. copyright law for private use of patrons those articles in this volume that carry a code at the bottom of the first page, provided the per-copy fee indicated in the code is paid through Copyright Clearance Center, 222 Rosewood Drive, Danvers, MA 01923. For other copying, reprint or republication permission, write to IEEE Copyrights Manager, IEEE Operations Center, 445 Hoes Lane, Piscataway, NJ 08854. All rights reserved. Copyright $\odot 2011$ by IEEE.
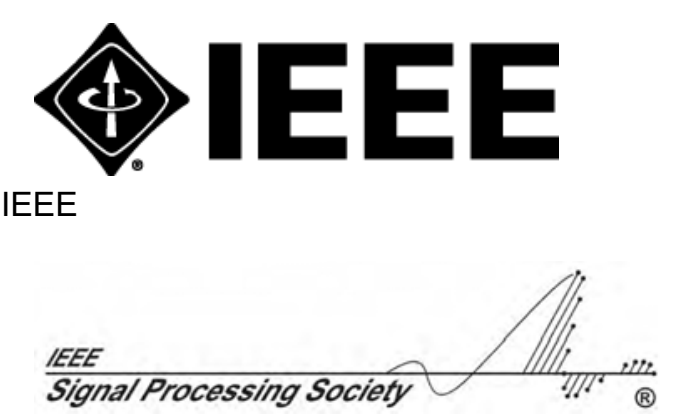

IEEE Signal Processing Society 